\title{
Localisation and function of Slam in the early Drosophila embryo
}

\author{
Dissertation \\ for the award of the degree \\ "Doctor rerum naturalium (Dr.rer.nat.)" \\ in the GGNB program "Genes and Development" \\ at the Georg-August-Universität Göttingen \\ Faculty of Biology
}

submitted by

Sreemukta Acharya

born in Akividu, India

Göttingen, August 2014 


\section{MEMBERS OF THE THESIS COMMITTEE}

Prof. Jörg Großhans (Supervisor, reviewer)

Department of Developmental Biochemistry, University of Göttingen

\section{Prof. Reinhard Schuh (Reviewer)}

Department of Molecular Developmental Biology, Max Planck Institute for Biophysical Chemistry

\section{Prof. Henning Urlaub}

Bioanalytical Mass Spectometry Group, Max Planck Institute for Biophysical Chemistry

Date of oral examination: 20.10 .2014 


\section{AFFIDAVIT}

I hereby declare that I prepared the PhD thesis "Localisation and function of Slam in the early Drosophila embryo" on my own with no other sources and aids than quoted.

Sreemukta Acharya,

Göttingen, 29.08.2014 


\section{LIST OF PUBLICATIONS}

Acharya, S.*, Laupsien, P.*, WenzI, C., Yan, S., and Großhans, J. (2014). Function and dynamics of slam in furrow formation in early Drosophila embryo. Dev. Biol. 386, 371-384.

*These authors contributed equally. 


\section{TABLE OF CONTENTS}

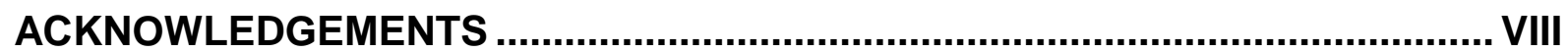

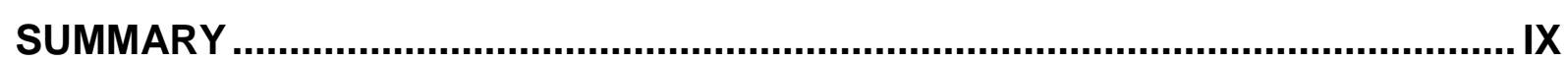

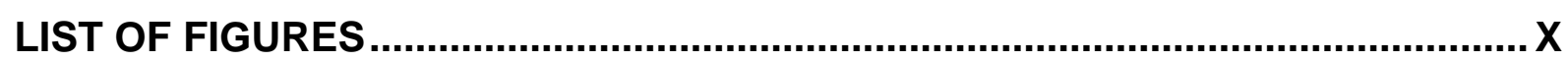

LIST OF TABLES

LIST OF MOVIES.......................................................................................... X

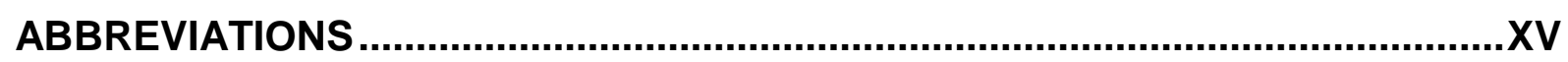

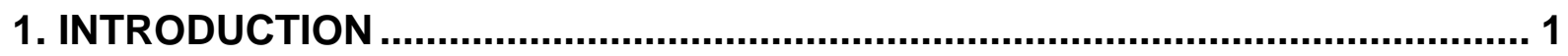

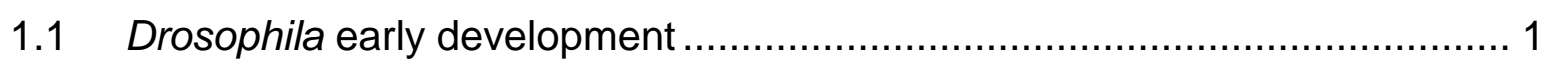

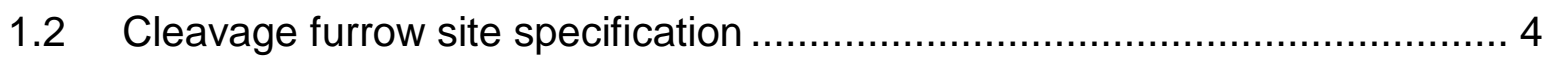

1.3 Initiation and maintenance of membrane invagination.................................... 6

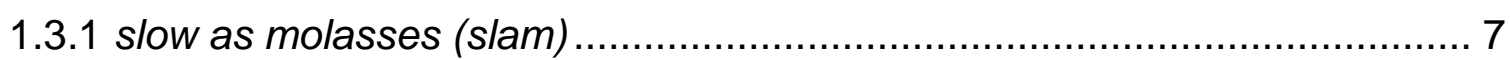

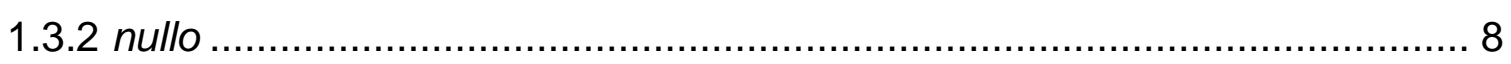

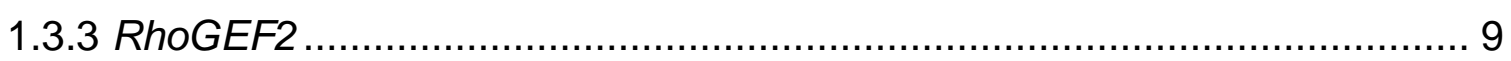

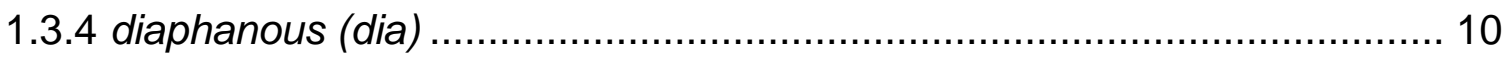

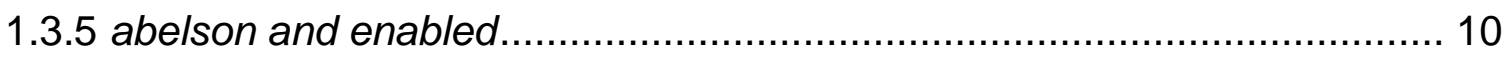

1.3.6 Cytoskeletal components .................................................................. 11

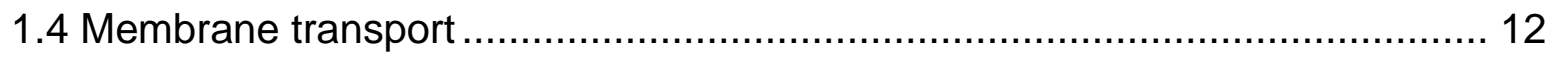

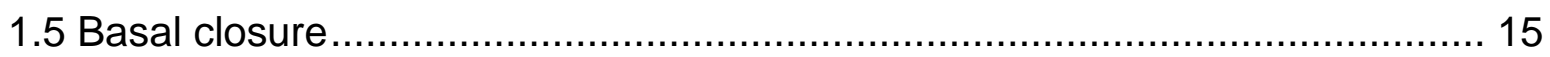

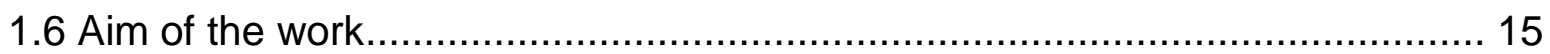

2. MATERIALS AND METHODS

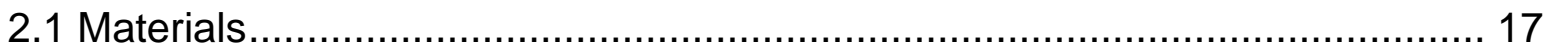

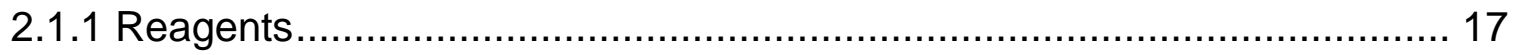

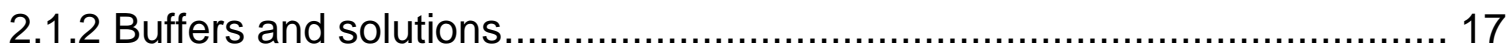

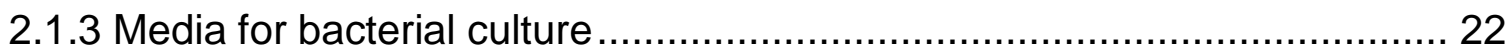

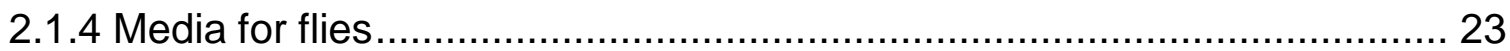

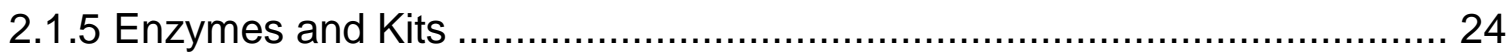

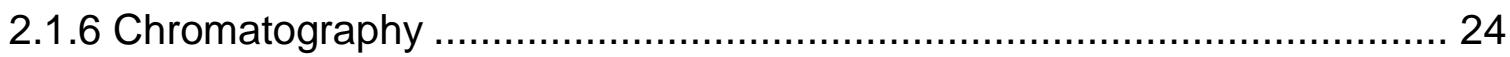

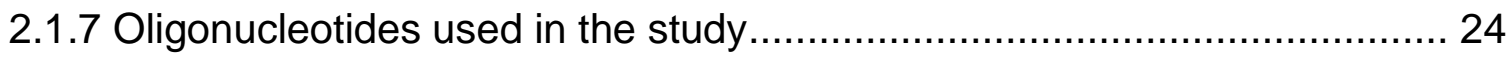

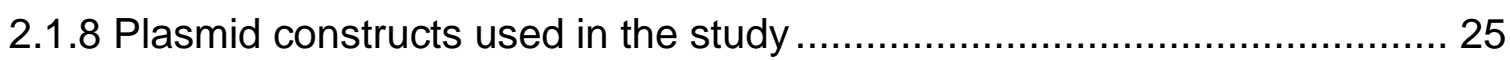

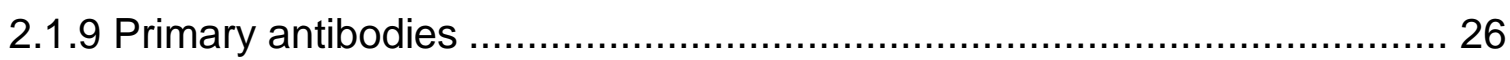

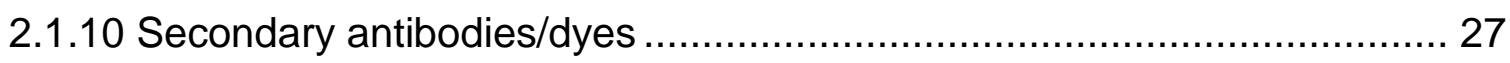

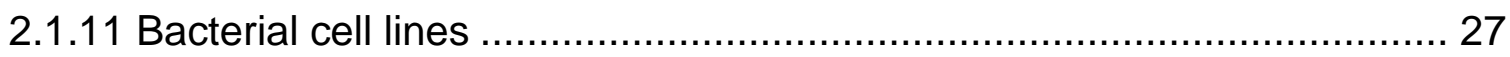

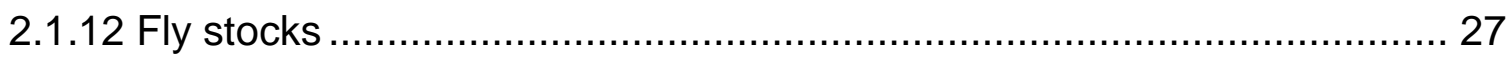

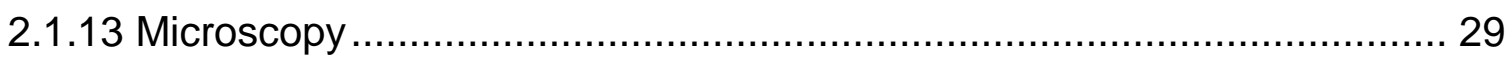




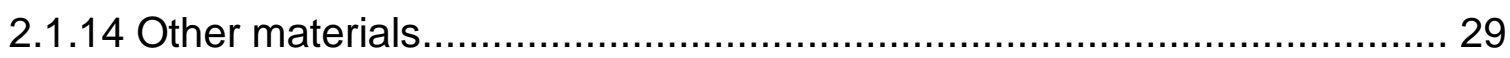

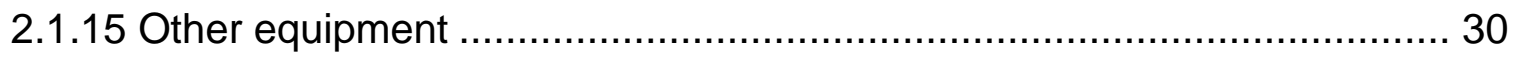

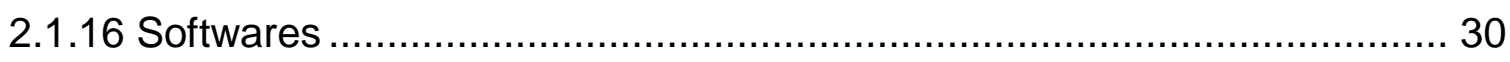

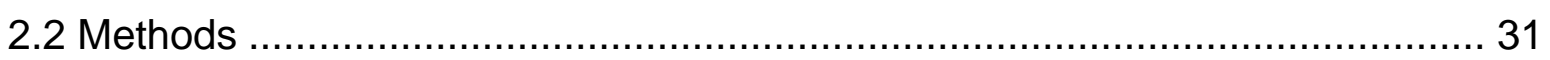

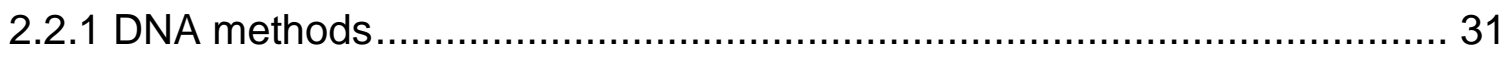

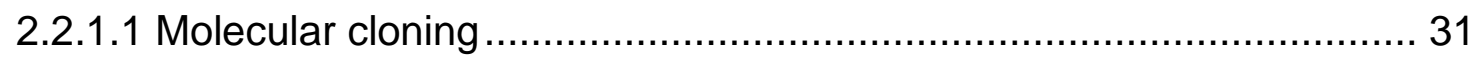

2.2.1.2 Polymerase chain reaction (PCR) .............................................. 31

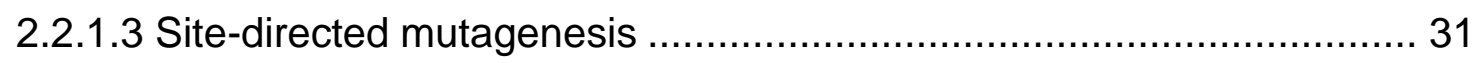

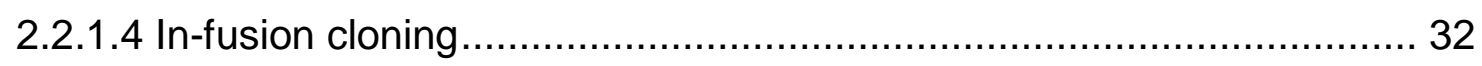

2.2.1.5 Purification of DNA/RNA by phenol-chloroform extraction ................. 32

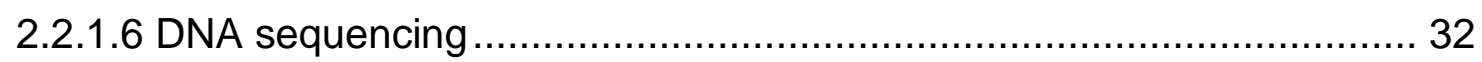

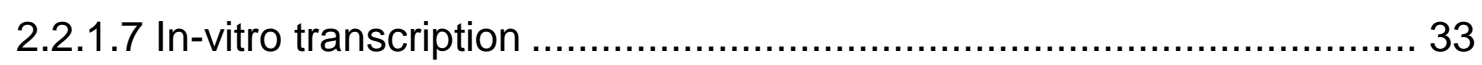

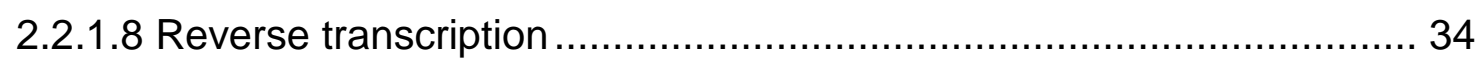

2.2.1.9 Quantitative real-time PCR (qRT-PCR) ........................................ 34

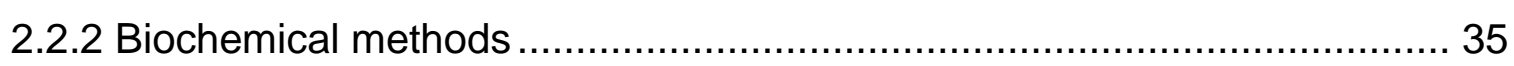

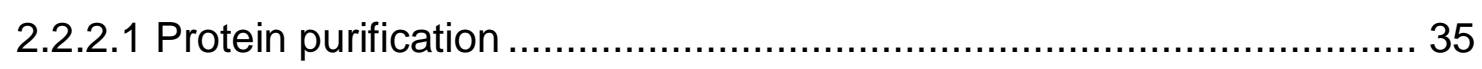

2.2.2.2 Affinity purification of antibodies ................................................ 36

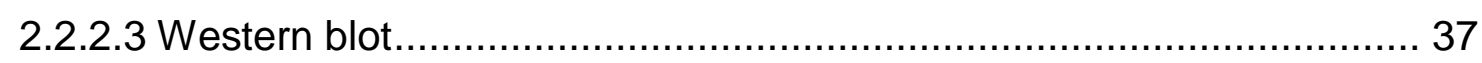

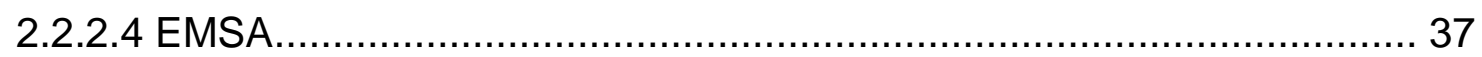

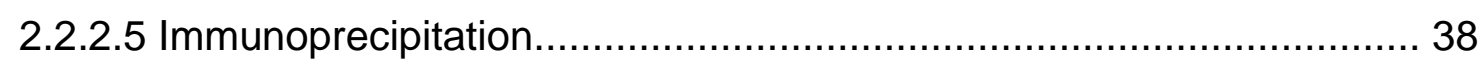

2.2.2.6 Pulldown of GFPslam using paramagnetic Streptavidin beads.......... 39

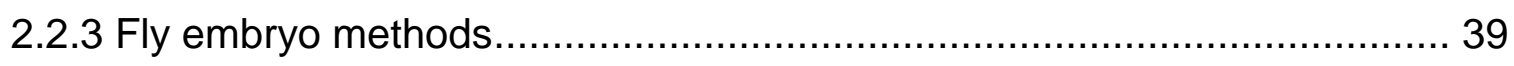

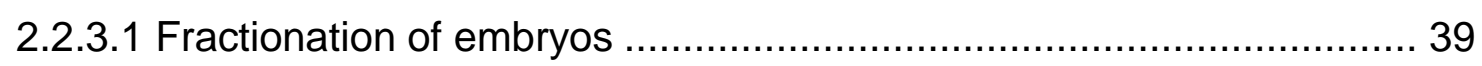

2.2.3.2 RNA isolation following fractionation of embryos ............................... 40

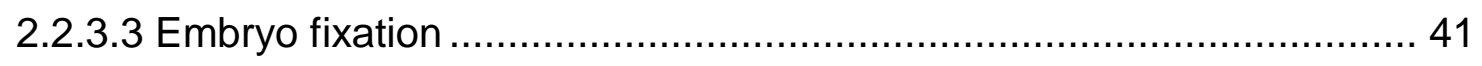

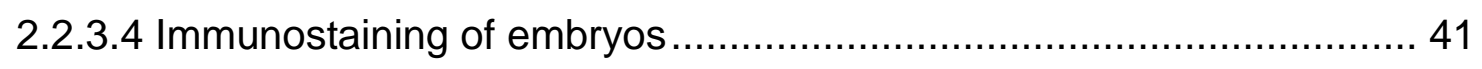

2.2.3.5 Fluorescence in-situ hybridisation (FISH) .................................... 41

2.2.3.6 Generation of germline clones ................................................... 43

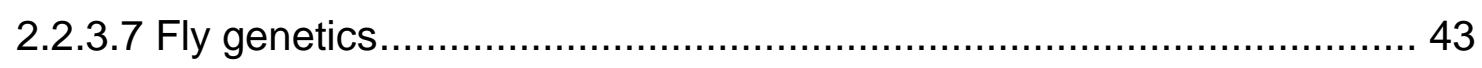

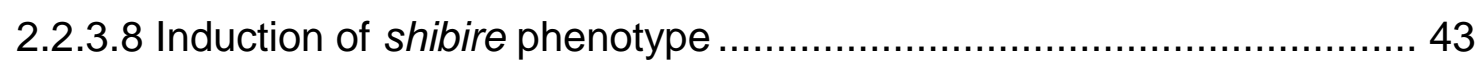

2.2.3.9 Microinjection of protein/drug into embryos .................................... 44

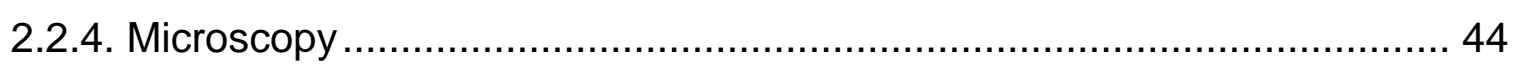

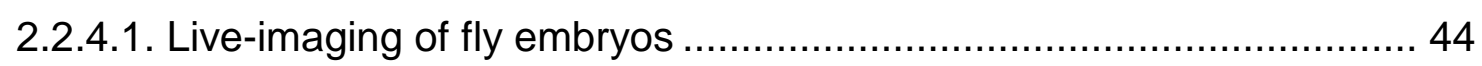

2.2.4.2 Fluorescence recovery after photobleaching (FRAP) ........................ 44

2.2.4.3 Laser ablation of centrosomes ....................................................... 44 
3. RESULTS.

3.1 Factors contributing to the accumulation of Slam protein at the furrow canal . 46

3.1.1 Centrosomes define the site of invagination ........................................... 46

3.1.2 Accumulation of Slam is dependent on vesicular transport...................... 50

3.2 Dynamics of Slam protein, mobility and life-time …..................................... 59

3.2.1 A fraction of Slam is membrane-associated ......................................... 59

3.2.2 Slam protein is stable during cellularisation .......................................... 60

3.2.3 Mobility of Slam is independent of new translation................................. 63

3.2.4 Mobility of Slam is not directly affected by the recycling endosome.......... 64

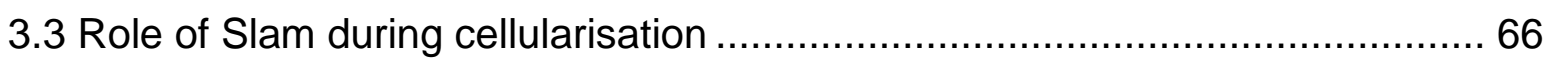

3.1.1 Additional factors apart from Slam are needed for furrow specification ....66 66

3.3.1 nullo and slam together control the specification of the cleavage furrow .. 67

3.3.2 spire - an interactor of slam in yeast two-hybrid screen ......................... 70

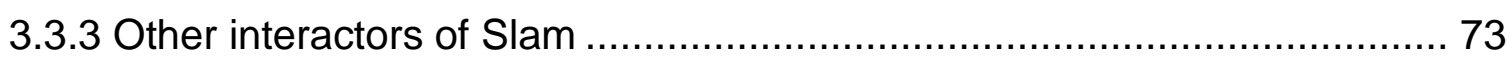

3.4 Slam protein properties and the RNP complex.......................................... 74

3.4.1 Slam has a predicted structured $\mathrm{N}$-terminal half .................................. 74

3.4.2 GFP tag at the N-terminus of Slam partially interferes with its function..... 75

3.4.3 Slam protein and slam mRNA are present in a complex......................... 76

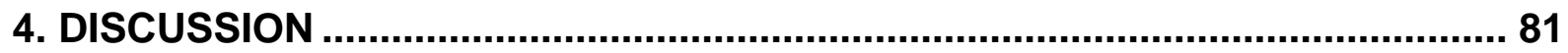

4.1 Centrosomes specify the site of cleavage furrow and restrict Slam to the furrow canal

4.2 Slam restriction at the furrow canal is dependent upon the recycling

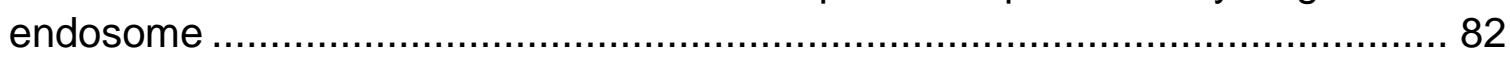

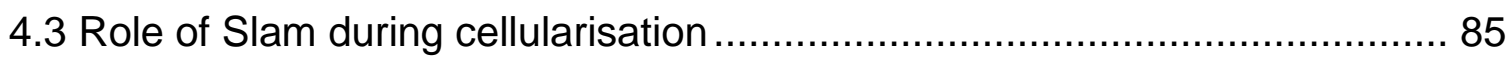

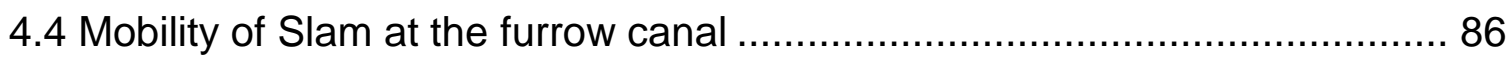

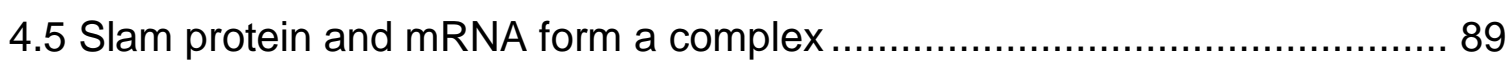

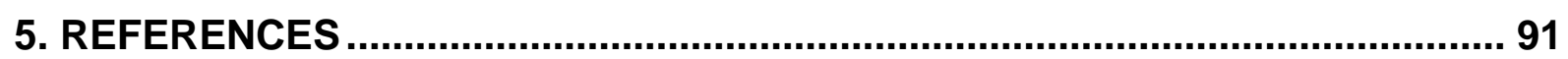




\section{ACKNOWLEDGEMENTS}

I would firstly like to thank my supervisor Prof. Jörg Großhans for allowing me to carry out my $\mathrm{PhD}$ work in his laboratory and for always being available for discussions and to answer questions, no matter how simple (or stupid). I really appreciate his patience, optimism and politeness towards his students. His hardworking nature and passion for science are truly inspiring.

I am very thankful to my thesis committee members Prof. Reinhard Schuh and Prof. Henning Urlaub for their helpful suggestions and constructive criticism during the thesis committee meetings. Their positivity was always very encouraging.

I express my gratitude to my current and past colleagues for creating an excellent working atmosphere, providing with great discussions and German lessons over coffee and fly-sorting. I would especially like to thank everyone involved in the 'Slam project' - Dr. Shuling Yan, Dr. Philip Laupsien, Dr. Christian Wenzl and Stephanie Gröning for sharing results, exciting discussions and helping me with the methods. Without their cooperation, it would have been impossible to carry out this collaborative work. I am grateful to Dr. Maike Claußen for helping me with the EMSA experiments and for suggestions regarding the project. I thank Olaf Bernhard from Dr. Bernhard Schmidt's lab for carrying out Mass spectrometric analysis for us.

I am thankful to all members of the Department of developmental biochemistry for their support and cooperation (and cakes). I could not have asked for a better working environment.

I am indebted to my parents who always believed in me and supported me through thick and thin. The seeds of scientific enquiry and critical thinking sowed by my father and my mother's practical advice and math lessons were crucial to reach this stage. I am thankful to my brother Sreekanth Acharya for being there for me whenever I felt downtrodden for any reason.

I would like to thank Roman Petrovsky for fascinating discussions, insightful comments and constant support in the lab as well as outside of it.

Last but not the least, I would like to thank Mansi Karkhanis for ceaseless support, constant encouragement and general suggestions. 


\section{SUMMARY}

Embryogenesis of many insects starts with a syncytial stage characterised by 13 rapid nuclear divisions. During the following interphase, the plasma membrane of the embryo invaginates between each nucleus to give rise to the first epithelial layer of the embryo. This type of cytokinesis during interphase has been named cellularisation. Two important processes involved in cellularisation are a) specification of the site of cleavage furrow and b) initiation and maintenance of membrane invagination. We investigated the role of a gene slam in these processes. Although it has been shown earlier that slam is necessary for cellularisation and the formation of the basal domain of the invaginating membrane (furrow canal), it has remained unclear how Slam reaches the furrow canal and interacts with the membrane. The aim of the study was to uncover the mechanism of Slam accumulation at the furrow canal, elucidate its mobility dynamics at the membrane and elaborate its role in cellularisation.

By means of live imaging and centrosome ablation, I could show that centrosomes are the initial source of signals for the accumulation of Slam. Using shibire temperature-sensitive mutants, I found that the initial accumulation of Slam at the new furrow was vesicle-dependent but its maintenance at the old furrow was not. Analysis of nuf mutants revealed that although Slam is not directly transported on Rab11-positive vesicles, its proper targeting to the basal domain is indirectly dependent upon the recycling endosome. I identified a role of slam together with another gene nullo in establishing the furrow. I found that slam and nullo act redundantly to each other. Furthermore, using fluorescent recovery after photobleaching experiments, I could show that membrane-associated Slam undergoes a switch-like change from high mobility at the onset of cellularisation to low mobility at mid-cellularisation. Slam mobility in mid-cellularisation is independent of new translation and vesicular trafficking. Finally, I showed that Slam is a ribonucleoprotein complex (RNP) and that slam mRNA was more enriched at the membrane.

I propose that the recycling endosome that is organised by the centrosome restricts a potential Slam receptor to the prospective basal domain of the membrane to which the Slam RNP is recruited from the cytoplasm. Once at the furrow, slam acts together with nullo to establish the furrow and initiate cellularisation without further recruitment of Slam RNP and Slam protein synthesis. 


\section{LIST OF FIGURES}

Fig 1.1 Schematic representation of Drosophila early development $\quad 1$

Fig 1.2 Schematic representation of pseudocleavage furrow formation 3 and cellularisation

Fig 1.3 Schematic representation of Rappaport and cleavage furrows 5

Fig 3.1 Lonesome centrosomes are capable of specifying ectopic furrows 47

Fig 3.2 SAS6GFP overexpression results in extra centrosomes in the 48 embryo due to additional centrosomal divisions

Fig 3.3 GFPslam accumulates around the additional duplicate/singular lonesome centrosomes to form ectopic furrows in SAS6GFP embryos

Fig 3.4 Functional ablation of a centrosome induces loss of its furrow- 50 specifying ability

Fig 3.5 Slam accumulation at the furrow canal is dependent on vesicular 51 transport

Fig 3.6 Loss of Slam from the furrow canal is a specific effect

Fig 3.7 Rab11 is associated with the perinuclear recycling endosome and the recycling endosome vesicles

Fig 3.8 Slam is not transported on Rab11 vesicles

Fig 3.9 Graph depicting the variability of nuf embryos

Fig 3.10 nuf embryos show variable phenotype ranging from mild to severe

Fig 3.11 Slam is not restricted to the basal domain in nuf embryos.

Fig 3.12 Slam mislocalisation corresponds to the severity of nuf phenotype

Fig 3.13 Interference with Rab11 function in the embryo causes delay in Slam restriction to the furrow canal

Fig 3.14 Slam is a membrane-associated protein

Fig 3.15 Effect of blocking protein translation in different stages of embryo

Fig 3.16 Slam protein is stable during cellularisation

Fig 3.17 Mobility of Slam during cellularisation is independent of new translation 
Fig 3.18 Slam is stably associated to the membrane during interphase 13 and 14 and switches to a high-mobility state at the onset of cellularisation

Fig 3.19 Slam mobility is slightly increased in a subset of nuf embryos

Fig 3.20 The mobility of Slam during cellularisation is not dependent on vesicular trafficking

Fig 3.21 Schematic representation of alleles of slam

Fig 3.22 Furrow specification requires factors additional to slam

Fig 3.23 nullo and slam together control the specification of the furrow

Fig 3.24 Schematic representation of interaction of Slam and Spire in a 70 Yeast two-hybrid screen

Fig 3.25 spir alleles show cell-cycle defects and occasional cellularisation defects

Fig 3.26 Spire antibody against the KIND domain detects several isoforms of Spire

Fig 3.27 Immunostaining against Spire shows apical staining during 73 cellularisation

Fig 3.28 Pulldown of Slam and GFPslam

Fig 3.29 Schematic representation of disordered regions in Slam protein

Fig 3.30 GFP tag partially interferes with Slam function

Fig 3.31 GFPslam is less efficiently bound to the membrane 76

Fig 3.32 slam mRNA and protein are present in a complex 77

Fig 3.33 slam mRNA is enriched at the membrane 78

Fig 3.34 slam mRNA and protein also colocalise ectopically 79

Fig 3.35 Binding assay with Slam $\Delta$ C651 protein and slam mRNA localising elements

Fig 4.1 Model for the role of centrosomes in targeting of recycling endosome vesicles to the site of cleavage furrow

Fig 4.2 Mobility of Slam and the role of recycling endosome in its restriction 


\section{LIST OF TABLES}

Table 2.1 Oligonucleotides used in the study 25

Table 2.2 Plasmids used in the study 25

Table 2.3 Antibodies used in the study 26

$\begin{array}{lll}\text { Table 2.4 Fly stocks used in the study } & 27\end{array}$

$\begin{array}{lll}\text { Table 3.1 Penetrance of cell-cycle and cellularisation defects in } & 72\end{array}$ different spire alleles

Table 3.2 qRT-PCR threshold cycle (Ct) numbers for three 79 independent fractionation experiments 


\section{LIST OF MOVIES}

Movie 1 Extra centrosome replication (Fig 3.2)

Movie 2 Duplicate lonesome centrosomes (Fig 3.3 A)

Movie 3 Singular lonesome centrosome (Fig 3.3 B)

Movie 4 Centrosome ablation (Fig 3.4)

Movie 5 GFPslam control $32^{\circ} \mathrm{C}$ (Fig 3.5)

Movie 6 GFPslam in shibire $32^{\circ} \mathrm{C}$ (Fig 3.5)

Movie 7 GFPslamRab11YFP (Fig 3.8)

Movie 8 GFPslam WT (Fig 3.11)

Movie 9 GFPslam nuf embryo 1 (Fig 3.11)

Movie 10 GFPslam nuf embryo 2 (Fig 3.11)

Movie 11 GST injected in GFPslam embryo (Fig 3.13 A)

Movie 12 Rab11S25N injected in GFPslam embryo (Fig 3.13A)

Movie 13 Buffer injected into hisRFP embryo (Fig 3.15A)

Movie 14 Cycloheximide into hisRFP embryo (Fig 3.15A)

Movie 15 Buffer injected into GFPslam embryo at anaphase of cycle 13 (Fig 3.15B)

Movie 16 Cycloheximide injected into GFPslam embryo at anaphase of cycle 13 (Fig 3.15B)

Movie 17 Buffer injected into GFPslam embryo at onset of cycle 14 (Fig 3.15C)

Movie 18 Cycloheximide injected into GFPslam embryo at onset of cycle 14 (Fig 3.15C)

Movie 19 Buffer injected into GFPslam embryo at mid-cellularisation (Fig 3.15D)

Movie 20 Cycloheximide injected into GFPslam embryo at midcellularisation (Fig 3.15D)

Movie 21 GFPslam FRAP in buffer injected embryos (Fig 3.17) 
Movie 22 GFPslam FRAP in cycloheximide injected embryos (Fig 3.17)

Movie 23 GFPslam FRAP in WT background (Fig 3.19 A)

Movie 24 GFPslam FRAP in nuf embryo1 (Fig 3.19C)

Movie 25 GFPslam FRAP in nuf embryo2 (Fig 3.19E)

Movie 26 GFPslam FRAP in shibire (Fig 3.20) 


\section{ABBREVIATIONS}

aa

B-gal

bp

cDNA

DAPI

$\mathrm{dd}_{2} \mathrm{O}$

${ }^{\circ} \mathrm{C}$

DNA

DTT

$\Delta$

$\Delta \mathrm{C}$

E.coli

EDTA

EMSA

FRT

FRAP

GEF

GFP

GST

g

glc

$\mathrm{h}$

IPTG

$\mathrm{kb}$

$\mathrm{kDa}$

I

m

$\mu$

$\min$

mRNA amino acid(s)

beta-galactosidase

base pairs

complementary DNA

4', 6' - Diamidino-2-phenylindole

double distilled water

degree Celsius

deoxyribonucleic acid

1,4-dithiothreitol

deletion

C-terminal deletion

Escherichia coli

ethylenediaminetetraacetic acid

electrophoretic mobility shift assay

flippase recognition target

fluorescence recovery after photobleaching

guanidyl nucleotide exchange factor

green fluorescent protein

Glutathion-S-transferase

gram(s)

germline clone

hour(s)

Isopropyl-B-D-thiogalactopyranoside

kilobases

kiloDalton

litre(s)

milli-

micro-

minute(s)

messenger RNA 
$\mathrm{Nr}$

PCR

PMSF

RNA

RNAi

RNP

rpm

RT

SDS

SDS-PAGE

Tris

WT

w

$\mathrm{Y} 2 \mathrm{H}$ number

polymerase chain reaction

Phenylmethylsulfonylfluorid

ribonucleic acid

RNA interference

ribonucleoprotein

revolutions per minute

room temperature

sodiumdodecylsulphate

SDS-polyacylamide gel electrophoresis

tris(hydroxymethyl)aminomethne hydrochloride

wild-type

white gene

yeast two-hybrid 


\section{INTRODUCTION}

\subsection{Drosophila early development}

The early embryonic divisions of a Drosophila embryo, similar to many insects, take place in a syncytium. Subsequent to fertilisation of the oocyte by a sperm, the nucleus undergoes thirteen rounds of mitotic divisions (Zalokar and Erk, 1976; Foe and Alberts, 1983). However, the nuclear divisions are not accompanied by cytokinesis of the embryo, resulting in the daughter nuclei lying in a common cytoplasm. The first eight mitotic cycles take place in the embryonic core and are referred to as the preblastoderm cycles. These cycles are very rapid, lasting 8 min on an average and consist only of the synthetic phase (S-phase) and the mitotic phase (M-phase) of cell cycle (Zalokar and Erk, 1976). From cycle 9 onwards, most of the nuclei undergo microtubule-dependent cortical migration, which involves their migration from the centre of the embryo to the cortex. This stage of the embryo where the embryo contains a cortical monolayer of nuclei in a syncytium is called syncytial blastoderm. Some

A

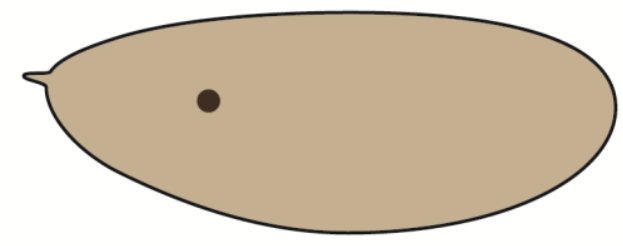

C

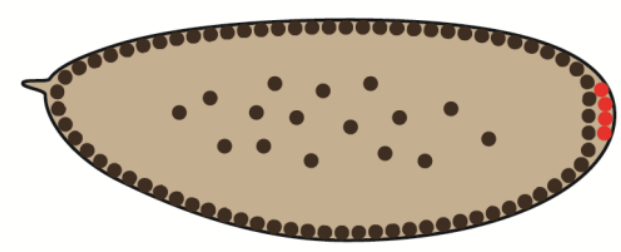

B

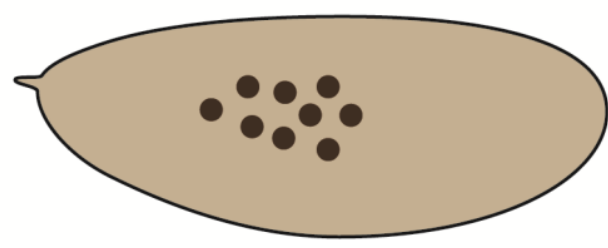

D

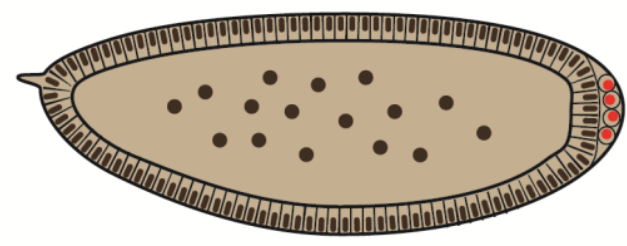

Fig 1.1 Schematic representation of Drosophila early development (A) Embryo after fertilisation (B) The first nine mitotic cycles take place in the core of the embryo (C) From cycle nine, the embryos undergo cortical migration to give rise to a syncytial blastoderm. Some nuclei migrate to the posterior pole to form the pole cells (D) In the interphase of cycle 14, the plasma membrane of the oocyte invaginates and encloses the nuclei into individual cells to form the cellular blastoderm. 
nuclei migrate to the posterior pole of the embryo to form the pole cells, which are germ cell precursors. The nuclei at the cortex remain anchored beneath the plasma membrane where they undergo further mitotic divisions. These divisions include a second gap phase (G2 phase), whose length progressively increases following each cycle. After the end of the thirteenth cell cycle, there is an extended interphase of cycle 14, which lasts about an hour (Foe and Alberts, 1983). During this interphase, the plasma membrane of the embryo starts invaginating between each nucleus and progressively encloses each nucleus into an individual cell, in a process called cellularisation (Fig 1.1).

The cortex of the embryo is rich in actin that undergoes a cycle of redistribution under the influence of centrosomal asters during the syncytial cycles 10 to 13 (Kao and Megraw, 2009). During interphases, it accumulates as actin caps above each nucleus. As the nuclei progress through prophase and enter metaphase, the plasma membrane of the embryo invaginates between adjacent nuclei, reaching a depth of up to approximately $8 \mu \mathrm{m}$. These structures are called pseudocleavage furrows or metaphase furrows. Metaphase furrows prevent fusion of spindle from adjacent nuclei (Sullivan et al., 1993). As the cell cycle approaches telophase, the metaphase furrows start regressing and the actin rearranges itself again as actin caps. This cycling of actin distribution occurs until the embryo reaches the stage of cellularisation during which actin persists at the tip of the invaginating membrane (Fig 1.2).

At the onset of cellularisation during the cycle 14 interphase, microtubules emanate from the apically-located centrosomes. These microtubules extend downwards to encompass the nuclei into an inverted basket-like structure and are necessary for furrow invagination.

The process of cellularisation has been divided into four distinct phases on the basis of morphology and speed of invagination of the membrane (Knoblich, 2000; Lecuit and Wieschaus, 2000). At Phase one, the cortical region above each nucleus is rich in microvilli while the region at the middle of adjacent nuclei, which is called furrow canal, is devoid of microvilli (Lecuit and Wieschaus, 2000). Furrow canals form the leading edge of the invaginating furrows (Fullilove and Jacobson, 1971). Phase one, which lasts about 10 minutes, is also accompanied by nuclear elongation. Phase two is characterised by the completion of nuclear elongation but very slow membrane 
A

B
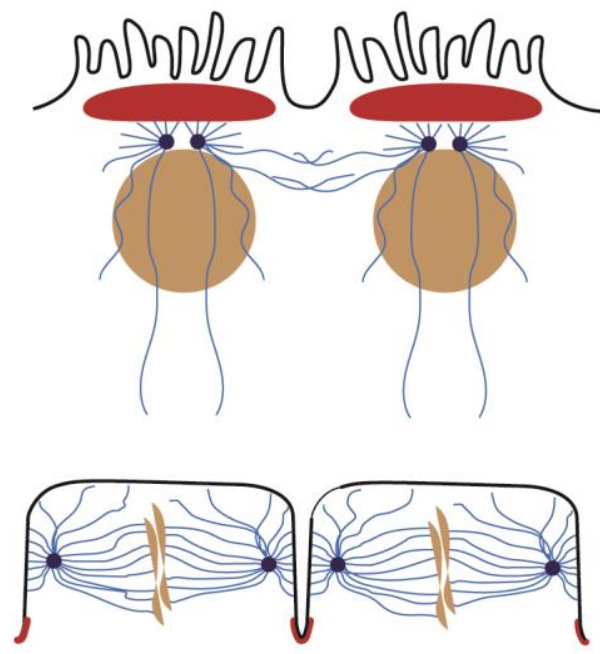

C

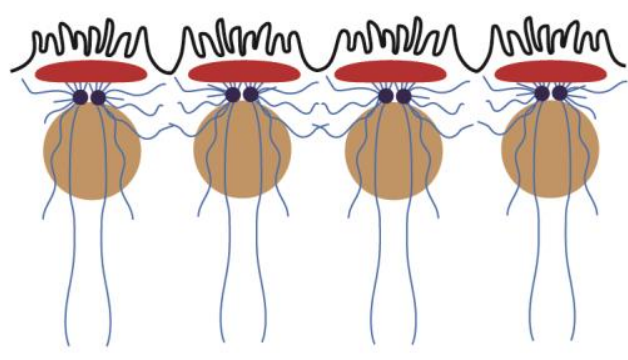

D

E
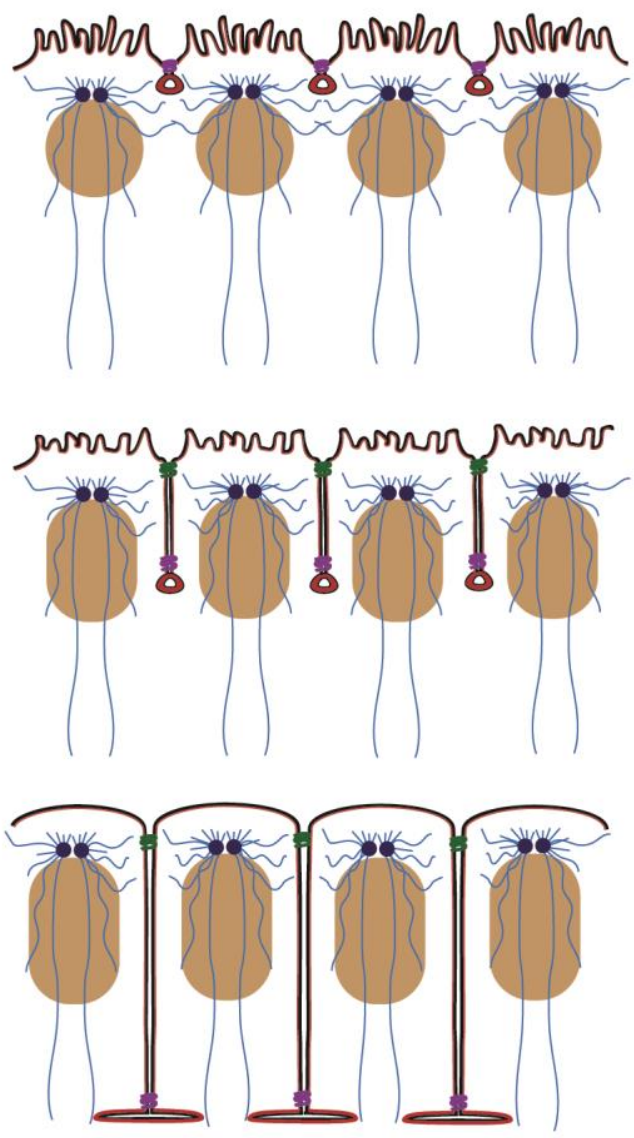

- Centrosome $\approx$ Microtubules

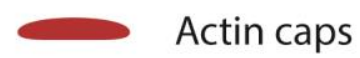

- $\quad$-actin at FC
Apical adherens junctions

Basal adherens junctions

Fig 1.2 Schematic representation of pseudocleavage furrow formation and cellularisation. (A) Cycle 13 interphase: actin is located apical to the nuclei as caps (B) Cycle 13 mitosis: Metaphase furrows are formed and actin redistributes itself to the tip of the furrows (C) Cycle 14 interphase: actin forms caps again (D) Onset of cellularisation: Furrow canal and basal adherens junctions are formed, actin redistributes itself to the furrow canal $(E, F)$ apical adherens junctions are formed and cellularisation proceeds first slowly (slow phase) and then the rate speeds up (fast phase).

invagination. In phase three, the invaginating membrane reaches to the plane of the basal region of the nuclei. When the membrane reaches about $35 \mu \mathrm{m}$ in depth, it starts contracting laterally in a process called basal closure, which encloses each nucleus from beneath to give rise to blastoderm cells. Cellularisation is a precisely regulated process requiring establishment and maintenance of membrane polarity, specification of the cleavage site, regulated membrane growth and finally basal closure to enclose 
the nuclei into cells. These processes are discussed in further detail in the following sections.

Cellularisation involves formation of a polarised epithelium. As the plasma membrane invaginates during this process, it becomes compartmentalised into apical, lateral and basal domain. It has been shown that the plasma membrane of the embryo displays a polarised organisation already during the syncytial blastoderm stage (Mavrakis et al., 2009). Two distinct plasma membrane domains have been identified during the syncytial cycles a) above each nucleus and b) at the lateral region of the nuclei. These regions contain distinct membrane markers and are unable to diffuse outside of their own domain, indicating towards the existence of a diffusion barrier. This diffusion barrier was shown to be actin-dependent (Mavrakis et al., 2009).

Establishment and maintenance of membrane polarity during cellularisation is also dependent upon several other factors such as proper vesicle targeting through the recycling endosome and the proper localisation of initial furrow canal markers such as Actin and Slam. The role of these factors has been discussed later under individual sections.

\subsection{Cleavage furrow site specification}

Cellularisation of the Drosophila embryo is a modified cytokinesis and hence, many of the mechanisms involved in the positioning of the cytokinetic furrow during cellularisation are similar to conventional cytokinesis. The cytokinetic furrow in eukaryotic animal cells is positioned at the cell cortex midway down the longitudinal axis of the mitotic spindle (Balasubramanian et al., 1992; Bi et al., 1998; Fujiwara and Pollard, 1976; Mabuchi and Okuno, 1977, 1977). The positioning of the furrow is carried out by the central spindle or astral microtubules, depending on the cell size and type. Rappaport's classic experiments with marine invertebrate embryos suggested that astral microtubules are the source of the furrow initiation signal. He created a binucleate embryo artificially and found that when these nuclei divided, apart from the two furrows that formed between the respective metaphase plates, a third ectopic furrow formed between neighbouring centrosomes in the absence of a nucleus or a central spindle (Rappaport, 1961). He proposed that the signal for initiation of the ectopic furrow must be generated by overlapping astral microtubules (Fig 1.3). It was further demonstrated that a minimal distance between the two centrosomes as well as 
between the centrosomes and cortex were essential for furrow induction (Rappaport, 1986).

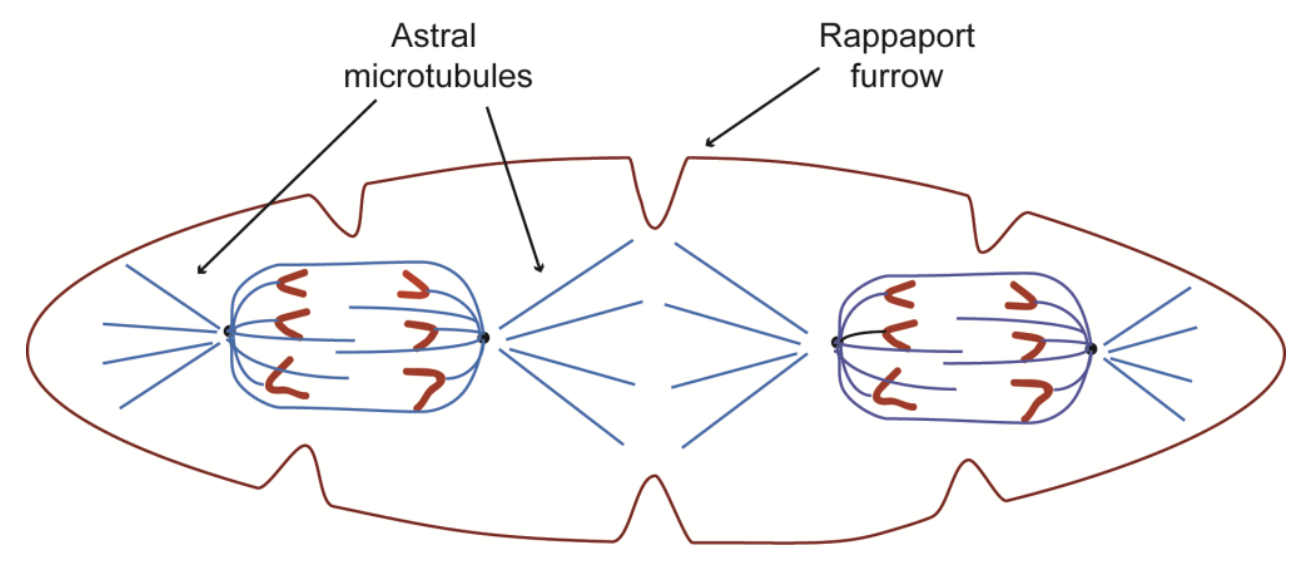

Fig. 1.3 Schematic representation of Rappaport and cleavage furrows. Artificially induced Rappaport furrows are formed between overlapping asters of adjacent centrosomes while cleavage furrows are formed between the overlapping spindles between a centrosome pair (Adapted from Sullivan, 2009).

However, studies in cultured cells have suggested that the region of overlapping antiparallel microtubules called the central spindle is responsible for furrow positioning (Wheatley and Wang, 1996; Williams et al., 1995). A number of proteins required for cytokinesis are recruited to the central spindle. Rappaport had also showed that flattening the sand dollar embryos so as to allow the central spindle to interact with the cortex could induce a furrow (Rappaport, 1985). Recent studies on Caenorhabditis elegans (C.elegans) embryos have suggested both astral microtubules as well as the central spindle contribute to furrow induction (Bringmann and Hyman, 2005; Motegi et al., 2006). In these embryos, it seems that the initial furrow induction signal comes from the asters, while the completion of cytokinesis depends on the sustenance of the signal from the central spindle (Baruni et al., 2008; Bringmann and Hyman, 2005).

In addition to forming the astral microtubules, other centrosome-associated activities are also essential for furrow specification. The Drosophila Centrosomin $(\mathrm{CNN})$ is a core centrosomal protein required for normal pericentriolar material organisation and astral microtubule assembly (Li and Kaufman, 1996; Megraw et al., 1999). A hypomorphic allele of $c n n$ called $c n n^{B 4}$ has been used to dissect these two 
functions since this allele shows severe disruptions in furrow formation, despite having an apparently normal microtubule organisation (Kao and Megraw, 2009). Kao and Megraw identified another protein Centrocortin (CEN), which interacts with CNN. CEN partially colocalises with CNN at the centrosomes and is also found on cleavage furrows. Strong cen alleles show similar phenotype as $c n n^{B 4}$ allele where cleavage furrows are weak or broken. Like $c n n^{B 4}$, cen mutants show no discernable defects in microtubule organisation. This study demonstrates the role of centrosomal protein CNN in relaying a furrow initiation signal to via CEN, thus suggesting that centrosomes possess additional information for furrow specification which is independent of astral microtubule assembly.

Various studies have been done suggesting centrosomes are capable of determining the site of furrow invagination independent of the nuclei. Anucleate C.elegans embryos containing only a pair of centrosomes are able to form a cytokinetic furrow and attempt to divide (Baruni et al., 2008). In Drosophila syncytial embryos, it has been shown that if centrosome and nuclear divisions are uncoupled by inhibiting S-phase, and nuclear migration to the cortex prevented, the centrosomes that migrate to the posterior pole are able to initiate pole cell formation in the absence of nuclei (Raff and Glover, 1989). In another study, blocking nuclei from entering mitosis by simultaneous RNAi of all three mitotic cyclins in the early Drosophila embryos showed that centrosomes are able to organise Myosin 'cages' around them (McCleland and O'Farrell, 2008). The timing of cellularisation was unaffected despite the nuclear arrest (McCleland and O'Farrell, 2008) which suggests that the temporal information for the initiation of cellularisation is not regulated by the number of nuclear divisions.

In summary, positioning of the furrow is controlled by astral microtubules or central spindle or both depending upon the size and type of the cell. Centrosomal activity, in addition to its microtubular organizing capacity, is required during cellularisation in Drosophila embryos. Also, centrosomes have the ability to organise the furrow position in the absence of nuclei in several systems.

\subsection{Initiation and maintenance of membrane invagination}

Several maternal as well as zygotic genes are necessary for the initiation of membrane invagination. Some of them are discussed below. 


\subsection{1 slow as molasses (slam)}

One of the zygotic genes that plays an important role in the initiation of membrane invagination is slow as molasses (slam). slam is highly expressed the onset of cellularisation. Zygotic deficiency of slam shows a very strong and penetrant cellularisation phenotype (Lecuit et al., 2002). It showed defects primarily in the slow phase of cellularisation causing delayed furrow invagination, which is why it was named 'slow as molasses' originally (Lecuit et al., 2002). However, removal of both maternal and zygotic contributions has demonstrated that slam is in fact essential for furrow invagination. Embryos devoid of maternal and zygotic slam display no furrow invagination (Stein et al., 2002; Acharya et al., 2014; Dr. Philip Laupsien, PhD dissertation).

Already at the onset of cellularisation, the respective morphologies of the apical and the basal regions of the plasma membrane are distinguishable. The apical part, which lies above the nuclei, consists of many villous projections. Adjacent to this region is a region of depressed plasma membrane that lacks villous projections. As the slow phase progresses, formation of the basal junction brings together the adjacent lateral regions of the plasma membrane, thus delimiting the basal domain from the lateral domain. In slam zygotic deficiency embryos, although the prospective site of furrow invagination shows a slight depression, no basal junction is formed and the villous projections extend into the prospective furrow canal (Lecuit et al., 2002). Despite the abnormal furrow canal organisation, a delayed membrane invagination can be seen in the presence of maternal Slam contribution (Lecuit et al., 2002). Membrane invagination is completely abolished only when the maternal contribution is also removed (Acharya et al., 2014; Dr. Philip Laupsien, PhD dissertation). This suggests that Slam has a dual role - one in polarity establishment and second in membrane invagination during cellularisation.

The apical domain of the plasma membrane rich in villous projections shows higher endocytic behaviour compared to the prospective furrow canal region (Lecuit and Wieschaus, 2000). slam zygotic deficiency embryos show reduced endocytosis at the apical region (Lecuit et al., 2002). This suggests that Slam might establish the polarity of the membrane by regulating transcytosis at the onset of cellularisation (Lecuit et al., 2002). However, Slam localises exclusively at the furrow canal (Grosshans et al., 2005; Lecuit et al., 2002) so it is unclear how exactly Slam might regulate endocytosis at the apical region. 
Zygotic expression of hypomorphic alleles of slam (slamwaldo1 and slamwaldo2) does not show a cellularisation phenotype but instead display germ cell migration defects. During the process of germ cell migration, neither slam mRNA nor the protein are detectable in the embryo. Therefore, the mechanism behind the role of slam in germ cell migration is unknown. It has been speculated that during cellularisation itself, Slam might be recruiting factors necessary for the subsequent germ cell migration.

\subsection{2 nullo}

nullo was first identified as a locus on the $\mathrm{X}$-chromosome that was necessary for cellularisation (Rose and Wieschaus, 1992; Simpson and Wieschaus, 1990; Wieschaus and Sweeton, 1988). In nullo mutant embryos, the cleavage furrow is unable to invaginate resulting in the generation of multinucleate cells. Nullo stabilises the accumulation of components of the basal junction; in nullo mutants, Armadillo and E-cadherin are not concentrated in the junctional region and are instead spread across the lateral membrane (Hunter and Wieschaus, 2000). It was suggested that due to the failure of formation of the basal junction, compartmentalisation of the invaginating membrane was compromised. However, subsequent studies found that furrow compartmentalisation does not require the basal adherens junctions and that rather the reduced F-actin levels at furrow canals in nullo mutants is the cause of polarity disruption (Sokac and Wieschaus, 2008b).

Nullo protein is rapidly degraded before the completion of cellularisation and apical adherens junction formation. Prolonging the expression of Nullo into late cellularisation causes blockage of the apical clustering of Armadillo, $\alpha$-catenin and Ecadherin. These junctional defects cause a disrupted cell morphology resulting in a failure to form the ventral furrow. These studies indicate that establishment of the apical junction and the formation of the ventral furrow is dependent on the rapid degradation of Nullo in late cellularisation (Hunter and Wieschaus, 2000).

Nullo contains an $\mathrm{N}$-terminal myristoylation site that is important for its targeting to the plasma membrane during cycle 14. Interestingly, Nullo protein devoid of the myristoylation site still shows normal localisation to the plasma membrane of the metaphase furrows during cycle 13 (Hunter et al., 2002). This suggests that at the onset of cellularisation, there is alteration of some aspect of Nullo targeting or membrane association. 
Another gene that shows similar multinucleate phenotype as nullo mutants is serendipity- $\alpha$ (sry- $\alpha$ ) (Merrill et al., 1988; Schweisguth et al., 1990). nullo mutants show severely impaired localisation of Sry- $\alpha$ at the furrow canal and the basolateral membrane (Postner and Wieschaus, 1994; Schweisguth et al., 1990). Therefore it is likely that nullo and sry- $\alpha$ act in the same pathway where nullo acts upstream to $s r y-\alpha$.

\subsubsection{RhOGEF2}

RhoGEF2 is a guanine triphosphate (GTP) exchange factor which is localised apically in epithelial cells throughout embryogenesis. During cellularisation, RhoGEF2 is localised at the furrow canal. RhoGEF2 germline clones show defects in cellularisation with furrow canals often missing between adjacent nuclei, resulting in multinucleate cells. During later stage of cellularisation, irregularities in nuclear arrangement were observed. Analysis of the furrow canal morphology through electron microscopy revealed that the furrow canals are often enlarged and do not retain the typical hairpin loop structure. RhoGEF2 germline clones however, show normal rate of membrane invagination (Grosshans et al., 2005). This is in contrast to slam where removal of either the maternal or the zygotic contribution causes reduction in the rate of membrane invagination and no membrane invagination occurs in the slam null situation (Acharya et al., 2014, Dr. Philip Laupsien, PhD dissertation). RhoGEF2 recruitment to the furrow canal is however, dependent upon Slam. Slam is necessary and sufficient for the localisation of RhoGEF2 (Wenzl et al., 2010).

RhoGEF2 genetically interacts with Rho1 and acts as its specific positive regulator during cellularisation (Barrett et al., 1997; Grosshans et al., 2005). Rho1 is a small GTPase that plays an important role in the regulation of the actomyosin cytoskeleton (Allen et al., 1997). Rho1 also localises to the furrow canal where Diaphanous and Myosin II are its two key targets. Diaphanous is an actin-nucleator while non-muscle Myosin II is an actin-binding protein that can cross-link actin and regulate contractility. Therefore, RhoGEF2 regulates the actomyosin network at the furrow canal via Rho signalling through downstream effectors such as Diaphanous and Myosin II. 


\subsection{4 diaphanous (dia)}

Dia belongs to a family of proteins called Formins, which associate themselves at the plus end (barbed end) of an actin filament and mediate nucleation and polymerisation (for a review, see Chesarone et al., 2010). In addition, it has been proposed that Dia also regulates microtubule stability (Ishizaki et al., 2001; Palazzo et al., 2001; Wen et al., 2004). Consistent with its function, dia hypomorphic embryos display severe morphological defects after nuclear cycle 11. Actin is absent from the hexagonal arrays of metaphase furrows. Two other furrow components, Anilin and Peanut (a Drosophila septin), also fail to recruit to the metaphase furrows. Additionally, Myosin II accumulation at the metaphase furrows is also weak (Afshar et al., 2000).

During cellularisation, Dia localises to the furrow canal. dia embryos show variable defects in actin and microtubule organisation during this stage. The least severe cases show morphological and positional defects in nuclei or microtubular structure. A more severe phenotype is characterised by irregularities or absence of Actin localisation at the furrow canals. Also, the positioning of nuclei and their associated microtubular baskets is affected (Afshar et al., 2000).

Dia also promotes stability of the basal domain by suppressing endocytosis at the region. It has been proposed that the linear actin filaments generated by Dia form a dense cortical layer giving rise to mechanical constraints which prevent endocytic budding. dia embryos show spreading of the lateral membrane markers such as Discslarge (Dlg) and Armadillo/ß-Catenin (Arm) into the basal domain suggesting that Dia plays a role in the establishment of baso-lateral polarity (Yan et al., 2013).

\subsection{5 abelson and enabled}

abelson (abl) is a gene encoding a highly conserved nonreceptor tyrosine kinase which regulates cortical actin (for a review, see Van Etten, 1999). ab/M mutants (embryos lacking maternal contribution while half of the embryos also lack zygotic contribution) display defects or absence of metaphase furrows in cycle 13. During cellularisation, they show multinucleate cells with abnormal microtubule baskets. In these embryos, actin is reduced at the metaphase furrows while it shows excess accumulation at the apical region. Abl is localised in the syncytial blastoderm to the Actin caps and metaphase furrows. It has been shown that Abl regulates actin polymerisation primarily by downregulating another protein Enabled (Ena) through an 
unknown mechanism (Grevengoed et al., 2003). Ena belongs to a family of actin modulators Ena/VASP Homology proteins (Ena/Vasodilator-stimulated phosphoproteins) which are involved in cell motility (for a review, see (Krause et al., 2002). Ena/VASP proteins promote the growth of filamentous actin, possibly by binding to the barbed end and negatively regulating capping protein that restrains polymerisation (Bear et al., 2002). ab/M mutants show excess accumulation of Ena at the apical region which is the likely cause of excessive Actin accumulation. ena deficient embryos however, show no defects in the hexagonal actin arrays during cellularisation (Fox and Peifer, 2007). Also, Ena is not localised at the furrow canal which suggests that it probably does not have a direct role in actin organisation at the furrow canal. Other pathways for actin polymerisation at the furrow canal, one of them being the Rho1-Diaphanous pathway are more directly involved. Arp2/3-mediated (Actin-related proteins 2/3) branched actin polymerisation has also been implicated to have some, but not a major role in the organisation of f-actin at the furrow canal (Stevenson et al., 2002).

\subsubsection{Cytoskeletal components}

A proper cytoskeletal organisation is essential for cellularisation. All the genes mentioned above that disrupt cellularisation act via directly or indirectly affecting the cytoskeleton. Therefore it is expected that mutating/downregulating cytoskeletal components would disrupt cellularisation. The role of cytoskeleton during syncytial divisions and cellularisation has been long established (Foe and Alberts, 1983; Foe et al., 1993). Intact microtubules, microfilaments and f-actin are necessary for cellularisation. When microtubules are depolymerised by injection of Colcemid in embryos at the onset of cellularisation, the plasma membrane invagination is abolished. However, when injected at the beginning of the fast phase, there is no influence on the invagination. Depolymerisation of microfilaments through injection of Cytochalasin B at the onset of cellularisation causes blockage of membrane invagination and even a likely retraction (Foe et al., 1993). Actin is dynamically associated at the furrow canal as revealed by its quick recovery following photobleaching. Preventing actin polymerisation by injection of Latrunculin A into cellularising embryos caused loss of furrows showing that maintenance of actin at the furrow through continuous polymerisation is necessary to sustain plasma membrane integrity (Cao et al., 2008). 
It is also known that microtubular motors Kinesin and Dynein activity is necessary for furrow formation and membrane invagination. It has been reported that injection of the function-inhibiting anti-Khc (Kinesin1 heavy chain) antibody causes disruption in cellularisation (Susan L. Tran and Michael A. Welte, unpublished - as mentioned in Shubeita et al., 2008). The role of a kinesin-6, Pavarotti-KLP (PavarottiKinesin-like protein; Pav-KLP) has been studied in early Drosophila embryos. In syncytial embryos, Pav-KLP is seen to be localised on the spindle where it colocalises with microtubules. It has been proposed to be involved in the organisation of spindle. Injection of an antibody against Pav-KLP at this stage shows defects in chromosome segregation, spindle collapse and perturbed or absent telophase midbodies. Pav-KLP is also seen to localise at the cortex, colocalising with actin caps. Blocking Pav-KLP function during cellularisation showed reduced furrow invagination. Recruitment of actin at the furrow canal was also lost, thus suggesting that Pav-KLP is involved in actin remodelling (Sommi et al., 2010).

Use of two lethal hypomorphic alleles of Dynein-heavy chain $D h c 64 C^{6-6}$ and Dhc64C6-8 that complement each other has allowed to study the role of Dynein in the early Drosophila embryo. Embryos from Dhc64C6-6/ Dhc64C6-8 adult females lack the maternal contribution of dynein heavy chain. Mitotic defects were observed during the syncitial cycles and majority of the embryos fail to properly cellularise and complete gastrulation (Robinson et al., 1999).

Another gene that has been implicated in furrow canal establishment by regulating Dynein-mediated transport is the MAST kinase Drop out (Dop). In dop mutants, membrane invagination is severely delayed (Hain et al., 2014; Meyer et al., 2006). Membrane polarity is also disrupted as Slam restriction to the furrow canal is delayed and DIg is spread into the basal domain. dop controls phosphorylation of Short wing [Drosophila Dynein intermediate chain (Dic)] either directly or indirectly (Hain et al., 2014). This study shows that dynein-based transport is crucial for cellularisation.

\subsection{Membrane transport}

Addition of membrane to the invaginating furrows is necessary in order for cellularisation to proceed. The apical domain of the plasma membrane is rich in microvilli which unfold as the furrow ingresses (Figard et al., 2013; Figard and Sokac, 2014). Another source of membrane is through transcytosis in which endocytic vesicles 
bud off from the apical domain and fuse to the lateral domain (Lee and Harris, 2013; Pelissier et al., 2003). However, new membrane supply is also provided to the apical region at phase 1 and to the apico-lateral region at phase 2 of cellularisation. Injection of colchicine blocks the transport of Neurotactin, a transmembrane protein that localises apically and laterally. This shows that the transport of secretory membranes from Golgi-apparatus to the ingressing plasma membrane is mediated by microtubules (Lecuit and Wieschaus, 2000). Lava lamp (Lva) is a Golgi-associated coiled coil protein which when inactivated using antibodies, inhibits furrow invagination. Consistent with this observation, injection of Brefeldin A, an inhibitor of Golgi-derived membrane vesicle transport caused inhibition of membrane invagination (Sisson et al., 2000). It has been shown that Lva is necessary for dynein-mediated targeting of the secretory machinery and that it specifically associates with Golgi spectrin as well as dynein, dynactin and cytoplasmic linker protein-190 (CLIP-190) (Papoulas et al., 2005).

Several studies have shown that vesicular trafficking is required for the transfer of membranes and furrow components to the ingressing membrane during cellularisation. Dynamin is a conserved protein that is necessary for the scission of clathrin-coated vesicles (Hinshaw and Schmid, 1995). The Drosophila homologue of dynamin, shibire, is indispensable for cellularisation (Swanson and Poodry, 1981). Using a temperature-sensitive allele, it was shown that shibire is required for membrane invagination in the slow phase of cellularisation (Pelissier et al., 2003). This is due to inhibition of apical endocytosis from the plasma membrane as well as impairment of vesicle trafficking from the trans-golgi network and recycling endosome. Injection of a dominant negative variant of Rab5, an early endosomal protein, leads to reduction of speed of furrow invagination, indicating that vesicle trafficking through the early endosome is also necessary for cellularisation (Pelissier et al., 2003).

The importance of vesicular trafficking for cellularisation is also demonstrated by the necessity of a functional exocyst complex for this process. Exocyst is an octameric protein complex that defines the sites at which vesicles tether and fuse to the plasma membrane during cytokinesis (Finger et al., 1998). It has been shown that Sec5, a component of the exocyst, is necessary for the invagination of cleavage furrows. $\sec ^{\text {ts } 1}$ (a temperature-sensitive mutant allele) embryos display no cleavage furrow invagination and fail to deposit Neurotactin, a transmembrane protein, at the plasma membrane. It has been suggested that exocyst is likely to direct polarised 
addition of new membrane at the apico-lateral region of the invaginating membrane (Murthy et al., 2010).

The recycling endosome is an endosomal compartment located at the pericentrosomal region apical to the nuclei in the Drosophila embryo. Rab11 is a key mediator of the recycling endosome function and is required for the proper targeting of recycling endosome vesicles (Wilson et al., 2005). Interfering with Rab11 function by injecting a dominant negative variant of the protein into cellularising embryos causes inhibition of membrane invagination during slow phase (Pelissier et al., 2003). Rab11 physically interacts with another protein called Nuclear fallout (Nuf), a Rab11 effector needed to maintain the structural integrity of the recycling endosome (Rothwell et al., 1999; Riggs et al., 2003; Horgan et al., 2007). Both Rab11 and Nuf are necessary for each other's localisation at the pericentrosomal region (Riggs et al., 2003). Nuf is a structural and functional homologue of a mammalian ADP ribosylation factor (Arf) effector called Arfo2 (Arfophilin-2) (Hickson et al., 2003). nuf (nuclear fallout) is a maternal effect mutation where the embryos show defects during cellularisation due to reduction of efficient RhoGEF2 recruitment at the furrow, thus affecting F-actin levels at the furrow. Rab11 mutants show a similar phenotype. It has been suggested that the recycling endosome has an important role in the recruitment of RhoGEF2 and therefore in the maintenance of furrow integrity (Cao et al., 2008).

Endocytosis is tightly regulated at the furrow by a number of factors. During early cellularisation, the furrow displays Amphiphysin-positive tubules at the basal region of the furrow (Sokac and Wieschaus, 2008a). Amphiphysin is a protein that can sense and bind to curved endocytic membrane via its conserved BAR domain (for a review, see Daumke et al., 2014). In shibire mutants, the number of tubules is increased, suggesting that these tubules represent intermediate structures formed during endocytic scission, and delay or impairment in endocytosis causes them to become extended (Sokac and Wieschaus, 2008a). As cellularisation progresses, the Amphiphysin-positive tubules are reduced in length and number (Sokac and Wieschaus, 2008a). Overexpression of a cytohesin Arf-GEF Steppke, a positive regulator of endocytosis also causes increase in tubule formation (Lee and Harris, 2013). This indicates that endocytosis at the furrow canal is a highly regulated process and perturbing this balance leads to morphological as well as functional defects in the furrow canal. 


\subsection{Basal closure}

Basal closure during cellularisation involves formation of actin-myosin contractile rings which gradually contract and decrease in diameter, eventually enclosing each nucleus basally. It has been suggested that the timing of basal closure is not dependent on the depth of invagination. Rather, it is regulated in a temporal manner (Royou et al., 2004). bottleneck (bnk) is one of the genes regulating basal closure (Merrill et al., 1988; Schejter and Wieschaus, 1993; Wieschaus and Sweeton, 1988). bnk mutant embryos display premature contraction of actin-myosin network. This causes pinching off of the apical regions of nuclei, which gives the nuclei a bottleneck-like appearance (Schejter and Wieschaus, 1993). Apart from actin and myosin II, other furrow components such as anillin and septin are also required for (Adam et al., 2000; Field et al., 2005). The tyrosine-kinases src64 and tec29 are also required for contraction of microfilaments (Thomas and Wieschaus, 2004).

RhoGEF2 has also been implicated in regulating basal closure. RhoGEF2 mutants show defective Bnk localisation to the furrow canal in late cellularisation and ectopic RhoGEF2 expression leads to recruitment of Bnk (Padash Barmchi et al., 2005). bnk/RhoGEF2 double-mutants show a stronger phenotype compared to RhoGEF2 mutants in the later stages, suggesting their role in a common pathway involved in the regulation of basal closure.

Another pathway of regulation of the furrow canal during late cellularisation is via negative regulation of Rho1 by Steppke. steppke mutants show elevated Rho1 at the furrows which extend perpendicularly and displace the nuclei. The elevation in Rho1 activity is not accompanied by elevated RhoGEF2, suggesting that Sreppke acts independently on Rho1. It is proposed that Steppke keeps the plasma membrane growth in check by acting on a specific sub-population of endocytic events (Lee and Harris, 2013).

\subsection{Aim of the work}

The main focus of this study is the gene slam. The following questions were addressed in this work:-

a) What upstream signals are needed for Slam accumulation at the site of furrow invagination? 
Slam is one of the first markers to reach the furrow canal and as mentioned earlier, is essential for cellularisation. However, it was found that furrow specification takes place even in the absence of Slam. Therefore, the first aim was to determine the origin of signals that are necessary for the specification of the site of invagination that ultimately lead to the accumulation of Slam at the furrow canal.

b) What is the mechanism of Slam localisation and maintenance at the furrow canal?

The second aim was to elaborate on the underlying mechanism behind the localisation of Slam at the furrow canal. Using different approaches, we aimed to determine whether Slam is transported directly/indirectly via microtubules/vesicles. We attempted to learn about its maintenance at the furrow canal by studying the mobility dynamics of Slam protein at the furrow.

c) What is the function of Slam?

Slam is a non-conserved protein with no known functional domains and therefore its biochemical function is yet to be discovered. We have tried to gain some insight into the function of slam by analysing its interactions with other genes such as nullo and spire. Also, Slam protein has been shown to colocalise with its mRNA (Wenzl et al., 2010). The relationship of the protein and the mRNA has been explored further. 


\section{MATERIALS AND METHODS}

\subsection{Materials}

\subsubsection{Reagents}

All standard chemicals were purchased from AppliChem $\mathrm{GmbH}$ (Darmstadt), Gibco BRL (Eggenstein), Invitrogen (Carlsbad, USA), Merck (Darmstadt), Carl Roth GmbH (Karlsruhe) or Sigma-Aldrich (St. Louis, USA) unless otherwise mentioned. RNAsefree water for EMSA was purchased from Ambion.

\subsubsection{Buffers and solutions}

All buffers were prepared according to Sambrook and Russel, 2001 unless otherwise stated.

a) For Immunostaining and western blot

- PBS

$\begin{array}{ll}130 \mathrm{mM} & \mathrm{NaCl} \\ 7 \mathrm{mM} & \mathrm{Na}_{2} \mathrm{HPO}_{4} \\ 3 \mathrm{mM} & \mathrm{NaH}_{2} \mathrm{PO}_{4} \\ \mathrm{pH} & 7.4\end{array}$

- PBST

$0.1 \%$

Tween 20

$1 \mathrm{X}$

PBS

- Embryo fixation solution

$\begin{array}{ll}4.5 \mathrm{ml} & 1 \mathrm{X} \text { PBS } \\ 0.5 \text { or } 1 \mathrm{ml} & \text { Formaldehyde (37\%) } \\ 5 \mathrm{ml} & \text { Heptane }\end{array}$

- Immunostaining blocking buffer

$\begin{array}{ll}1 X & \text { PBS } \\ 5 \% & \text { BSA }\end{array}$

- Western blot blocking buffer

$1 \mathrm{X}$

$5 \%$

$25 \mathrm{mM}$

\section{PBST}

milk powder

- Wet transfer buffer

25mM Tris




$\begin{array}{ll}175 m M & \text { Glycine } \\ 20 \% & \text { Methanol }\end{array}$

b) For mini prep of plasmid DNA

- Solution I

50mM Tris/ $\mathrm{HCl}, \mathrm{pH} 8.0$

$10 \mathrm{mM} \quad$ EDTA

- Solution II

$\begin{array}{ll}1 \% & \text { SDS } \\ 0.2 \mathrm{M} & \mathrm{NaOH}\end{array}$

- Solution III

$3 \mathrm{M}$

Potassium acetate

Adjusted to $\mathrm{pH} 5.4$

with acetic acid

c) For protein purification under native conditions (with GST tag)

- Lysis buffer

- Wash buffer

- Elution buffer

- Storage buffer

All buffers were filtered prior to use

$\begin{array}{ll}50 \mathrm{mM} & \text { Tris/HCl pH } 8.0 \\ 100 \mathrm{mM} & \mathrm{NaCl} \\ 1 \mathrm{mM} & \text { DTT }\end{array}$

50mM Tris/HCl pH 8.0

$500 \mathrm{mM} \quad \mathrm{NaCl}$

$1 \mathrm{mM} \quad$ DTT

$50 \mathrm{mM} \quad$ Tris $/ \mathrm{HCl}$ pH 8.0

$50 \mathrm{mM} \quad \mathrm{NaCl}$

$10 \mathrm{mM} \quad$ Glutathione (freshly added from $10 \mathrm{X}$ stock stored at $-20^{\circ} \mathrm{C}$ )

$1 \mathrm{mM} \quad$ DTT

1X PBS

Glyecerol added to $10 \%$ after buffer exchange 
d) For protein purification under native conditions (with His tag)

- Lysis buffer

$\begin{array}{ll}20 \mathrm{mM} & \mathrm{Na}-\text { Phosphate } \mathrm{pH} 8.0 \\ 500 \mathrm{mM} & \mathrm{NaCl} \\ 20 \mathrm{mM} & \text { Imidazol }\end{array}$

- Wash buffer

$\begin{array}{ll}20 \mathrm{mM} & \text { Na-Phosphate } \mathrm{pH} 8.0 \\ 500 \mathrm{mM} & \mathrm{NaCl} \\ 40 \mathrm{mM} & \text { Imidazol }\end{array}$

- Elution buffer

$\begin{array}{ll}20 \mathrm{mM} & \text { Na-Phosphate } \mathrm{pH} 8.0 \\ 500 \mathrm{mM} & \mathrm{NaCl} \\ 250 \mathrm{mM} & \text { Imidazol }\end{array}$

- Storage buffer

$20 \mathrm{mM} \quad \mathrm{Na}-$ Phosphate $\mathrm{pH} 8.0$
$150 \mathrm{mM} \quad \mathrm{NaCl}$
Glycerol added to $10 \%$ after buffer
exchange

All buffers were filtered prior to use.

e) For protein purification under denaturing conditions (with His tag)

- Lysis buffer

- Buffer A

- Buffer C

$\begin{array}{ll}20 \mathrm{mM} & \mathrm{Na}-\text { Phosphate } \mathrm{pH} 8.0 \\ 500 \mathrm{mM} & \mathrm{NaCl} \\ 20 \mathrm{mM} & \text { Imidazol }\end{array}$

0.1M Na-Phosphate

$10 \mathrm{mM} \quad$ Tris $\mathrm{pH} 8.0(\mathrm{NaOH})$

6M Guanidine hydrochloride

(synthesis grade)

( $\mathrm{pH}$ adjusted prior to use)

$\begin{array}{ll}0.1 \mathrm{M} & \text { Na-Phosphate } \\ 10 \mathrm{mM} & \text { Tris pH } 6.3(\mathrm{HCl}) \\ 8 \mathrm{M} & \text { Urea }\end{array}$


( $\mathrm{pH}$ adjusted prior to use)

- Buffer E

$\begin{array}{ll}0.1 \mathrm{M} & \text { Na-Phosphate } \\ 10 \mathrm{mM} & \text { Tris } \mathrm{pH} 4.5(\mathrm{HCl}) \\ 8 \mathrm{M} & \text { Urea } \\ & \text { (pH adjusted prior to use) }\end{array}$

All buffers were filtered prior to use

f) For protein coupling to $\mathrm{CNBr}$ beads

- Wash buffer for CNBr beads

$1 \mathrm{mM} \quad \mathrm{HCl}$

- Coupling buffer

100mM $\quad \mathrm{NaHCO}_{3} / \mathrm{NaOH} \mathrm{pH} 8.3$

$300 \mathrm{mM} \quad \mathrm{NaCl}$

- Blocking buffer

$0.1 \mathrm{M}$

Tris/HCl pH 8.0

- Wash buffer I

$\begin{array}{ll}0.1 \mathrm{M} & \mathrm{Na}-\text { acetate } \\ 0.5 \mathrm{M} & \mathrm{NaCl} \\ \mathrm{pH} \text { adjusted to } 4.0\end{array}$

- Wash buffer II

$0.1 \mathrm{M}$

Tris/HCl

$0.5 \mathrm{M}$

$\mathrm{NaCl}$

$\mathrm{pH}$ adjusted to 8.0

g) For affinity purification of antibodies

- Wash buffer

$\begin{array}{ll}1 \mathrm{X} & \mathrm{PBS} \\ 300 \mathrm{mM} & \mathrm{NaCl}\end{array}$

- Elution buffer
50mM Glycine
$\mathrm{pH}$ adjusted to 2.5

- Neutralisation buffer

$1 \mathrm{M}$

Tris/HCl pH 11.0

- $20 \%$ Sodium Azide $\left(\mathrm{NaN}_{3}\right)$ 


\section{h) For immunoprecipitation}

- RIPA buffer

$\begin{array}{ll}10 \mathrm{mM} & \text { Tris/HCl pH } 7.5 \\ 150 \mathrm{mM} & \mathrm{NaCl} \\ 0.1 \% & \text { SDS } \\ 1 \% & \text { TritonX } 100 \\ 1 \% & \text { Deoxycholate } \\ 5 \mathrm{mM} & \text { EDTA } \\ 2 \mathrm{mM} & \text { PMSF (freshly added) } \\ 1 \mathrm{X} & \text { Roche protease inhibitor } \\ & \text { cocktail (freshly added) }\end{array}$

i) For fractionation

- Lysis (and wash) buffer

$\begin{array}{ll}50 \mathrm{mM} & \text { Tris } \mathrm{pH} 7.5 \\ 75 \mathrm{mM} & \mathrm{NaCl} \\ 1 \mathrm{mM} & \mathrm{MgCl}_{2} \\ 0.05 \% & \mathrm{NP}-40 \\ 1 \mathrm{mM} & \text { DTT } \\ 2 \mathrm{mM} & \text { PMSF (freshly added) } \\ 1 \mathrm{X} & \text { Roche Protease inhibitor } \\ & \text { cocktail (freshly added) } \\ 0.01 \mathrm{U} / \mu \mathrm{l} & \text { Rnase inhibitor }\end{array}$

- High salt buffer

$1 \mathrm{M} \mathrm{NaCl}$ in Lysis buffer

- 6X Lämmli buffer

$\begin{array}{ll}375 \mathrm{mM} & \text { Tris } \mathrm{HCl} \\ 10 \% & \text { SDS } \\ 50 \% & \text { Glycerol } \\ 0.6 \mathrm{M} & \text { DTT } \\ 0.06 \% & \text { Bromophenol blue }\end{array}$

\section{i) For EMSA}

- 5X EMSA binding buffer

$\begin{array}{ll}5 \mathrm{mg} / \mathrm{ml} & \text { Heparin } \\ 1 \% & \text { Glycerol } \\ 50 \mathrm{mM} & \mathrm{KCl} \\ 10 \mathrm{mM} & \text { DTT } \\ 5.2 \mathrm{mM} & \text { HEPES } \mathrm{pH} 7.0\end{array}$


- EMSA loading dye

$\begin{array}{ll}50 \mathrm{mM} & \text { Tris/Cl pH } 7.5 \\ 50 \% & \text { Glycerol } \\ 0.01 \% & \text { Bromophenol blue }\end{array}$

k) For in-situ hybridisation of embryos

- NTP + Dig labelling mix (10X)

$\begin{array}{ll}10 \mathrm{mM} & \text { ATP } \\ 10 \mathrm{mM} & \text { GTP } \\ 10 \mathrm{mM} & \text { CTP } \\ 6.5 \mathrm{mM} & \text { UTP } \\ 3.5 \mathrm{mM} & \text { Dig-11-UTP, pH 7.5 }\end{array}$

- Hybridisation solution

\begin{tabular}{ll}
$50 \%$ & Formamide \\
$5 \mathrm{X}$ & SSC \\
$50 \mu \mathrm{g} / \mathrm{ml}$ & Heparin \\
$0.2 \%$ & Tween \\
$100 \mu \mathrm{g} / \mathrm{ml}$ & tRNA (For pre-hybridisa \\
& \multicolumn{1}{c}{-tion steps) } \\
DEPC $\mathrm{H}_{2} \mathrm{O}$ & Make upto $50 \mathrm{ml}$ \\
Stored at $-20^{\circ} \mathrm{C}$
\end{tabular}

- DEPC-treated PBST

Recipe of PBST as in immunostaining

\section{l) Other solutions/buffers}

- Cycloheximide buffer

$\begin{array}{ll}0.1 \mathrm{M} & \mathrm{Na} \text {-phosphate } \\ 5 \mathrm{mM} & \mathrm{KCl}\end{array}$

\subsubsection{Media for bacterial culture}

- $\quad$ Luria-Bertani Broth (LB Broth) 


$\begin{array}{cl}5 \mathrm{~g} & \text { Yeast extract } \\ 10 \mathrm{~g} & \mathrm{NaCl} \\ 1000 \mathrm{ml} & \mathrm{ddH}_{2} \mathrm{O} \\ & \\ 10 \mathrm{~g} & \text { Bactotryptone } \\ 5 \mathrm{~g} & \text { Yeast extract } \\ 10 \mathrm{~g} & \mathrm{NaCl} \\ 15 \mathrm{~g} & \mathrm{Agar} \\ 1000 \mathrm{ml} & \mathrm{ddH}_{2} \mathrm{O}\end{array}$

Autoclaved, cooled down to $55^{\circ} \mathrm{C}$ and required antibiotic added, plated into petri-dishes and stored at $4^{\circ} \mathrm{C}$

\subsubsection{Media for flies}

- Fly food

$128 \mathrm{~g}$ thread agar was added to $10 \mathrm{I}$ water and cooked for $2 \mathrm{~h}$ until the agar was completely dissolved. $400 \mathrm{~g}$ fresh baker's yeast, $160 \mathrm{~g}$ soya-bean meal and $1.28 \mathrm{~kg}$ maize meal was added to $4 \mathrm{I}$ water, mixed, and added to the agar and cooked for another 2 h. $1.28 \mathrm{~kg}$ malt extract and $350 \mathrm{~g}$ sugar-beet molasses was added to 21 water and added to the mixture. The food was cooked for $30 \mathrm{~min}$ and allowed to cool down below $60^{\circ} \mathrm{C}$. $24 \mathrm{~g}$ Nipagin dissolved in ethanol and $150 \mathrm{ml}$ propionic acid was added and mixed. The fly food was filled into vials. After solidification of the food, the vials were plugged and stored at $18^{\circ} \mathrm{C}$.

- Apple juice plates

$100 \mathrm{~g}$ sugar was dissolved into $1 \mathrm{I}$ apple juice and kept in a $60^{\circ} \mathrm{C}$ water-bath. $40 \mathrm{ml}$ Nipagin solution (15\% Nipagin in ethanol) was added to the apple juice. $70 \mathrm{~g}$ agar was dissolved into 3 I water and to this, the apple juice mixture was added, mixed and allowed to cool down to $60^{\circ} \mathrm{C}$. The apple juice agar was poured into Petri dishes and stored at $4{ }^{\circ} \mathrm{C}$. 


\subsubsection{Enzymes and Kits}

All restriction enzymes were obtained either from Fermentas (St. Leon-Rot) or New England Biolabs (Ipswich, USA) and used according to the manufacturer's instructions. Additionally, the following enzymes were used:-

- Taq Polymerase (expressed and purified in the lab)

- Pfu DNA Polymerase (expressed and purified in the lab)

- $\quad$ T7 RNA Polymerase (expressed and purified in the lab)

- Transcriptor Reverse transcriptase (Roche)

- Dnasel (Roche)

Following kits were used according to the manufacturer's instructions:-

- MiniElute Gel extraction Kit

- Plasmid Midi Kit Nucleobond AX

- In-fusion HD cloning kit

- RNeasy Micro kit (for RNA purification)

- Individual Cy3 tyramide reagent pack
Qiagen, Hilden

Macherey-Nagel

Clontech

Qiagen, Hilden

PerkinElmer

\subsubsection{Chromatography}

- GST columns (GSTrap HP)

- CNBr activated Sepharose 4B

- Ni-sepharose beads

- PD-10 desalting columns

- Dynabeads protein A(paramagnetic beads)

- Dynabeads MyOne Streptavidin T1 beads
GE Healthcare Life Sciences

GE Healthcare Life Sciences Amersham Pharmacia Biotech

GE Healthcare Life Sciences

Life technologies

Life technologies

\subsubsection{Oligonucleotides used in the study}

All oligonucleotides used in this study were ordered from Eurofins genomics. The nucleotides in red show the changed nucleotide in site-directed mutagenesis. 
Table 2.1 Oligonucleotides used in the study

\begin{tabular}{|c|c|c|}
\hline \multicolumn{3}{|c|}{ Rab11 cloning } \\
\hline $\begin{array}{l}\text { Serial } \\
\text { Nr. }\end{array}$ & Seqeunce $\left(5^{\prime}-3^{\prime}\right)$ & Description \\
\hline SG1 & $\begin{array}{l}\text { CTCCGGTGTTGGCAAAAATAATTT } \\
\text { GCTCTCACG }\end{array}$ & $\begin{array}{l}\text { Forward primer for site-directed } \\
\text { mutagenesis of the } 25^{\text {th }} \text { amino acid of } \\
\text { Rab11 from } \mathrm{S} \text { to } \mathrm{N}\end{array}$ \\
\hline SG2 & $\begin{array}{l}\text { CAAATTATTTTTGCCAACACCGGA } \\
\text { GTCACCGATAAG }\end{array}$ & $\begin{array}{l}\text { Reverse primer for site-directed } \\
\text { mutagenesis of the } 25^{\text {th }} \text { amino acid of } \\
\text { Rab11 from } \mathrm{S} \text { to } \mathrm{N}\end{array}$ \\
\hline SG3 & $\begin{array}{l}\text { TGGATCCCCGGAATTCATGGGTG } \\
\text { CAAGAGAAGACGAG }\end{array}$ & $\begin{array}{l}\text { Forward primer for in-fusion cloning of } \\
\text { Rab11 and Rab11S25N into PGEX-4T1 }\end{array}$ \\
\hline SG4 & $\begin{array}{l}\text { GTCGACCCGGGAATTCTCACTGAC } \\
\text { AGCACTGTTTGCG }\end{array}$ & $\begin{array}{l}\text { Reverse primer for in-fusion cloning of } \\
\text { Rab11 and Rab11S25N into PGEX-4T1 }\end{array}$ \\
\hline \multicolumn{3}{|c|}{ Sequencing } \\
\hline CW20a & ACCCAATGTGCCTGGATGC & $\begin{array}{l}\text { Forward sequencing primer for PGEX- } \\
4 \mathrm{~T} 1\end{array}$ \\
\hline CW20b & CGGGAGCTGCATGTGTCAGA & $\begin{array}{l}\text { Reverse sequencing primer for PGEX- } \\
4 \mathrm{~T} 1\end{array}$ \\
\hline \multicolumn{3}{|c|}{ qRTPCR } \\
\hline SY94 & $\begin{array}{l}\text { GCGAGATCTACCACGCTTTTTCGC } \\
\text { GGTC }\end{array}$ & $\begin{array}{l}\text { Forward primer spanning exon-exon } \\
\text { junction in the } 5 \text { 'UTR of slam }\end{array}$ \\
\hline SY95 & $\begin{array}{l}\text { CGTGGATCCTTTGCTAATAGCTTA } \\
\text { TATACAATG }\end{array}$ & $\begin{array}{l}\text { Reverse primer for detection of slam } \\
\text { mRNA }\end{array}$ \\
\hline SA12 & GTTAAATCGAACAAAAAGCTTAC & $\begin{array}{l}\text { Forward primer spanning exon-exon } \\
\text { junction of first intron of actin }\end{array}$ \\
\hline SA13 & GTGAGGATACCACGCTTGC & $\begin{array}{l}\text { Reverse primer for detection of actin } \\
\text { mRNA }\end{array}$ \\
\hline
\end{tabular}

\subsubsection{Plasmid constructs used in the study}

Table 2.2 Plasmids used in the study

\begin{tabular}{|l|l|l|}
\hline \multicolumn{1}{|c|}{ Name } & \multicolumn{1}{c|}{ Description } & \multicolumn{1}{c|}{ Source } \\
\hline pGEX6OH & GST-His fusion protein & Prof. Jörg Großhans \\
\hline pBS (SK-)-rab11 (EST) & Rab11 cDNA & Bloomington GM06568 \\
\hline
\end{tabular}




\begin{tabular}{|l|l|l|}
\hline pGEX-4T1-rab11 & $\begin{array}{l}\text { N-terminal GST tag fused with } \\
\text { Rab11 protein }\end{array}$ & $\begin{array}{l}\text { Stephanie Gröning } \\
\text { (Pelissier et al., 2003) }\end{array}$ \\
\hline pGEX-4T1-rab11S25N & $\begin{array}{l}\text { N-terminal GST tag fused with } \\
\text { Rab11 protein with a point mutation } \\
\text { changing the 25th amino acid from } \\
\text { Serine to Asparagine }\end{array}$ & $\begin{array}{l}\text { Stephanie Gröning } \\
\text { (Pelissier et al., 2003) }\end{array}$ \\
\hline pProEXHTb-p150-KIND & $\begin{array}{l}\text { p150-KIND with an N-terminal his- } \\
\text { tag Prof. E. Kerkhoff }\end{array}$ & Prom \\
\hline pQEslam $\Delta$ C651 & $\begin{array}{l}\text { Slam N-terminal fragment from } \\
\text { amino acid 1 to 545 fused with a 6X } \\
\text { His tag at the C-terminal }\end{array}$ & Prof. Jörg Großhans \\
\hline pCS2GFP & GFP sequence under SP6 promoter & Dr. Shuling Yan \\
\hline pCS2slam2-1(slam20) & $\begin{array}{l}\text { N-terminal GFP sequence fused } \\
\text { with slam sequence coding from } \\
\text { amino acid 200 to 330 }\end{array}$ & Dr. Shuling Yan \\
\hline pCS2slam2-2(slam21) & $\begin{array}{l}\text { N-terminal GFP sequence fused } \\
\text { with slam sequence coding from } \\
\text { amino acid 331 to 476 }\end{array}$ & Dr. Shuling Yan \\
\hline
\end{tabular}

\subsubsection{Primary antibodies}

Table 2.3 Antibodies used in the study

\begin{tabular}{|c|c|c|c|c|}
\hline \multirow[t]{2}{*}{ Antibody } & \multirow{2}{*}{$\begin{array}{l}\text { Raised } \\
\text { in }\end{array}$} & \multicolumn{2}{|c|}{ Dilution and working concentration } & \multirow[b]{2}{*}{ Source } \\
\hline & & Staining & Western & \\
\hline B-Gal & Mouse & $1: 5000(0.2 \mu \mathrm{g} / \mathrm{ml})$ & - & Roche \\
\hline Dia & Rabbit & $1: 1000$ & - & $\mathrm{ZMBH}$ \\
\hline Dlg & Mouse & $1: 100(\sim 0.4 \mu \mathrm{g} / \mathrm{ml})$ & - & Hybridoma bank 4F3 \\
\hline Nullo & Mouse & $1: 10(\sim 0.3 \mu \mathrm{g} / \mathrm{ml})$ & - & Hybridoma bank $5 \mathrm{C} 3-12$ \\
\hline Rab11 & Rabbit & $1: 1000$ & - & Prof. D.F. Ready \\
\hline Slam & Rabbit & $1: 5000(\sim 1 \mu \mathrm{g} / \mathrm{ml})$ & $1: 5000(\sim 1.1 \mu \mathrm{g} / \mathrm{ml})$ & Prof. Jörg Großhans \\
\hline Slam & $\begin{array}{l}\text { Guinea } \\
\text { pig }\end{array}$ & $1: 5000$ & $1: 5000$ & Prof. Jörg Großhans \\
\hline $\begin{array}{l}\text { Slam (affinity } \\
\text { purified) }\end{array}$ & Rabbit & - & $1: 5000(\sim 1.2 \mu \mathrm{g} / \mathrm{ml})$ & Purified by me \\
\hline $\begin{array}{l}\text { Spire (affinity } \\
\text { purified) }\end{array}$ & $\begin{array}{l}\text { Guinuea } \\
\text { pig }\end{array}$ & $1: 1000(\sim 10 \mu \mathrm{g} / \mathrm{ml})$ & $1: 2000(\sim 5 \mu \mathrm{g} / \mathrm{ml})$ & Purified by me \\
\hline
\end{tabular}




\subsubsection{Secondary antibodies/dyes}

Alexa-conjugated secondary antibodies for immunostaining were purchased from life technologies (Invitrogen) and used at a dilution of 1:500 $(4 \mu \mathrm{g} / \mathrm{ml})$. DAPI for staining of DNA was used at a working concentration $(0.2 \mu \mathrm{g} / \mathrm{ml})$. Anti-Digoxigenin-peroxidase antibodies were purchased from Roche and used at a dilution of 1:200 $(0.75 \mathrm{U} / \mathrm{ml})$. For western blots, IRDye-800CW and IRDye-680 secondary antibodies were purchased from LI-COR Biotechnology and used at a dilution of 1:20000 $(0.05 \mu \mathrm{g} / \mathrm{ml})$.

\subsubsection{Bacterial cell lines}

Following strains of E.coli were used:

- DH5 $\alpha$ for plasmid amplification:

$\mathrm{F}^{-}, \varnothing 80 \mathrm{~d} / a c Z \Delta \mathrm{M} 15, \triangle$ (lacZYA-argF)U169, deoR, recA1, endA1, hsdR17(rK-, $\left.\mathrm{mK}^{+}\right)$, phoA, supE44, $\lambda-$,thi-1, gyrA96, relA1

- BL21DE was used for protein expression:

$\mathrm{F}^{-}, d c m$, omp $T, h s d \mathrm{~S}\left(r_{\mathrm{B}}^{-} m_{B^{-}}\right)$, gal l(DE3).

\subsubsection{Fly stocks}

All the fly stocks were obtained from the Bloomington stock centre, unless otherwise stated.

Table 2.4 Fly stocks used in the study

\begin{tabular}{|c|c|c|}
\hline Stock name & Stock genotype & $\begin{array}{c}\text { Source/Lab serial } \\
\text { nr. }\end{array}$ \\
\hline oregon- $R$ & $+/+$ & A401 \\
\hline $\begin{array}{l}\text { slam5'rescue/CyO; } \\
\text { GFPslam/TM3 }\end{array}$ & $\begin{array}{l}\text { w ; Df(2L)slam }\{w+\} \text { Frt[2L] }\{\text { neoR }\} \text { c } \\
\text { slam5' }\{w+\}[58] / \text { CyO ; GFPslam }\{w+\} / T M 3, \text { Sb }\end{array}$ & Prof. J. Großhans \\
\hline $\begin{array}{l}\text { slam3'rescue/CyO; } \\
\text { Dr/TM3 }\end{array}$ & $\begin{array}{l}\text { w ; Df(2L)slam }\{w+\} \text { slam3' }\{w+\} \text { Frt[2L }]\{n e o R\} / \\
\text { CyO ; Dr/TM3, Sb }\end{array}$ & Prof. J. Großhans \\
\hline slam-9.5 & slam[9.35] b pr Frt[2L]\{neoR\} / CyO & H093 \\
\hline
\end{tabular}




\begin{tabular}{|c|c|c|}
\hline slam-B4.1 & slam[B4.1] b pr Frt[2L]\{neoR\} / CyO & H094 \\
\hline Flp122; ovoD2L & hs-Flp[122]; ovoDFrt2L[40A]/If/CyO, hs-hid & $\begin{array}{l}\text { Maintained in the } \\
\text { lab }\end{array}$ \\
\hline mat-GFPslam & $\begin{array}{l}\text { w; tub-Gal4-VP16[67] }\{w+\} ; \text { UASp-GFP- } \\
\text { slam[1]\{w+\} }\end{array}$ & $\mathrm{H} 016$ \\
\hline GFPslam & w ; GFPslam[68] $\{$ w+ $\}$ & $\mathrm{H} 087$ \\
\hline SAS6GFP/TM6c & w ; ubi-GFP-SAS6 $\{w+\} /$ TM6c, Sb & Prof. J. Raff \\
\hline $\begin{array}{l}\text { Sp/CyO; GFP- } \\
\text { SAS6/TM6c }\end{array}$ & w ; Sp/CyO; ubi-GFP-SAS6 $\{w+\} /$ TM6c, Sb & Zhiyi Lv \\
\hline shibire & w shi[1] / FM6, y B & A119 \\
\hline shibire; Sp/CyO & w shi[1] / FM7c, y B; Sp/CyO & Zhiyi Lv \\
\hline rab11YFP-CHR & rab11YFP-wt[CHR-7]/TM3, Sb & $\mathrm{H} 008$ \\
\hline GFPslam; rab11YFP & $\begin{array}{l}\text { w; tub-Gal4-VP16[67]\{w+\} UASp-GFP- } \\
\text { slam }\{w+\} / C y O ; \text { rab11YFP-wt[CHR-7]/TM3, Sb }\end{array}$ & Prof. J. Großhans \\
\hline nuf/TM6B & nuf[1] sr e ca / TM6B, Tb Hu & $\mathrm{H} 010$ \\
\hline Sp/CyO; nuf/TM3 & Sp/CyO; nuf[1] sr e ca/ TM3, Sb & Prof. J. Großhans \\
\hline eos-slam & w; UASp-meos2-slam $\{$ w+ $\}$ & Dr. P. Laupsien \\
\hline mat67.15 & $\begin{array}{l}\text { tub-Gal4-VP16[67]\{w+\}; tub-Gal4- } \\
\text { VP16[15]\{w+\} }\end{array}$ & $\begin{array}{l}\text { Prof. D. St. } \\
\text { Johnston, UK }\end{array}$ \\
\hline his-RFP & w; histone2Av-mRFP[1]\{w+\} & B384 \\
\hline nullo/FM7; If/CyO & Df(1)nullo[6F12] / FM7; If/CyO & $\begin{array}{l}\text { (Hunter and } \\
\text { Wieschaus, 2000) }\end{array}$ \\
\hline Dfslam/hb-lacz & Df(2L)BSC5/CyO, hb-lacZ & Prof. J. Großhans \\
\hline spir $^{1}$ & spir[1]cn[1]bw[1]/CyO, I(2)DTS513[1] & $\mathrm{H} 064$ \\
\hline Dfspire & Df(2L)Exel ${ }^{6046}$ & Bloomington \\
\hline \multicolumn{2}{|c|}{ Following alleles of spire were obtained from Tübingen stock centre } & Tübingen Stock nr. \\
\hline spir $2 \mathrm{~L}-62-29$ & $\begin{array}{l}\text { w (P[hs-Flp]122); al? dp? b? pr P[FRT, } \\
\text { neoR]40A/CyO, P[hs-hid, w+] }\end{array}$ & L210 \\
\hline spir'2L-75-28 & $\begin{array}{l}\text { w (P[hs-Flp]122); al? dp b? pr P[FRT, } \\
\text { neoR]40A/CyO, P[hs-hid, w+] }\end{array}$ & L214 \\
\hline spir2L-133-31 & $\begin{array}{l}\text { w (P[hs-Flp]122); al? dp? b? pr P[FRT, } \\
\text { neoR]40A/CyO, P[hs-hid, w+] }\end{array}$ & L228 \\
\hline spir2L-146-30 & $\begin{array}{l}\text { w (P[hs-Flp]122); al dp b pr P[FRT, } \\
\text { neoR]40A/CyO, P[hs-hid, w+] }\end{array}$ & L235 \\
\hline spir $2 \mathrm{~L}-210-2(\mathrm{ts})$ & $\begin{array}{l}\text { w (P[hs-Flp]122); al? dp b pr P[FRT, } \\
\text { neoR]40A/CyO, P[hs-hid, w+] }\end{array}$ & L253 \\
\hline
\end{tabular}




\begin{tabular}{|c|c|c|}
\hline spir2L-216-18 & $\begin{array}{l}\text { w (P[hs-Flp]122); al? dp? b? pr? P[FRT, } \\
\text { neoR]40A/CyO, P[hs-hid, w+] }\end{array}$ & L258 \\
\hline spir2L-244-35 & $\begin{array}{l}\text { w (P[hs-Flp]122); al? dp b? pr P[FRT, } \\
\text { neoR]40A/CyO, P[hs-hid, w+] }\end{array}$ & L265 \\
\hline \multicolumn{3}{|c|}{ Generated in this work: } \\
\hline gfpslam; nuf & $\begin{array}{l}\text { tub-Gal4-VP16[67] }\{w+\} ; \text { UASp-GFP- } \\
\text { slam }\{w+\} / \text { CyO; nuf[1] sr e ca /TM3 }\end{array}$ & - \\
\hline shi; gfpslam & $\begin{array}{l}\text { w shi[1] / FM7c, y B; tub-Gal4-VP16[67] }\{w+\} \\
\text { UASp-GFP- slam }\{w+\} / \text { CyO }\end{array}$ & - \\
\hline $\begin{array}{l}\text { nullo/FM7; } \\
\text { Dfslam5'/CyO }\end{array}$ & $\begin{array}{l}\text { Df(1)nullo[6F12] / FM7 ; Df(2L)slam }\{w+\} \\
\text { Frt[2L]\{neoR }\} \text { c slam5' }\{w+\} / C y O\end{array}$ & - \\
\hline
\end{tabular}

\subsubsection{Microscopy}

- Zeiss Stemi 2000

- Leica MZ125

- Microinjection microscope

- $\quad$ LSM 780

- Zeiss Axiovert 200 M Ultra-view spinning Disc confocal microscope

- Zeiss Axioplan 2 Fluorescence microscope
Carl Zeiss

Leica

Carl Zeiss

Carl Zeiss

Carl Zeiss

Carl Zeiss

\subsubsection{Other materials}

- Ribolock RNAse inhibitor

- Cy3-UTP

- Cycloheximide

- Complete Mini (EDTA-free) Protease Inhibitor Cocktail

- Aquapolymount

- Coverslips

- Glass slides

- Fly vials

- Glass pipettes $(25 \mathrm{ml}, 20 \mathrm{ml}, 10 \mathrm{ml}, 5 \mathrm{ml})$

- Pasteur pipettes

- Glass homogenizer
Thermo Scientific

Perkin Elmer

Sigma

Roche

Polysciences, Inc.

Menzel

Thermo Scientific

Greiner

Silber Brandt

Brandt

B. Braun Biotech International 
- Dynamag - spin magnet

- Petri dishes

- Pipet-aid

- Micropipettes $(1000 \mu \mathrm{l}, 200 \mu \mathrm{l}, 20 \mu \mathrm{l}, 2 \mu \mathrm{l})$

- Micropipette tips (1000 $\mu \mathrm{l}, 200 \mu \mathrm{l}, 2 \mu \mathrm{l})$

- Safe-seal RNase-free tips $1000 \mu \mathrm{l}, 200 \mu \mathrm{l}$, $20 \mu \mathrm{l}, 10 \mu \mathrm{l})$

- Falcon tubes $(50 \mathrm{ml}, 15 \mathrm{ml})$

- Eppendorf tubes (1.5ml, 2ml, $5 \mathrm{ml})$

- PCR tubes

- $\quad$ 10S and 3S VoltaLef Halocarbon oil
Life technologies

Greiner

Drummond

Gilson

Eppendorf

Biozym

BD Falcon

Eppendorf

Brand, Wertheim

Lehmann \& Voss \& Co.

\subsubsection{Other equipment}

- UGA-40 MLC ablation system with DPSL-355/14 laser

Rapp Optoelectronic

- Äkta pure GE Healthcare Life Sciences

- Odyssey CLx Infrared imaging system LI-COR Biosciences

- CFX96 Real time PCR detection system/ C1000 touch thermal cycler

Biorad

- Typhoon variable mode imager

Amersham

\subsubsection{Softwares}

- Adobe Photoshop CS6

Adobe

- Adobe Illustrator CS6

Adobe

- FIJI

$\mathrm{NIH}$

- Zen 2012

Carl Zeiss

- Lasergene

GATC biotech

- Microsoft excel

Microsoft

- Microsoft word

Microsoft

- Zotero

Roy Rosenzweig Center for History and New Media 


\subsection{Methods}

\subsubsection{DNA methods}

\subsubsection{Molecular cloning}

All the molecular cloning methods were carried out according to Sambrook and Russel, 2001, unless otherwise stated.

\subsubsection{Polymerase chain reaction (PCR)}

PCR reactions were carried out using Taq or Pfu DNA polymerase. Standard PCR reactions were carried out with the following reagents in the mentioned concentrations: 50-200 ng DNA template, $0.5 \mu \mathrm{M}$ forward and reverse primers, $50 \mu \mathrm{M}$ dNTP (each), 1X PCR buffer (depending on the polymerase), 1-2 units (per $50 \mu$ l of reaction) Taq or Pfu polymerase.

Following conditions were used for a general reaction:

Step 1 (Initial denaturation): $\quad 95^{\circ} \mathrm{C} \quad-\quad 2 \min$

Step 2 (Denaturation): $\quad 95^{\circ} \mathrm{C} \quad-\quad 30 \mathrm{sec}$

Step 3 (Annealing): $\quad 50-60^{\circ} \mathrm{C} \quad-\quad 1 \mathrm{~min}$

(depending on the annealing temperatures of the primers)

Step 4 (Elongation): $\quad \quad 72^{\circ} \mathrm{C} \quad-\quad 1 \mathrm{~min} / \mathrm{Kb}$ to be amplified

Step 5 (Repetition of cycles) Steps 2 to $4 \quad-\quad 30$ cycles

Step 6 (Final elongation) $\quad 72^{\circ} \mathrm{C} \quad-\quad 7 \mathrm{~min}$

Step 7 (Hold) $\quad 4^{\circ} \mathrm{C} \quad-\infty$

\subsubsection{Site-directed mutagenesis}

Site-directed mutagenesis was carried out to change the $25^{\text {th }}$ amino acid in Rab11 protein from Serine to Asparagine (AGT to AAT). The following PCR program was used: 


\begin{tabular}{lcll}
\hline Step 1 (Initial denaturation): & $95^{\circ} \mathrm{C}$ & - & $5 \mathrm{~min}$ \\
Step 2 (Denaturation): & $95^{\circ} \mathrm{C}$ & - & $50 \mathrm{sec}$ \\
Step 3 (Annealing): & $62^{\circ} \mathrm{C}$ & - & $50 \mathrm{sec}$ \\
Step 4 (Elongation): & $68^{\circ} \mathrm{C}$ & - & $5.5 \mathrm{~min}$ \\
Step 5 (Repetition of cycles) Steps 2 to 4 & - & 18 cycles \\
Step 6 (Final elongation) & $68^{\circ} \mathrm{C}$ & - & 7 min \\
Step 7 (Hold) & $4^{\circ} \mathrm{C}$ & - & $\infty$
\end{tabular}

\subsubsection{In-fusion cloning}

In-fusion cloning was carried out according to the manufacturer's instructions.

\subsubsection{Purification of DNA/RNA by phenol-chloroform extraction}

DNA/RNA sample was made up to $200 \mu \mathrm{l}$ by adding $\mathrm{ddH}_{2} \mathrm{O} / \mathrm{DEPC}-\mathrm{H}_{2} \mathrm{O}$. To this, $200 \mu \mathrm{l}$ of Phenol:Chloroform:Isoamylalcohol was added and mixed well. The sample was centrifuged at 13,000 rpm for $5 \mathrm{~min}$. The upper phase was transferred to a new eppendorf. $200 \mu$ of Chloroform was added to this and mixed well. The sample was centrifuged again at 13,000 rpm for $5 \mathrm{~min}$. The upper phase was again transferred to a fresh eppendorf. To this, $20 \mu \mathrm{l}$ of $3 \mathrm{M} \mathrm{NaAc}$ and $500 \mu \mathrm{l}$ Ethanol was added and mixed. The sample was kept at $-20^{\circ} \mathrm{C}$ overnight (or $-80^{\circ} \mathrm{C}$ for 1 hour). Then the sample was pelleted by centrifuging at $14,000 \mathrm{rpm}$ for $30 \mathrm{~min}$ at $4^{\circ} \mathrm{C}$. The supernatant was discarded. $100 \mu \mathrm{l}$ of $70 \%$ Ethanol was added to the pellet. Again the sample was centrigued at 14,000 rpm for $15 \mathrm{~min}$. Supernatant was discarded and pellet dried either in the Speedvac or under the hood. The dried pellet was resuspended in $20 \mu \mathrm{l}$ of $\mathrm{ddH}_{2} \mathrm{O} / \mathrm{DEPC}-\mathrm{H}_{2} \mathrm{O}$.

\subsubsection{DNA sequencing}

DNA sequencing was carried out with the sequencing facility at the Department of Developmental Biochemistry, GZNB, University of Göttingen. 


\subsubsection{In-vitro transcription}

In-vitro transcription was carried out to prepare Cy3-labelled GFP, slam2-1 and slam22 fragments for EMSA. The pCS2-GFP, pCS2-slam2-1 and pCS2-slam2-2 plasmids was restriction digested using Notl and purified using phenol-chloroform extraction (Refer to 2.2.1.5). The linearised plasmids were used as a template for in-vitro transcription. The following components were mixed into an eppendorf for the in-vitro transcription reaction:

\section{Component}

Linearised plasmid

Transcription buffer

rCTP

rGTP

rATP

rUTP

Су3-UTP

DTT

Ribolock RNAse-inhibitor

SP6 polymerase

Pyrophosphatase

$\mathrm{dH}_{2} \mathrm{O}$ (Rnase-free)

Total Volume
Volume

$5 \mu \mathrm{l}$

$2.5 \mu \mathrm{l}$

$1 \mu \mathrm{l}$

$1 \mu \mathrm{l}$

$1 \mu l$

$0.64 \mu \mathrm{l}$

$0.36 \mu l$

$0.75 \mu l$

$1 \mu \mathrm{l}$

$1 \mu \mathrm{l}$

$0.2 \mu \mathrm{l}$

$10.55 \mu \mathrm{l}$

$25 \mu \mathrm{l}$
Stock Concentration

$0.2 \mu \mathrm{g} / \mu \mathrm{l}$

$10 \mathrm{X}$

$10 \mathrm{mM}$

$10 \mathrm{mM}$

$10 \mathrm{mM}$

$10 \mathrm{mM}$

$100 \mathrm{nmol}$

$1 \mathrm{M}$

$40 \mathrm{U} / \mu \mathrm{l}$

$20 \mathrm{U} / \mu \mathrm{l}$

$0.5 \mathrm{U} / \mu \mathrm{l}$

The reaction was kept at $37^{\circ} \mathrm{C}$, overnight. $1 \mu$ of Dnasel (Stock:10 U/ $\mu$ l) was added and kept at $37^{\circ} \mathrm{C}$ for $30 \mathrm{~min}$. The RNA was purified using Qiagen RNeasy kit, eluted in $30 \mu \mathrm{l}$ RNAse-free water and stored at $-20^{\circ} \mathrm{C}$ until further use. 


\subsubsection{Reverse transcription}

$5 \mu \mathrm{l}$ RNA sample (ranging from 0.2 to $1.2 \mu \mathrm{g} / \mu \mathrm{l}$ ) was mixed with $7 \mu \mathrm{l} \mathrm{dH}_{2} \mathrm{O}$ (RNasefree) and $1 \mu \mathrm{loligo}(\mathrm{dT})(100 \mathrm{pmol} / \mu \mathrm{l})$ and kept at $65^{\circ} \mathrm{C}$ for $10 \mathrm{~min}$. After that, the following components were added to the sample (Stock concentration in brackets):

$\begin{array}{lll}\text { 5X RT buffer } & - & 4 \mu \mathrm{l} \\ \text { RNAse inhibitor }(40 \mathrm{U} / \mu \mathrm{l}) & - & 0.5 \mu \mathrm{l} \\ \text { dNTP mix }(10 \mathrm{mM}) & - & 2 \mu \mathrm{l} \\ \text { Reverse transcriptase }(20 \mathrm{U} / \mu \mathrm{l}) & - & 0.5 \mu \mathrm{l}\end{array}$

The sample (total volume $20 \mu \mathrm{l}$ ), was kept at $55^{\circ} \mathrm{C}$ for $30 \mathrm{~min}$ and then at $85^{\circ} \mathrm{C}$ for 10 min. The cDNA was then stored at $-20^{\circ} \mathrm{C}$.

\subsubsection{Quantitative real-time PCR (qRT-PCR)}

The following components were added for each qPCR reaction:

$\begin{array}{lll}\text { SYBr green qPCR mix } & - & 12.5 \mu \mathrm{l} \\ \mathrm{dH}_{2} \mathrm{O} & - & 8 \mu \mathrm{l} \\ \text { Forward primer } & - & 1.25 \mu \mathrm{l} \\ \text { Reverse primer } & - & 1.25 \mu \mathrm{l} \\ \text { CDNA } & - & 2 \mu \mathrm{l}\end{array}$

The following GPCR program was used for detection of slam and actin mRNA:

$\begin{array}{lcll}\text { Step } 1 \text { (Initial denaturation): } & 95^{\circ} \mathrm{C} & - & 3 \mathrm{~min} \\ \text { Step } 2 \text { (Denaturation): } & 95^{\circ} \mathrm{C} & - & 10 \mathrm{sec} \\ \text { Step } 3 \text { (Annealing): } & 60^{\circ} \mathrm{C} & - & 15 \mathrm{sec} \\ \text { Step } 4 \text { (Elongation): } & 72^{\circ} \mathrm{C} & - & 30 \mathrm{sec} \\ \text { Step } 5 \text { (Repetition of cycles) Steps } 2 \text { to } 4 & - & 40 \mathrm{cycles} \\ \text { Step } 6 \text { (Melting curve) } & 55^{\circ} \mathrm{C} \text { to } 95^{\circ} \mathrm{C} & - & 10 \mathrm{sec}+\text { plate read } \\ & \left(\text { Increment of } 0.5^{\circ} \mathrm{C}\right)\end{array}$




\subsubsection{Biochemical methods}

\subsubsection{Protein purification}

Expression of all recombinant proteins was carried out in E.coli. BL21DE

\section{a) Purification of GST-tagged proteins under native conditions:}

GST, GST-Rab11 and GST-Rab11S25N recombinant protein expression was induced with $0.2 \mathrm{mM}$ IPTG at $18^{\circ} \mathrm{C}$ overnight. The cells were harvested by centrifuging at $5000 \mathrm{rpm}$ for $30 \mathrm{~min}$. They were resuspended in lysis buffer for GST-tagged proteins and incubated with $1 \mathrm{mg} / \mathrm{ml}$ lysozyme and a pinch of DNase for $30 \mathrm{~min}$ on ice. The cells were then lysed using a microfluidiser. The soluble part was obtained by centrifuging twice at 12,000 rpm for $20 \mathrm{~min}$ each to remove the insoluble fraction. The supernatant (soluble fraction) was passed through a Glutathione Sepharose prepacked column (GSTrap HP column), washed with approximately $10 \mathrm{ml}$ of wash buffer and eluted with elution buffer. The eluted protein was then exchanged to PBS using a PD10 column and the protein was concentrated to $1 \mathrm{mg} / \mathrm{ml}$ for injection into GFPslam embryos.

\section{b) Purification of His-tagged proteins under native conditions:}

Expression of Slam $\Delta \mathrm{C} 651$ protein was induced with $0.1 \mathrm{~mm}$ IPTG at $18^{\circ} \mathrm{C}$ overnight. The cells were harvested and lysed as described earlier, but in lysis buffer for histagged proteins. The supernatant (soluble fraction) was passed through a NickelSepharose prepacked column (HisTrap HP column), washed with approximately $10 \mathrm{ml}$ of wash buffer and eluted with elution buffer. The eluted protein was then exchanged to storage buffer using a PD10 column and the protein was concentrated to $1.5 \mathrm{mg} / \mathrm{ml}$. Glycerol was added to the protein sample to make the final concentration $10 \%$ glycerol and the protein was snap-frozen in liquid Nitrogen and stored at $-80^{\circ} \mathrm{C}$.

\section{c) Purification of His-tagged proteins under denaturing conditions:}

Expression of p150-KIND was induced with $0.5 \mathrm{mM} \mathrm{IPTG}$ at $37^{\circ} \mathrm{C}$ for three hours. The cells were harvested and lysed as described earlier, but in lysis buffer for his-tagged proteins under denaturing conditions. The supernatant (soluble fraction) was rocked with $5 \mathrm{ml}$ Nickel-Sepharose beads for one hour at room temperature. The suspension was added to a gravity column and washed with approximately $50 \mathrm{ml}$ of wash buffer 
and eluted with elution buffer. The eluted protein was then exchanged to storage buffer using a PD10 column and the protein was concentrated to $1.5 \mathrm{mg} / \mathrm{ml}$. Glycerol was added to the protein sample to make the final concentration $10 \%$ glycerol and the protein was snap-frozen in liquid Nitrogen and stored at $-80^{\circ} \mathrm{C}$, before sending it out to the company for antibody production.

\subsubsection{Affinity purification of antibodies}

\section{a) Preparation of the CNBr-activated Sepharose beads:}

$1 \mathrm{~g}$ of dry CNBr-sepharose beads were swelled in $10 \mathrm{ml}$ of $1 \mathrm{mM} \mathrm{HCl}$ for $15 \mathrm{~min}$. The beads were washed on a sintered glass filter with about $200 \mathrm{ml}$ of $1 \mathrm{mM} \mathrm{HCl}$. Washes were carried out in several aliquots.

\section{b) Coupling of protein to beads:}

The gel was washed with $5 \mathrm{ml}$ coupling buffer. $A_{280}$ of the protein solution was taken. The protein solution was then added to the gel in a 1:2 gel:buffer ratio. The suspension was mixed on a rocker for $3 \mathrm{hr}$ at room temperature. The beads were allowed to settle down and the supernatant removed after taking the $\mathrm{A}_{280}$ reading to confirm binding. Blocking buffer was added to the gel and mixed for $2 \mathrm{hr}$ at room temperature. The suspended beads were poured into a column. The column was washed with five cycles of alternating low and high pH buffers (Buffer I and Buffer II). Then the column was washed with five column volumes of PBS. The column was stored at $4^{\circ} \mathrm{C}$.

\section{c) Loading of serum and elution of antibodies:}

The serum was centrifuged twice at $15,000 \mathrm{rpm}$ for $20 \mathrm{~min}$ each at $4^{\circ} \mathrm{C}$. The cleared serum was then applied to the column at a flow rate of $0.5 \mathrm{mg} / \mathrm{ml}$. The flow-through was collected and saved. The column was washed first with ten column volumes of PBS $+500 \mathrm{mM} \mathrm{NaCl}$ and then with PBS $+1 \mathrm{M} \mathrm{NaCl}$. For elution, $100 \mu$ l of neutralisation buffer was added to the collection tubes and $900 \mu \mathrm{l}$ fractions were taken via elution with low $\mathrm{pH}$ buffer. The peak fractions were identified using $A_{280}$ and pooled together. The pooled sample was then exchanged to PBS using PD-10 columns. The antibody solution was then concentrated $(\sim 5 \mathrm{mg} / \mathrm{ml}$ for Rabbit anti-Slam antibodies and $\sim 10$ $\mathrm{mg} / \mathrm{ml}$ for Guinuea pig anti-KIND antibodies). Na-Azide was added to $0.02 \%$ to the antibodies and they were stored at $4^{\circ} \mathrm{C}$. 


\subsubsection{Western blot}

\section{a) Embryo collection:}

Embryos were staged from 1.5 to 2.5 hours on apple-juice agar plates and dechorionated in 50\% Klorix bleach for 90 seconds. The dechorionated embryos were collected into a weighed eppendorf tube and spun down gently. The weight of the embryos was determined ( $1 \mathrm{mg}=100$ embryos). 1X Lämmlli buffer was added and the embryos were homogenised. The sample was then heated to $95^{\circ} \mathrm{C}$ for $5 \mathrm{~min}$ and centrifuged at 14,000 rpm for $1 \mathrm{~min}$. The embryos were either loaded immediately on an SDS-gel for a western blot or stored in $-20^{\circ} \mathrm{C}$ for future use.

\section{b) Western blot procedure:}

A volume corresponding to 10 embryos was loaded on an SDS-PAGE gel for a general western blot, unless otherwise stated. The gel was run at $16 \mathrm{~mA}$ constant current until the dye front just ran off. The proteins from the gel were transferred onto a nitrocellulose membrane either using a semi-dry transfer for 1 hour (Westerns for Slam and $\alpha$-tubulin) or wet transfer for overnight (Westerns for GFP-slam and Spire). The membrane was then blocked in 5\% milk powder in PBT for 35 min and incubated with primary antibody either overnight at $4^{\circ} \mathrm{C}$ or 2 hours at room temperature. The membrane was rinsed thrice in PBT and given four 10 minute-PBT washes. The membrane was incubated with secondary antibody for 1 and a half hours at room temperature, protected from light. Then it was again rinsed thrice in PBT and given four 10 minute-PBT washes, after which the bands were detected using the Odyssey CLx Infrared Imaging system.

\subsubsection{EMSA}

Six serial dilutions of the protein were carried out in protein storage buffer. The Cy3labelled RNA was heated to $95^{\circ} \mathrm{C}$ for $1 \mathrm{~min}$ and then allowed to cool to $25^{\circ} \mathrm{C}$ slowly on a thermomixer. A mastermix for twelve samples was prepared with the following components:

Component

RNase-free water
Volume per sample

$12 \mu \mathrm{l}$ 
Yeast RNA $(10 \mu \mathrm{g} / \mu \mathrm{l})$

$5 \mathrm{X}$ binding buffer

Cy3-labelled RNA ( 200 ng/ $/$ l)
$1 \mu l$

$4 \mu \mathrm{l}$

$1 \mu \mathrm{l}$

The above mastermix was aliquoted into 12 eppendorfs ( $18 \mu$ l each). To each reaction tube, $3 \mu \mathrm{l}$ of protein dilution was added. Storage buffer without any protein was used as a negative control. The reaction tubes were mixed and kept at room temperature for $30 \mathrm{~min}$ for binding. $5 \mu$ l of EMSA loading dye was added to samples and mixed. The samples were loaded on $1 \%$ Agarose gels and run at $40 \mathrm{~V}$ constant voltage until the dye front ran three-fourths of the gel length. The RNA bands were visualised using Typhoon phosphoimager.

\subsubsection{Immunoprecipitation}

Staged wild-type embryos were collected by dechorionating the embryos (Refer to 2.2.2.3 a). After collecting the embryos in an eppendorf, spinning them down and weighing, they were snap-frozen in liquid nitrogen and stored at $-80^{\circ} \mathrm{C} .40 \mu \mathrm{l}$ dynabeads protein A magnetic beads were washed twice with $1 \mathrm{ml}$ PBS each and incubated with rabbit slam antibody for 1 hour. After one hour, the beads were washed thrice with PBS and kept on ice. Approximately $100 \mathrm{mg}$ of embryos ( 10,000 embryos) were lysed in $1 \mathrm{ml}$ RIPA buffer using a dounce homogeniser and transferred into a cool eppendorf using a pre-cooled pasteur pipette. The lysate was centrifuged at 14,000 rpm at $4^{\circ} \mathrm{C}$ for $15 \mathrm{~min}$. The lipid layer floating on the top was sucked off using an aspirator pump. Input sample was taken such that the final concentration in 1X Lämmlli buffer was $1 \mathrm{embryo} / \mu \mathrm{l}$. The sample was boiled at $95^{\circ} \mathrm{C}$ immediately for $5 \mathrm{~min}$. The rest of the lysate was added to the antibody-conjugated dynabeads and rotated on a wheel for 2 hours at $4^{\circ} \mathrm{C}$. Subsequently, the beads were settled down using Dynamag spin-magnetic and an unbound sample (supernatant) was taken in the same way as input sample. The beads were washed thrice with RIPA buffer and subsequently, protein was eluted from the beads such that the final concentration in 1X Lämmlli buffer was 50 embryos $/ \mu$ l. Sample was heated immediately at $95^{\circ} \mathrm{C}$ for $5 \mathrm{~min}$. The input ( 10 embryos), unbound ( $\sim 10$ embryos) and bound ( $\sim 500$ embryos) samples were loaded onto an $8 \%$ SDS-PAGE gel to carry out a western blot. 


\subsubsection{Pulldown of GFPslam using paramagnetic Streptavidin beads}

Staged embryos expressing GFPslam (as well as endogenous Slam) were collected as mentioned in the previous section (2.2.2.5). 40 $\mu$ l of Streptavidin MyOne T1 dynabeads (Streptavidin paramagnetic beads) were washed twice with 1 X PBS. About 5000 embryos were lysed in $500 \mu$ l of RIPA buffer and input sample taken. The lysate was then incubated with $\sim 45 \mathrm{nM}$ final concentration of biotinylated GFP-binding protein for one hour on ice (biotinylated-GBP - a gift from Prof. Dirk Görlich). The lysate with biotinylated-GBP was added to the beads and rotated on a wheel at $4^{\circ} \mathrm{C}$ for $1 \mathrm{~h}$. The beads were settled down and unbound sample taken. The beads were washed thrice with RIPA buffer. The bound protein was eluted by adding $1 \mathrm{X}$ Lämmlli buffer and heating at $95^{\circ} \mathrm{C}$ for $5 \mathrm{~min}$.

\subsubsection{Fly embryo methods}

\subsubsection{Fractionation of embryos}

\section{a) WT embryos followed by western and qPCR:}

Approximately 7500 staged embyros were lysed in $1.5 \mathrm{ml}$ of fractionation buffer (Refer to 2.1.2 under 'For fractionation' for buffer recipes). The lysate was centrifuged twice at $2500 \mathrm{rpm}$ for $5 \mathrm{~min}$ at $4^{\circ} \mathrm{C}$ to pellet the nuclei. $1.3 \mathrm{ml}$ supernatant (total lysate) was transferred into a new eppendorf. 'Total lysate' protein sample was taken such that the final concentration in 1X Lämmlli buffer was 1embryo/ $\mu \mathrm{l}$. For 'Total lysate' RNA sample, $200 \mu \mathrm{l}$ ( 1000 embryos) was used and immediately processed for RNA isolation (For protocol, refer to 2.2 .3 .2$)$. $1 \mathrm{ml}$ of the remaining total lysate ( 5000 embryos) was transferred into a new eppendorf tube and centrifuged at $14,000 \mathrm{rpm}$ for $15 \mathrm{~min}$ at $4^{\circ} \mathrm{C}$. The lipid layer was generously removed by an aspirator. $500 \mu$ of supernatant (Cytoplasm) was carefully transferred into a new eppendorf. The rest of the supernatant was discarded and the pellet (Membrane) was re-suspended in $1 \mathrm{ml}$ of fresh fractionation buffer until a fine suspension was formed. The 'Cytoplasm' and the 'Membrane' tubes were centrifuged again at $14,000 \mathrm{rpm}$ for $15 \mathrm{~min}$ at $4^{\circ} \mathrm{C}$. If there was a significant pellet in the 'Cytoplasm' fraction, it was included in the 'Membrane' tube. The wash step was repeated again. After the second wash, 'Cytoplasm' protein and RNA sample was taken from the supernatant in a similar manner to the 'Total lysate' sample. The 'Membrane' pellet was re-suspended in $500 \mu \mathrm{l}$ fractionation buffer. 
'Membrane' protein sample was taken such that the final concentration in 1X Lämmlli was 3 embryos/ $\mu \mathrm{l}$. For the 'Membrane' RNA sample, $100 \mu \mathrm{l}$ (membrane derived from $\sim 1000$ embryos) was used. All protein samples were heated immediately at $95^{\circ} \mathrm{C}$ for 5 min and kept at room temperature until the end of protocol. They were loaded on an $8 \%$ SDS gel and a western blot for Slam and $\alpha$-tubulin was carried out. The detection of Slam was done by IRDye 800 secondary antibody while the detection of $\alpha$-tubulin was done by IRDye 680 secondary antibody on the same membrane.

Fractionation of genomic GFPslam embryos was carried out exactly in the same manner, except that the RNA samples were not taken.

\section{b) Fractionation followed by high salt membrane extraction:}

Fractionation of wild-type embryos was carried out in a similar manner as described above. Only protein samples were taken. After taking the 'Membrane' protein sample, $\mathrm{NaCl}$ was added to a final concentration of $1 \mathrm{M}$. The sample was kept on ice with intermittent resuspension for $5 \mathrm{~min}$. It was then centrifuged at 14,000 rpm for $15 \mathrm{~min}$ at $4^{\circ} \mathrm{C}$. The supernatant sample was dissolved in $1 \mathrm{X}$ Lämmlli such that $20 \mu \mathrm{l}$ sample contained 250 embryos.

\subsubsection{RNA isolation following fractionation of embryos}

The 'Membrane' RNA lysate sample (100 $\mu$ l volume) was made up to $200 \mu \mathrm{l}$ using fractionation buffer to make the volume equal to the 'Total lysate' and 'Cytoplasm' fractions. $20 \mu \mathrm{l}$ of $10 \%$ SDS and $1 \mu \mathrm{l}(20 \mu \mathrm{g} / \mu \mathrm{l})$ glycogen and $200 \mu \mathrm{l}$ Phenol:Chloroform:Isoamyl alcohol was added to the lysates, mixed well and centrifuged at $14,000 \mathrm{rpm}$ for $5 \mathrm{~min}$ at $4^{\circ} \mathrm{C}$. The upper phase was transferred into fresh tubes and $200 \mu \mathrm{l}$ chloroform was added and mixed well. The samples were centrifuged at $14,000 \mathrm{rpm}$ for $5 \mathrm{~min}$ at $4^{\circ} \mathrm{C}$. The upper phase was transferred to fresh tubes and $40 \mu \mathrm{l}$ of $3 \mathrm{M} \mathrm{NaAc}$ (DEPC-treated) was added and mixed. Then $500 \mu \mathrm{l}$ of $100 \%$ Ethanol was added, mixed and kept at $-20^{\circ} \mathrm{C}$ overnight. The RNA was pelleted by centrifuging at $14,000 \mathrm{rpm}$ for $30 \mathrm{~min}$ at $4^{\circ} \mathrm{C}$, washed with $100 \mu \mathrm{l} 80 \%$ DEPC-treated Ethanol and centrifuged again at $14,000 \mathrm{rpm}$ for $15 \mathrm{~min}$ at $4^{\circ} \mathrm{C}$. The pellet was dried under a hood for 10-15 min and dissolved in $40 \mu \mathrm{l}$ RNAse-free water. The samples were stored at $20^{\circ} \mathrm{C}$. 


\subsubsection{Embryo fixation}

Embryos were dechorionated as mentioned earlier (2.2.2.3a) and collected into a net and transferred into a scintillation vial with $5 \mathrm{ml}$ heptane and $4.5 \mathrm{ml}$ PBS. To this, 0.5 $\mathrm{ml}$ of $37 \%$ formaldehyde was added (final concentration of $4 \%$ ). The embryos were fixed for $20 \mathrm{~min}$ with constant shaking. The lower phase was removed and the embryos were washed twice by adding and removing $4.5 \mathrm{ml}$ PBS. $5 \mathrm{ml}$ of methanol was added to the embryos and the vial was vortexed for $30 \mathrm{sec}$ to devitellinise or 'pop' the embryos. The popped embryos sink to the bottom. They were collected using a Pasteur pipette and transferred to a fresh eppendorf tube. They were washed twice with methanol and also stored in methanol at $-20^{\circ} \mathrm{C}$.

\subsubsection{Immunostaining of embryos}

The embryos stored in methanol were rinsed thrice and washed twice for 5 min in PBT. Blocking was carried out for 1 hour in $1 \mathrm{ml}$ of $5 \%$ BSA in PBT. The primary antibodies were added in the respective dilutions in PBT and embryos were incubated at $4^{\circ} \mathrm{C}$ with constant rotation. Embryos were then rinsed thrice in PBT and given four 15 min washes. Appropriate secondary antibodies were added in PBT and incubated for 2 hours. Embryos were again rinsed thrice in PBT and given four 15 min washes. Embryos were stained with DAPI for $5 \mathrm{~min}$, rinsed thrice with PBT, washed once for 5 min with PBT and were mounted using aquapolymount mounting medium.

\subsubsection{Fluorescence in-situ hybridisation (FISH)}

a) Preparation of Dig-labelled slam mRNA probe:

Following components were mixed together and incubated at $37^{\circ} \mathrm{C}$ for $2 \mathrm{~h}$ :

$\begin{array}{lll}\text { Template DNA }(1 \mu \mathrm{g} / \mathrm{ml}) & - & 1 \mu \mathrm{l} \text { (linearised OTslam plasmid) } \\ \text { 10X NTP+Dig labelling mix } & - & 2 \mu \mathrm{l} \\ \text { 10X transcription buffer } & - & 2 \mu \mathrm{l} \\ \text { T7 RNA polymerase }(\sim 200 \mathrm{U} / \mu \mathrm{l}) & - & 1 \mu \mathrm{l} \\ \text { RNAse inhibitor } & - & 0.5 \mu \mathrm{l}\end{array}$


DEPC-treated water

$13.5 \mu \mathrm{l}$

The RNA was treated with $2 \mu \mathrm{l}$ DNasel $(10 \mathrm{U} / \mu \mathrm{l})$ and incubated for $15 \mathrm{~min}$ at $37^{\circ} \mathrm{C}$. The RNA was then purified using phenol-chloroform extraction (Refer to 2.2.1.5). The pellet was dissolved in $20 \mu \mathrm{l}$ DEPC-treated water and stored at $-20^{\circ} \mathrm{C}$.

\section{b) in-situ hybridisation of Drosophila embryos:}

Day 1: Dechorionated and fixed embryos were rinsed thrice with PBT and washed twice for $5 \mathrm{~min}$ each. The embryos were incubated for $10 \mathrm{~min}$ in 1:1 hybridisation solution:PBT. Then the embryos were incubated in hybridisation solution (hyb-sol) for $10 \mathrm{~min}$. The embryos were prehybridised in hyb-sol for 1 hour at $57^{\circ} \mathrm{C}$. $1 \mu \mathrm{l}$ of slam probe and $1 \mu \mathrm{l}$ of tRNA was mixed in $20 \mu \mathrm{DEPC}-\mathrm{H}_{2} \mathrm{O}$ and heated at $95^{\circ} \mathrm{C}$ for $4 \mathrm{~min}$. The probe was chilled rapidly in ice-water. $200 \mu$ of chilled hybridisation solution was added to this. After the prehybridisation of embryos, the probe solution was added to the embryos and incubated overnight at $57^{\circ} \mathrm{C}$.

Day 2: The probe solution was removed and the embryos were rinsed thrice with prewarmed hyb-sol. The embryos were then give three $30 \mathrm{~min}$ washes in hyb-sol at $57^{\circ} \mathrm{C}$. The embryos were then given four 10 min washes with $4: 1$ hyb-sol/PBT (at $57^{\circ} \mathrm{C}$ ), $3: 2$ hyb-sol/PBT (at $57^{\circ} \mathrm{C}$ ), 2:3 hyb-sol/PBT(at $57^{\circ} \mathrm{C}$ ) and $1: 4$ hyb-sol/PBT (at room temperature) respectively. Then the embryos were washed twice, 20 min each with 1\% BSA in PBT at room temperature. Digoxigenin antibody coupled with peroxidase was added to the embryos in a 1:200 dilution in PBT and incubated for 2 hours at room temperature. The embryos were rinsed thrice with PBT and washed four times, 15 min each. TSA-Cy3 stock solution was diluted 1:200 in reaction buffer and incubated with embryos for $\sim 1 \mathrm{~min}$, protected from light. The staining reaction was stopped by washing with PBT. Then primary antibodies for protein staining were added in appropriate dilutions in PBT and incubated overnight, protected from light.

Day 3: Immunostaining was carried out for proteins according to the protocol mentioned in 2.2.3.4. Immunostaining was carried out in dark. 


\subsubsection{Generation of germline clones}

Germline clones in the Drosophila germline were created mainly according to Chou and Perrimon, 1992. Heat shock for the expression of Flippase was carried out at $37^{\circ} \mathrm{C}$ for 60 min on two consecutive days after 24 hours post-hatching.

\subsubsection{Fly genetics}

slam germline clones were generated using $\mathrm{Df}(2 \mathrm{~L})$ slam $\mathrm{Frt}(2 \mathrm{~L})$ slam 5'rescue chromosome.

nullo,slam double mutants were created by crossing females of $\mathrm{Df}(1)$ nullo6F/hsFlp[122]; Df(2L)slam Frt[2L] slam5'rescue/ovo[2L] with Df(2L)BSC5/CyO, hb-lacZ males.

To test whether GFPslam68 can rescue viability, Df(2L)slam slam5'rescue/CyO; GFPslam/TM3 females were crossed with Df(2L)slam slam3'rescue/CyO; +/+ males. Rescued flies would consist of straight wings.

To test whether GFPslam68 can rescue cellularisation phenotype, Df(2L)slam Frt[2L] slam5'rescue/ovo[2L]; GFPslam68 females were crossed with Df(2L)BSC5/CyO, hblacZ males. All resultant embryos consisted of a single copy of GFPslam68 as maternal contribution. Half of the embryos (lacZ positive) had a zygotic rescue from a single copy of endogenous slam. One-fourth of the embryos (lacZ negative) contained a single copy of zygotic GFPslam68 while the other one-fourth (lacZ negative) contained no zygotic slam/GFPslam68.

\subsubsection{Induction of shibire phenotype}

shibire phenotype was induced in embryos derived from heterozygous shi(ts) females by keeping the embryos after dechorionation at $32^{\circ} \mathrm{C}$ in a water bath for 30 minutes. The embryos were then fixed. For live-imaging, embryos were dechorionated and glued on to a glass coverslip and covered with halocarbon oil. Then the coverslip was kept on a metal block heated to $32^{\circ} \mathrm{C}$ for $10 \mathrm{~min}$ and the imaging was carried out at $32^{\circ} \mathrm{C}$ at the LSM microscope. 


\subsubsection{Microinjection of protein/drug into embryos}

Embryos were dechorionated, dried in a desiccation chamber for $\sim 8 \mathrm{~min}$, covered with halocarbon oil and injected posteriorly with 50-100 pl of protein/drug. Imaging of GFPslam was carried out after 10 min of injection.

\subsubsection{Microscopy}

\subsubsection{Live-imaging of fly embryos}

Embryos were dechorionated, glued on to a coverslip and covered with halocarbon oil. Fluorescent live-images of GFPslam in buffer-injected and cycloheximide-injected embryos were taken at the LSM with a $63 \mathrm{X}$ oil objective. All other fluorescent liveimages were taken at the spinning disc with a $40 \mathrm{X}$ oil objective. Differential interference contrast microscopy was carried out at the spinning disc microscope with a $25 \mathrm{X}$ oil objective.

\subsubsection{Fluorescence recovery after photobleaching (FRAP)}

Due to the changing Z-position of the furrow during cellularisation, a range of Z-stacks were recorded. Bleaching of GFPslam was carried out in a given area using $100 \%$ laser power and 50 iterations at a scan speed of 5 . The appropriate Z-stacks were merged for analysis. For the experiment with FRAP of GFPslam at different time-points of cellularisation, fluorescence of a small membrane area outside and inside of the bleached region was measured. For FRAP of GFPslam in shibire background, fluorescence intensity of only 'old borders' were taken into account. For the experiment with nuf mutants, due to increased membrane dynamics, fluorescence was measured at the complete furrow canal area outside and inside of the bleached region. The background intensity was subtracted from the total fluorescence. For all experiments, the starting point of the fluorescence was normalised to 1. Linear regression of fluorescence recovery was used to calculate the rate of fluorescence recovery.

\subsubsection{Laser ablation of centrosomes}

Embryos expressing SAS6GFP and GFPslam were aligned on agar, glued on to a coverslide and covered with halocarbon oil. Very little glue was used since it makes 
the UV laser less efficient. Ablation of centrosomes was carried out with a $355 \mathrm{~nm}$ pulsed UV laser (70 $\mu \mathrm{J} /$ pulse, $4 \times 200 \mathrm{~ms}, 30 \%$ ) in the anaphase of cell cycle 13 , before the centrosome duplication. Time-lapse axial image stacks were recorded following ablation and subsequently merged with Fiji software. 


\section{RESULTS}

\subsection{Factors contributing to the accumulation of Slam protein at the furrow canal}

\subsubsection{Centrosomes define the site of invagination}

Centrosomes are microtubule-organizing centres and thus have been implicated in the specification of cleavage furrow. It has been shown that centrosomes alone, in the absence of nuclei, can induce formation of pole cells in the early Drosophila embryo (Raff and Glover, 1989). Hence, we asked whether centrosomes are the source of the initial signal for Slam accumulation and the formation of furrow during cellularisation.

It has been described that overexpression of SAS6 induces de novo formation of multiple centriole-like structures in syncytial embryos (Peel et al., 2007). These structures are formed due to additional replications of centrioles in the same cell cycle. These ectopic centriole precursors are able to reorganise microtubules (RodriguesMartins et al., 2007). Previously in our lab, it was shown that these ectopic centriole precursors are sufficient for the specification of a new furrow at the onset of cellularisation (Fig 3.1 A and B, Dr. Philip Laupsien, PhD Dissertation). Slam was able to localise to the furrow canal, though it was distributed all over the basal membrane, probably due to premature basal closure of the furrows. The ectopic furrows also displayed localisation of the lateral membrane marker Dlg, showing that the invaginating membrane is polarised. Other furrow canal markers (PDZ domain of RhoGEF2, Actin) were also found to be localised indicating that the furrows were functional (Acharya et al., 2014; Dr. Christian Wenzl, PhD dissertation).

The ectopic furrows undergo a premature basal closure, perhaps but not exclusively due to the absence of nuclei. When we observed at a very early stage of cellularisation, we could find Slam localised in a proper furrow morphology (Fig. 3.1 C). This suggests that centrosomes are able to specify the site of furrow invagination, and is able to restrict Slam in the initial stage of cellularisation.

We wondered whether some of these ectopic centriole precursors were formed as a result of nuclear fallout in earlier cell cycles. This would mean that the furrow- 

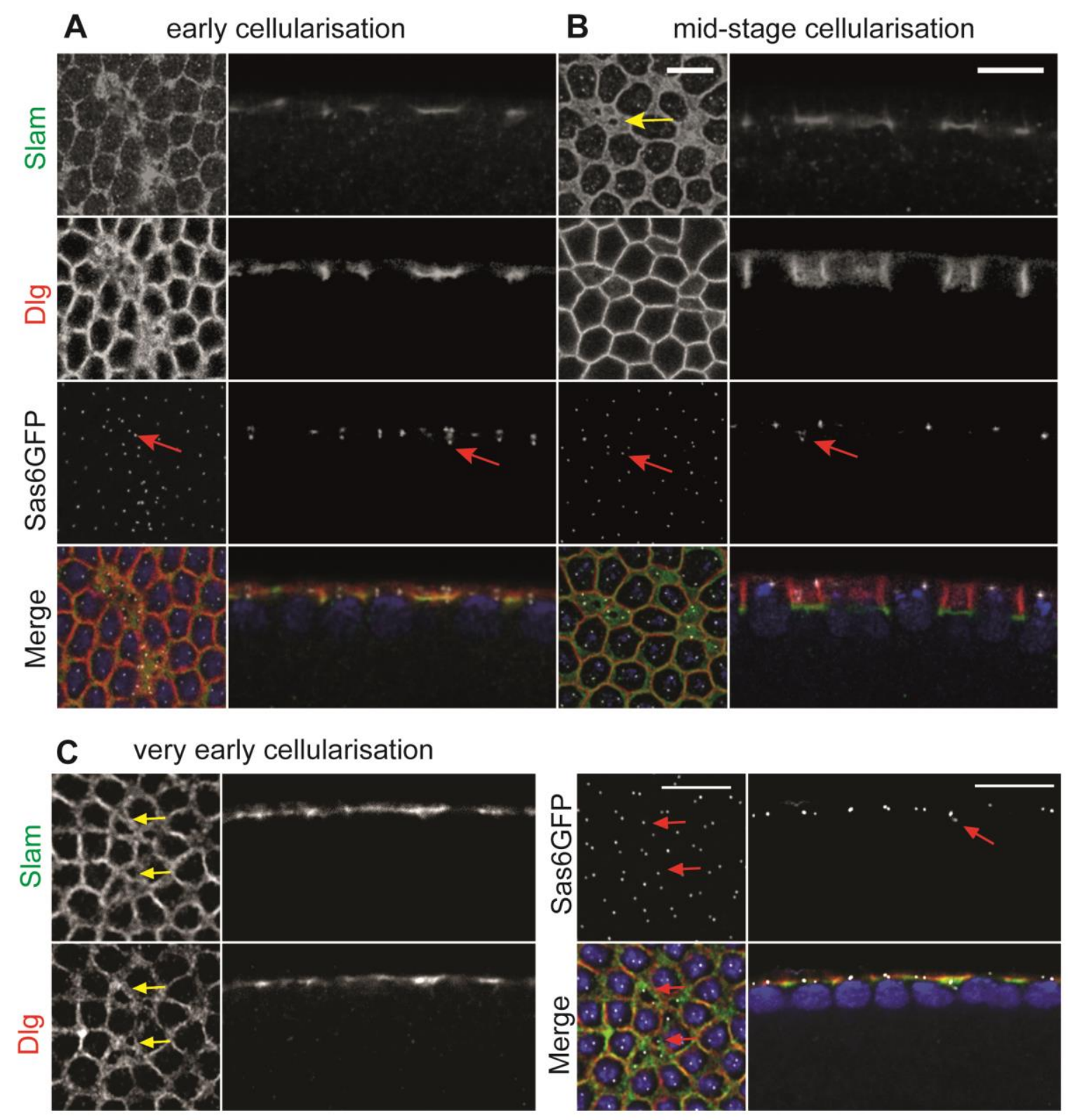

Fig 3.1 Lonesome centrosomes are capable of specifying ectopic furrows. Embryos overexpressing SAS6GFP display lonesome centrosomes that lack nuclei (red arrows). These lonesome centrosomes are able to specify ectopic furrows around them (yellow arrows), as marked by accumulation of Slam (green) and Dlg (red). (A and B, Dr. Philip Laupsien, PhD Dissertation) Scale bar $10 \mu \mathrm{m}$.

specification is carried out by the nucleus rather than the centrosome. To rule out this possibility, live imaging of syncytial embryos expressing SAS6-GFP under a ubiquitin promoter and GFP-slam driven by a maternal tubulin promoter was carried out. It was 


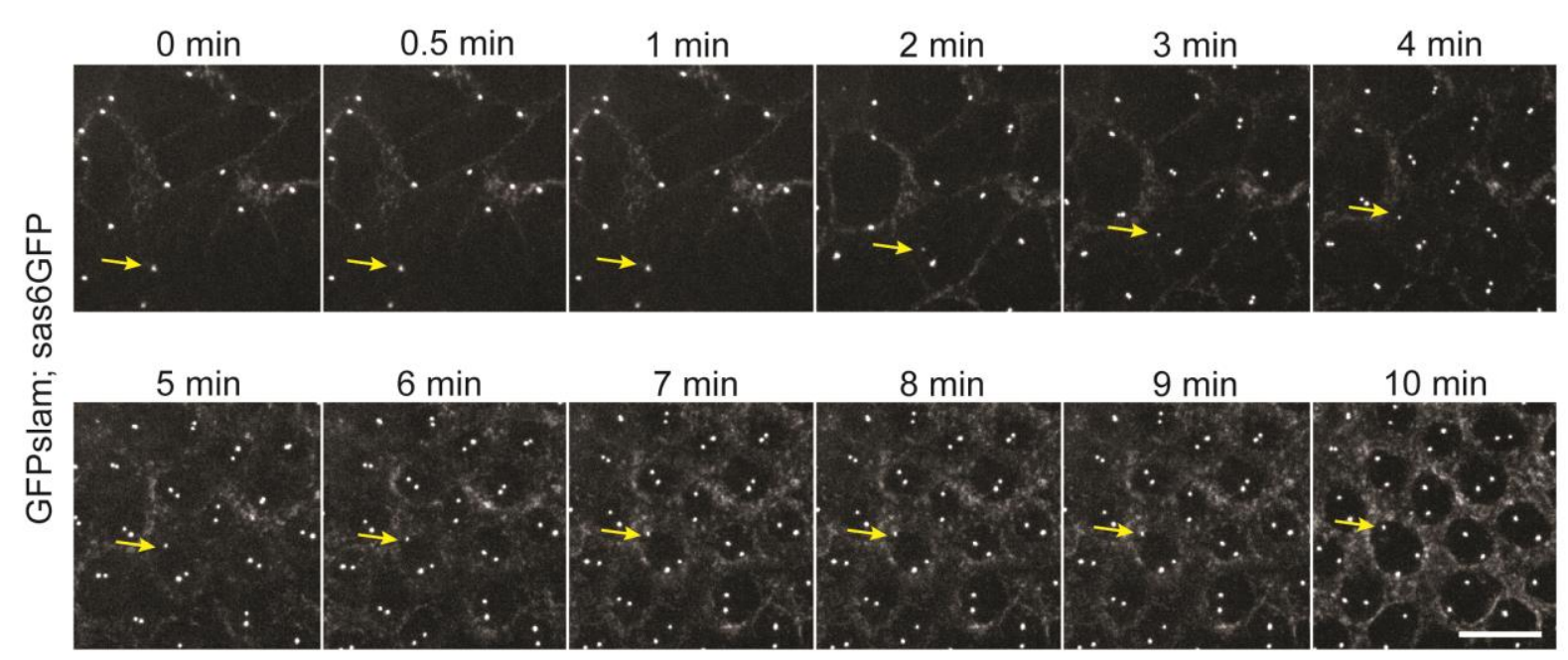

Fig 3.2 SAS6GFP overexpression results in extra centrosomes in the embryo due to additional centrosomal divisions. Embryos expressing SAS6GFP and GFPslam were followed from anaphase of cycle 13 until the onset of cellularisation. Extra centrosomes result from additional replication of centrosomes (yellow arrow). Scale bar $10 \mu \mathrm{m}$.

found that the ectopic centriole precursors capable of specifying a new furrow, were formed de novo by extra divisions of the centrosomes and were not a consequence of nuclear fallout (Fig. 3.2). When observed live, we found that both duplicate lonesome centrosomes (Fig 3.3 A) and singular lonesome centrosomes (Fig 3.4 B) were capable of specifying a new furrow.

Furthermore, we asked whether furrow specification was lost in the absence of a centrosome. To answer this question, we tried to ablate a centrosome and see the effect on GFP-slam accumulation. The ablation was carried out in the anaphase of cycle 13 before the formation of the new furrow. However, only partial ablation could be achieved due to the susceptibility of the adjacent membrane to a higher intensity/duration of laser. Despite this limitation, we could observe a loss in the centrosome's ability to specify a new furrow (Fig 3.4). This suggests that we were able to functionally ablate the centrosome to a large extent. Although the ablation was carried out before the formation of the nuclear membrane, we could not rule out the possibility that a nuclear fallout could have been induced during ablation. However, since we showed earlier that centrosome is able to specify the site of the furrow even in the absence of a nucleus, a nuclear fallout is inconsequential to furrow-specification. 


\section{A. Duplicate lonesome centrosomes}

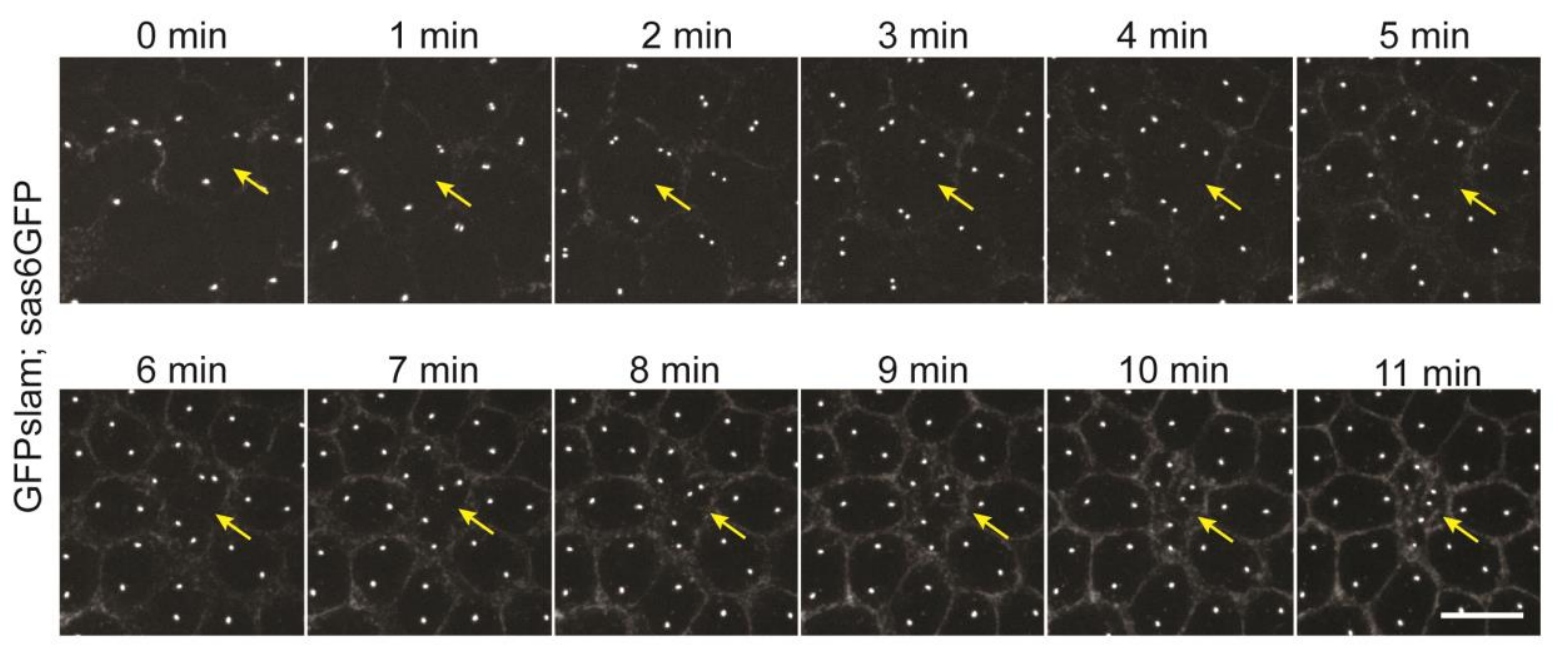

B. Singular lonesome centrosomes

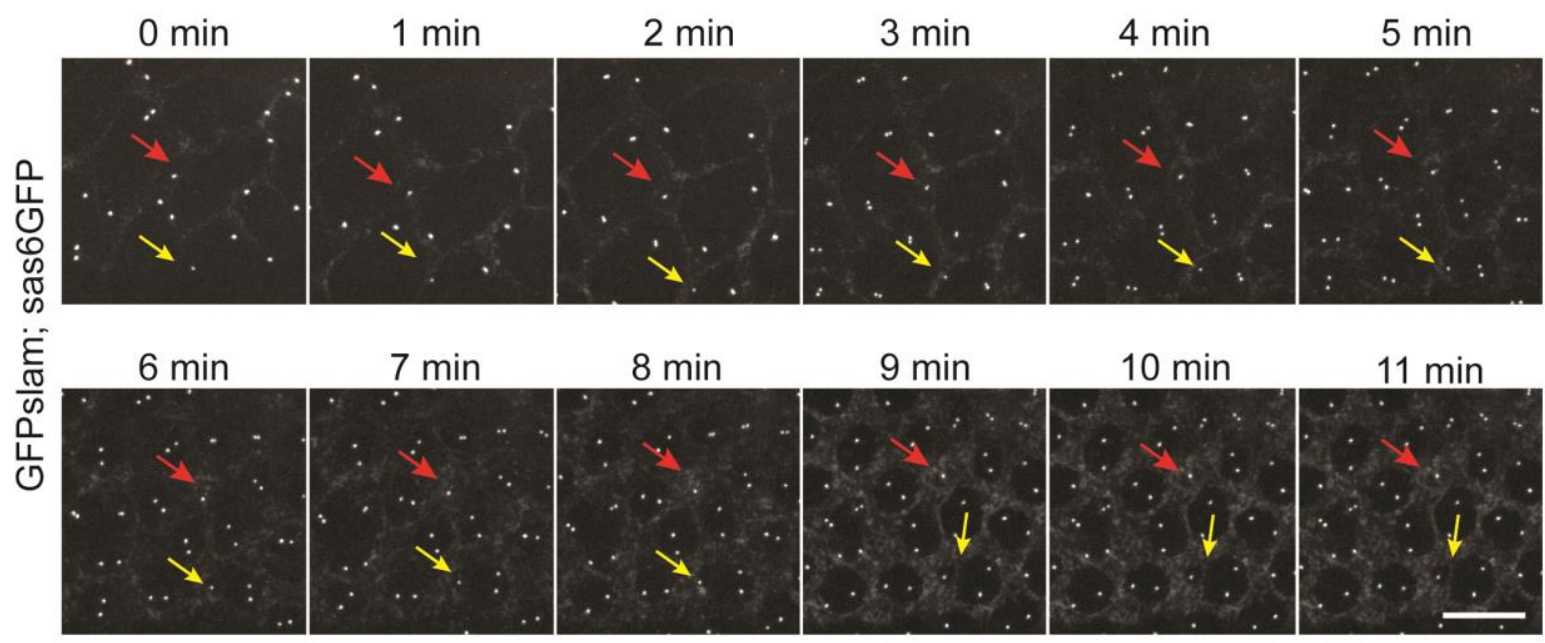

Fig 3.3 GFPslam accumulates around the additional duplicate/singular lonesome centrosomes to form ectopic furrows in SAS6GFP embryos. Duplicate lonesome centrosomes (A, yellow arrows) and Singular lonesome centrosomes (B) both are able to specify ectopic furrows. The red arrows in $B$ indicate a singular lonesome centrosome formed in an earlier cycle as indicated by GFPslam accumulation in cycle 13. The yellow arrows in B indicate a singular lonesome centrosome formed in cycle 13 as indicated by GFPslam accumulation in cycle 14 . Scale bar $10 \mu \mathrm{m}$. 


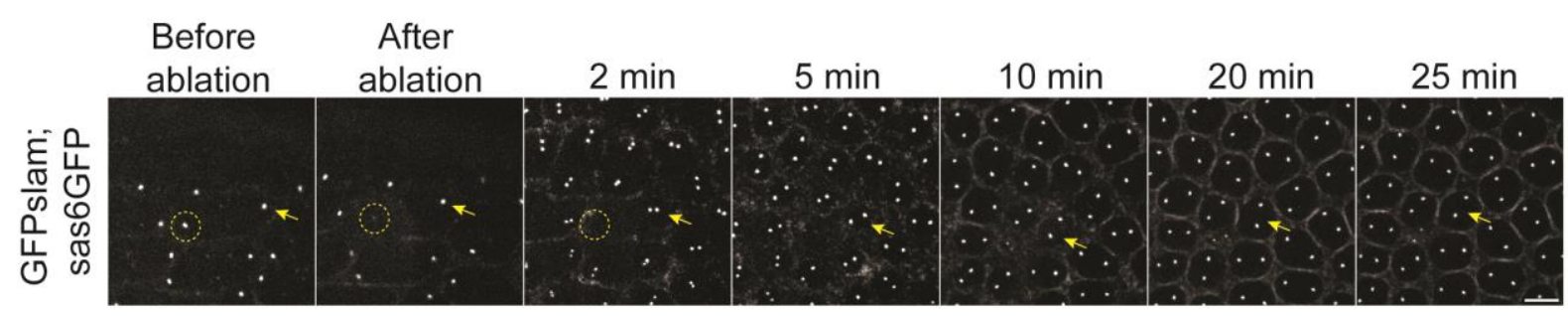

Fig 3.4 Functional ablation of a centrosome induces loss of its furrow-specifying ability. Centrosome was ablated in late mitosis using a pulsed UV-laser. The dashed-circle indicates the ablated centrosome. The yellow arrow indicates the centrosome/centrosome pair of the daughter nucleus. Scale bar $5 \mu \mathrm{m}$.

\subsubsection{Accumulation of Slam is dependent on vesicular transport}

Next, we wanted to find out the mechanism by which Slam protein is transported to the furrow canal. We considered two non-exclusive possibilities a) microtubuledependent transport b) vesicle-based transport. When GFPslam punctae were followed using live imaging, they showed a random movement instead of a characteristic directed movement seen in case of microtubule-dependent transport (Dr. Philip Laupsien, $\mathrm{PhD}$ dissertation). It has been shown that vesicle trafficking through the recycling endosome is necessary for cellularisation (Pelissier et al., 2003). This gave us an indication towards a direct or indirect involvement of vesicular transport in localising Slam.

One way of interfering with vesicular transport is to disrupt shibire (shi), the Drosophila homologue of Dynamin. We used a temperature-sensitive conditional mutant of shi which allowed us to induce the shi phenotype in a temporal manner (Pelissier et al., 2003). shi embryos were shifted to restrictive temperature $\left(32^{\circ} \mathrm{C}\right)$, about fifteen minutes earlier to the onset of cellularisation, before formation of the new furrow. GFPslam was followed live after the induction of shiphenotype. It was observed that GFPslam localisation to the site of the 'new furrow' was perturbed. Either it completely failed to localise or sometimes it showed partial localisation (Fig 3.5). In contrast, GFPslam accumulation in wild-type background was normal at $32^{\circ} \mathrm{C}$.

Interfering with shi function, however, also disrupts apical endocytosis of the plasma membrane and therefore membrane delivery to the new furrow during the slow phase of cellularisation (Lecuit and Wieschaus, 2000). The failure of accumulation of 


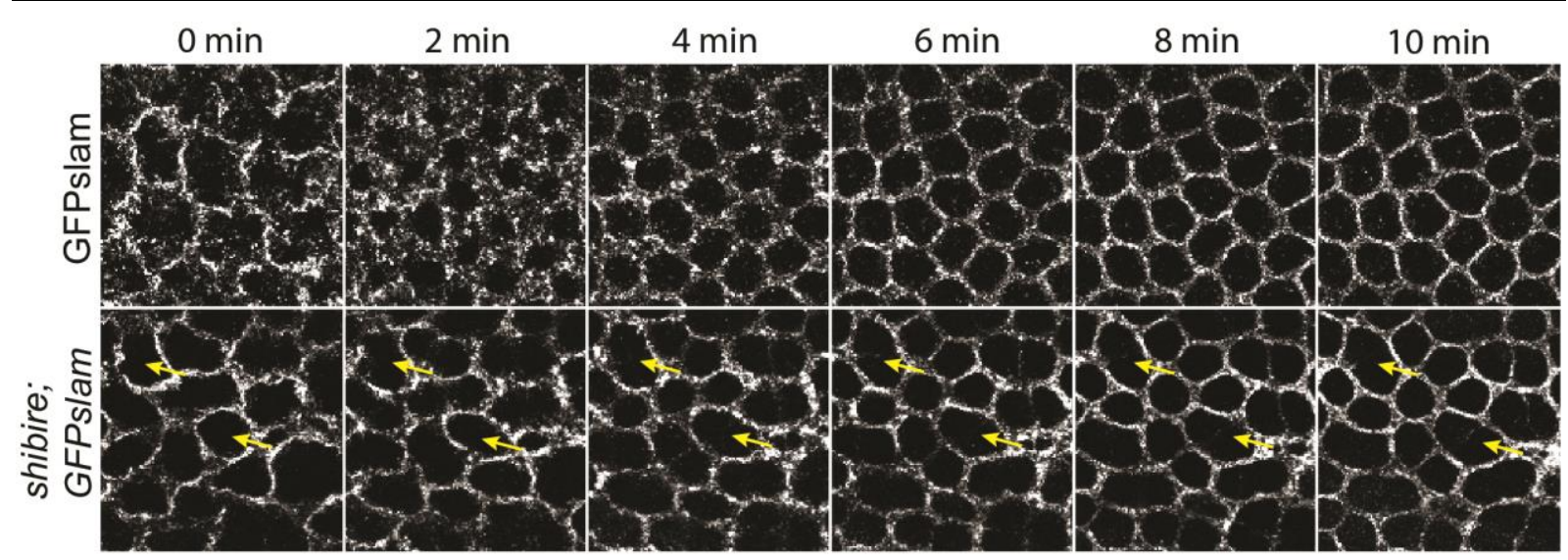

Fig 3.5 Slam accumulation at the furrow canal is dependent on vesicular transport. GFPslam accumulation at the 'new furrow' was followed using time-lapse recordings in embryos from shibire and wild type females expressing GFPslam at $32^{\circ} \mathrm{C}$. Yellow arrows indicate incompletely or absent "new" furrows.

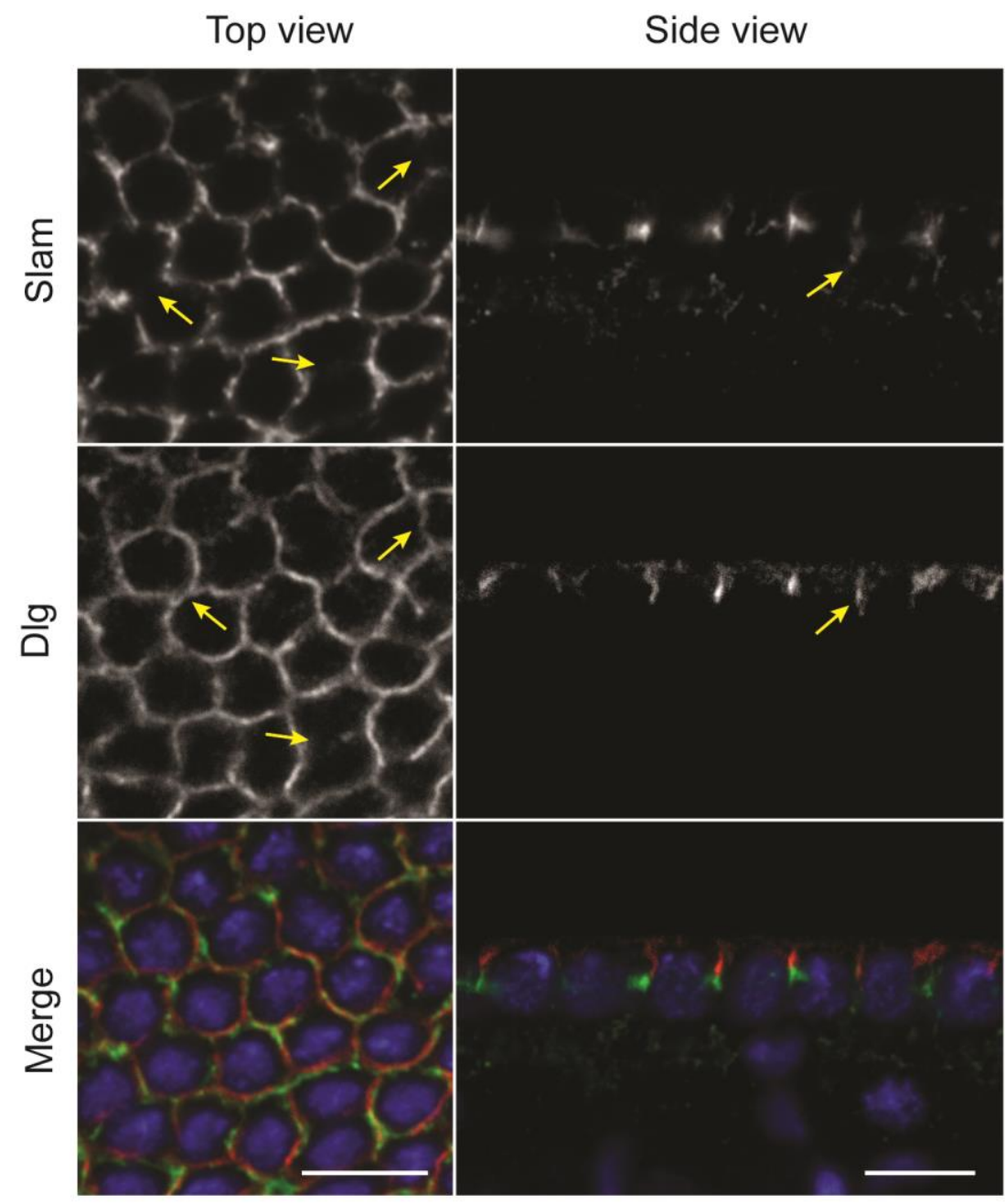

Fig 3.6 Loss of Slam from the furrow canal is a specific effect. Fixed and stained embryos from shibire females. The shibire phenotype was induced by keeping the embryos at $32^{\circ} \mathrm{C}$ for $30 \mathrm{~min}$ before fixation. The yellow arrows point to furrows where Slam is lost and Dlg is intact. Scale bar $10 \mu \mathrm{m}$. 
Slam at the furrow canal therefore could also be due to absence of a membrane structure. To check this, we shifted the shi conditional mutants to the restrictive temperature, fixed and stained them for Slam and Dlg (a lateral membrane marker). Dlg is able to localise to the furrow in the absence of Slam (Acharya et al., 2014; Dr. Philip Laupsien, PhD dissertion). It was found that Slam was lost from the 'new border' before the loss of Dlg (Fig 3.6). Therefore it can be concluded that loss of Slam from the furrow canal was not a consequence of disintegration of the membrane structure.

To investigate the specific role of recycling endosome in Slam protein transport, we stained wild-type embryos during cellularisation for Slam and Rab11 to see whether we find a colocalisation of Slam protein with a subset of Rab11 vesicles (Fig 3.7 A). Rab11 was observed to be primarily localising at the pericentriolar region defining the recycling endosome, as described in Riggs et al., 2003. Slam was not found in this region. When we focussed at the furrow canal where Slam is known to localise, Rab11 did not show any specific localisation.

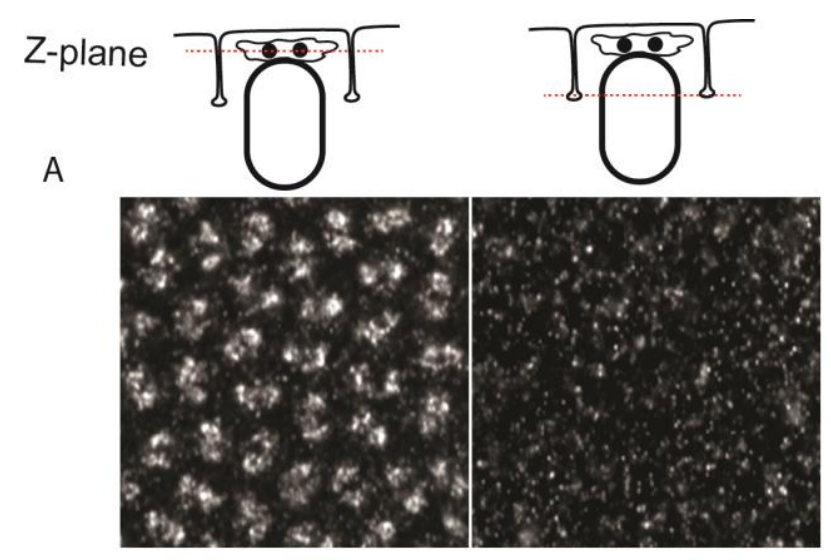

Rab11 immunostaining

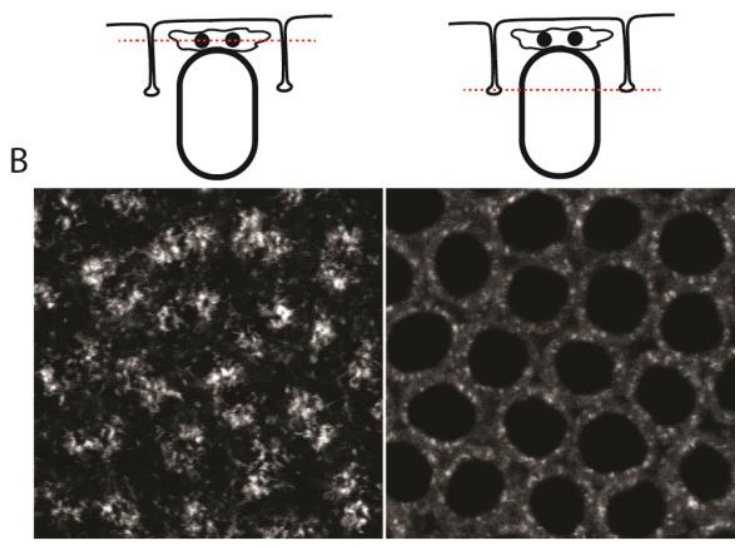

Rab11-YFP live-imaging

Fig 3.7 Rab11 is associated with the perinuclear recycling endosome and the recycling endosome vesicles. Rab11 shows a different localisation near the furrow canal in fixed (A) and live embryos (B). All images represent the top view of the embryo. The red, dashed line in the illustration indicates the plane of intersection of the Z-stack.

We observed embryos expressing Rab11YFP to see if additional Rab11 vesicles were detectable via live imaging (Fig 3.7 B). Rab11 showed similar accumulation at the recycling endosome as seen in the immunostaining pictures. However, additional Rab11 punctae were observed around the furrow canal, which were not visible via immunostaining. 
We followed GFPslam and Rab11YFP live to see whether there is any colocalisation at any stage with the additional Rab11 punctae around the furrow canal. No colocalisation was observed at any time point. Rab11YFP punctae were distributed in the cytoplasm whereas GFPslam was always found associated with the membrane (Fig 3.8). This suggests that Slam is not transported to the site of invagination on Rab11 vesicles.

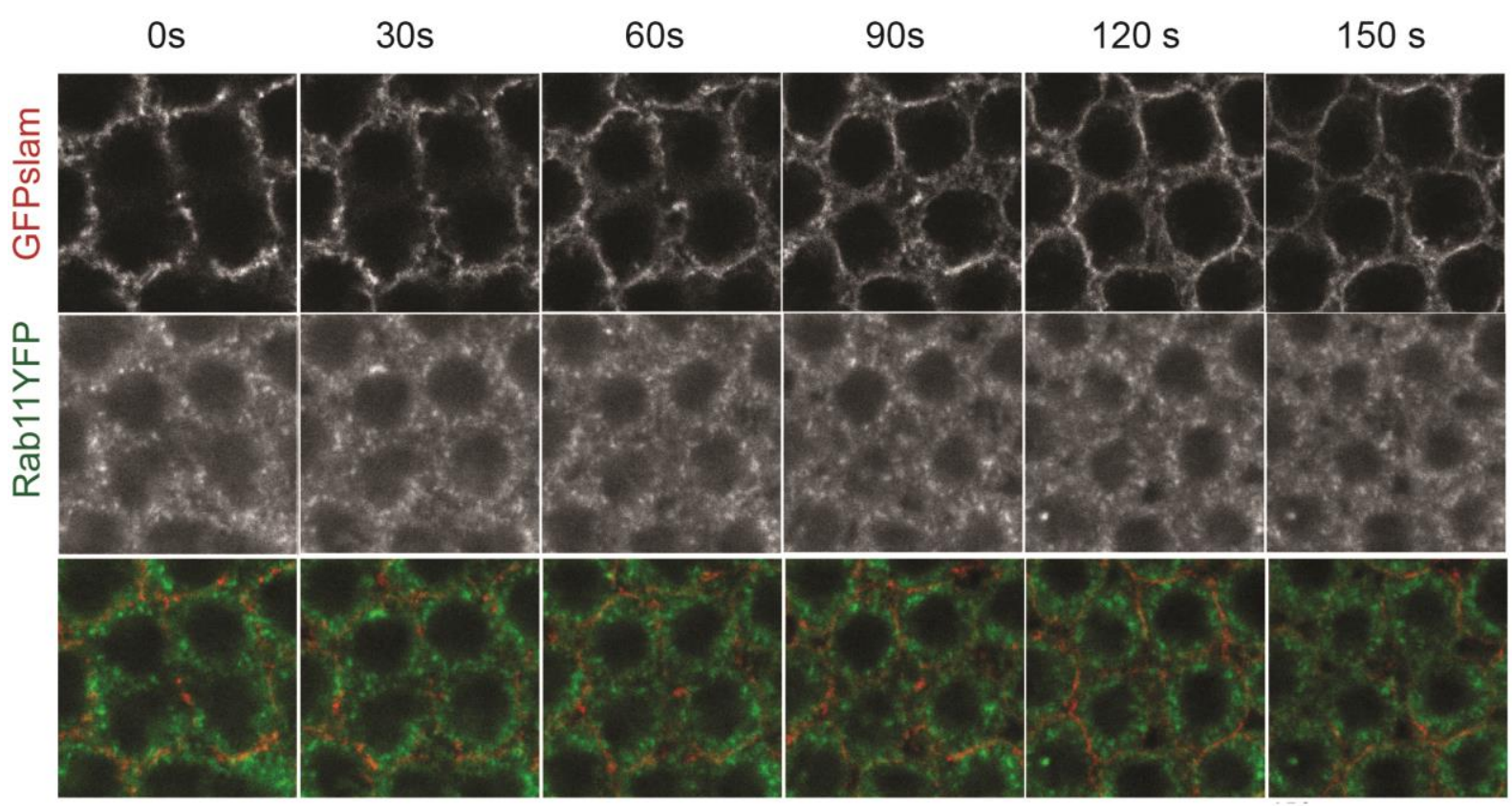

Fig 3.8 Slam is not transported on Rab11 vesicles. Embryos expressing GFPslam (red) and Rab11YFP (green) were followed from late cycle 13 to the onset of cellularsation using time-lapse recordings. (Imaging and image processing by Prof. Jörg Großhans).

Rab11 transheterozygote mutants however, show a reduced accumulation of Slam at the furrow canal (Dr. Philip Laupsien, PhD dissertation). In the absence of direct transport of Slam via the recycling endosome, we examined a functional link. It has been previously reported that in embryos derived from nuf homozygous females RhoGEF2 localisation at the furrow canal is disturbed (Cao et al., 2008). Previously in our lab it had been shown that Slam is sufficient and necessary for the localisation of RhoGEF2 to the furrow canal (Wenzl et al., 2010). Hence we wondered whether the mislocalisation of RhoGEF2 in embryos from nuf females (henceforth referred to as nuf embryos) is due to mislocalisation of Slam.

nuf embryos show a variable phenotype where the furrow canal morphology and invagination rate ranges from normal to completely disrupted. At least $50 \%$ of the embryos showed a strong or delayed phenotype $(n=14)$. Fig 3.9 and 3.10 represent 
the range of phenotypes observed. We stained nuf embryos with Slam and Rab11 and found that as reported earlier (Riggs et al., 2003), Rab11 localisation was dependent upon nuf. There was a visible reduction in Rab11 pericentrosomal accumulation in the mild cases and a complete absence of Rab11 at the region in the severe cases. In the severe cases, Slam was found strongly mislocalised to the apical and lateral regions.

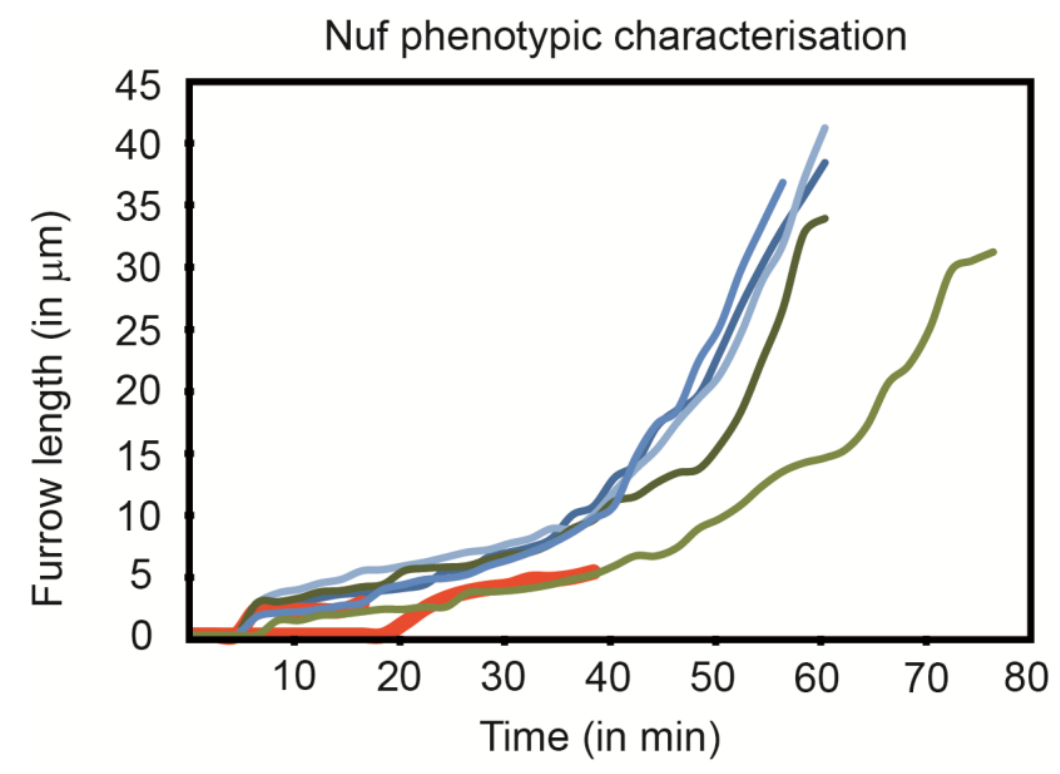

Fig 3.9 Graph depicting the variability of nuf embryos. The furrow length with respect to time was plotted for nuf embryos $(n=7)$. The blue lines represent mild/normal phenotypes, the green lines represent delayed phenotype and the orange lines represent severe phenotype. Scale bar $10 \mu \mathrm{m}$. 


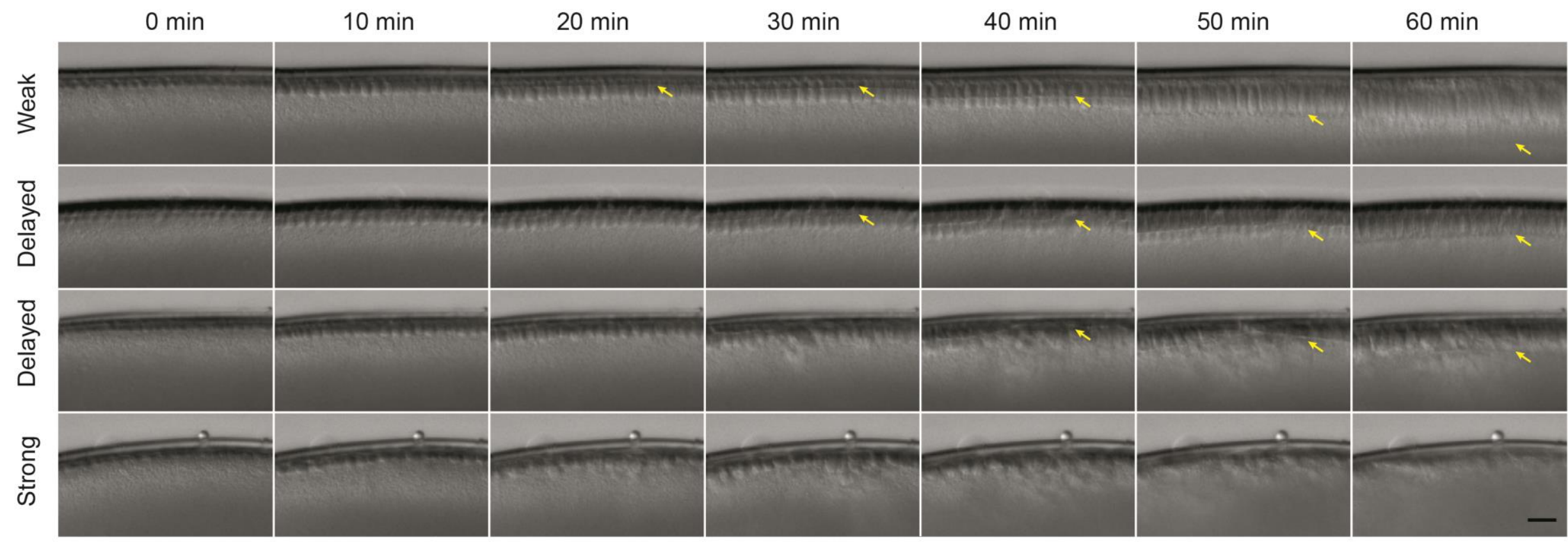

Fig 3.10 nuf embryos show variable phenotype ranging from mild to severe. Time-lapse movies of nuf embryos were obtained by Differential Interference Contrast (DIC) microscopy. Representive cases are depicted. Arrows point to the cellularisation front. Scale bar $10 \mu \mathrm{m}$. 
We further analysed the cases with delayed invagination and found that often Slam was mislocalised into the lateral membrane domain as shown by colocalisation with Dlg (Fig 3.11 A, yellow arrows). During cellularisation, slam, Rhogef2, nullo and dia control separation of lateral and basal domains (Sokac and Wieschaus, 2008b; Wenzl et al., 2010). So it is expected that in nuf embryos where Slam and therefore RhoGEF2 are mislocalised, the membrane polarisation would be disrupted. This was observed in the form of Dlg spreading into the basal domain (Fig 3.11 A, red arrows).

A

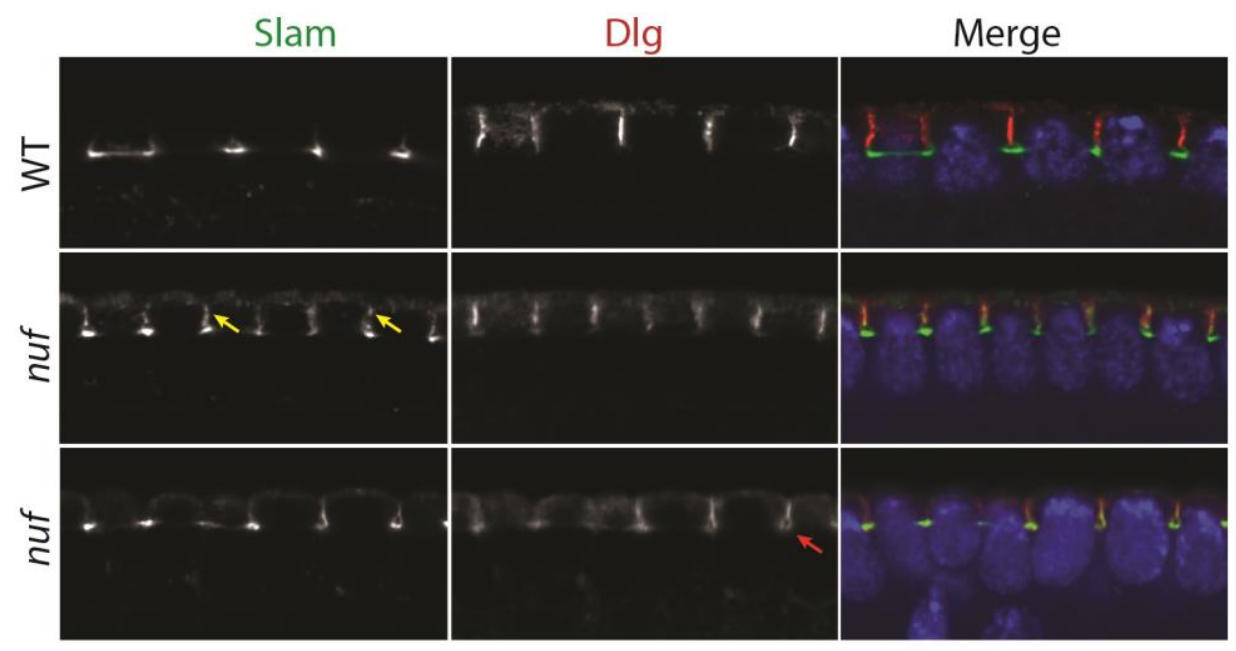

B

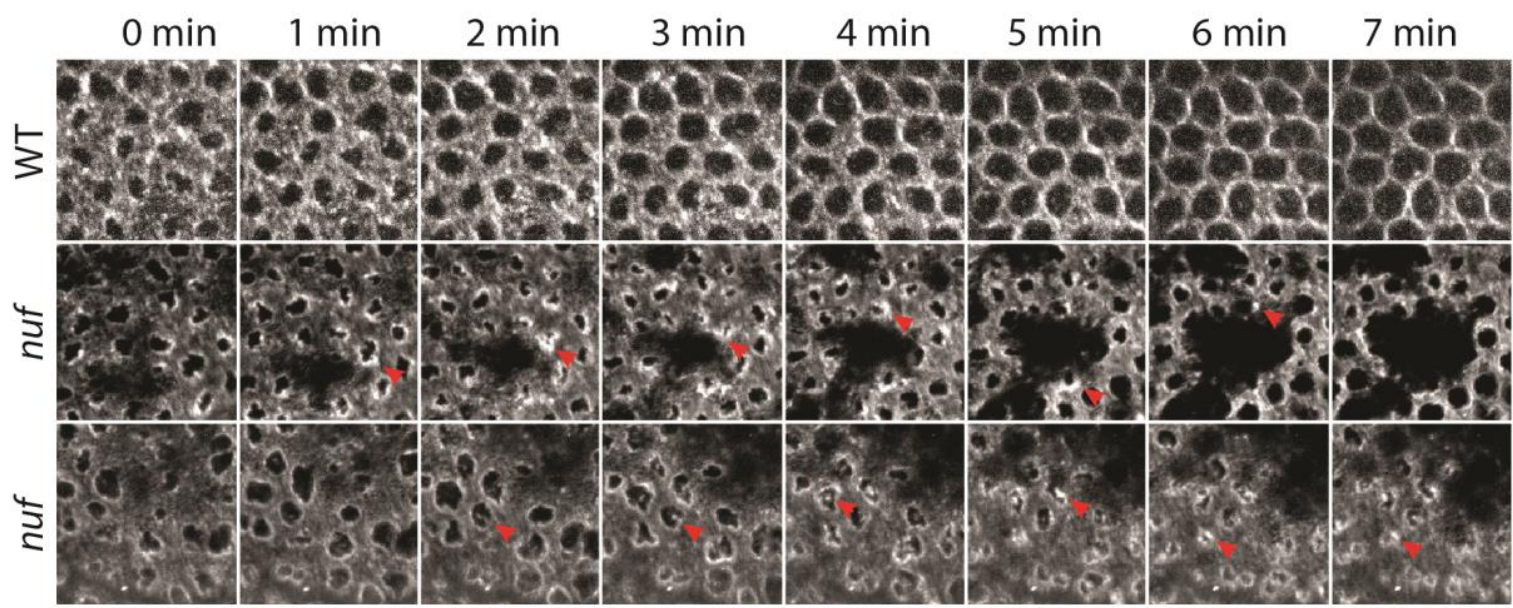

Fig 3.11 Slam is not restricted to the basal domain in nuf embryos. (A) Fixed nuf embryos were stained for Slam (green) and DIg (red). Slam is found mislocalised into the lateral domain (yellow arrows) and Dlg is occasionally spead into the basal domain (red arrows). (B) GFPslam was followed in WT and nuf embryos using time-lapse recordings. Red arrowheads point to the accumulations of GFPslam that persist much longer than in the WT. 

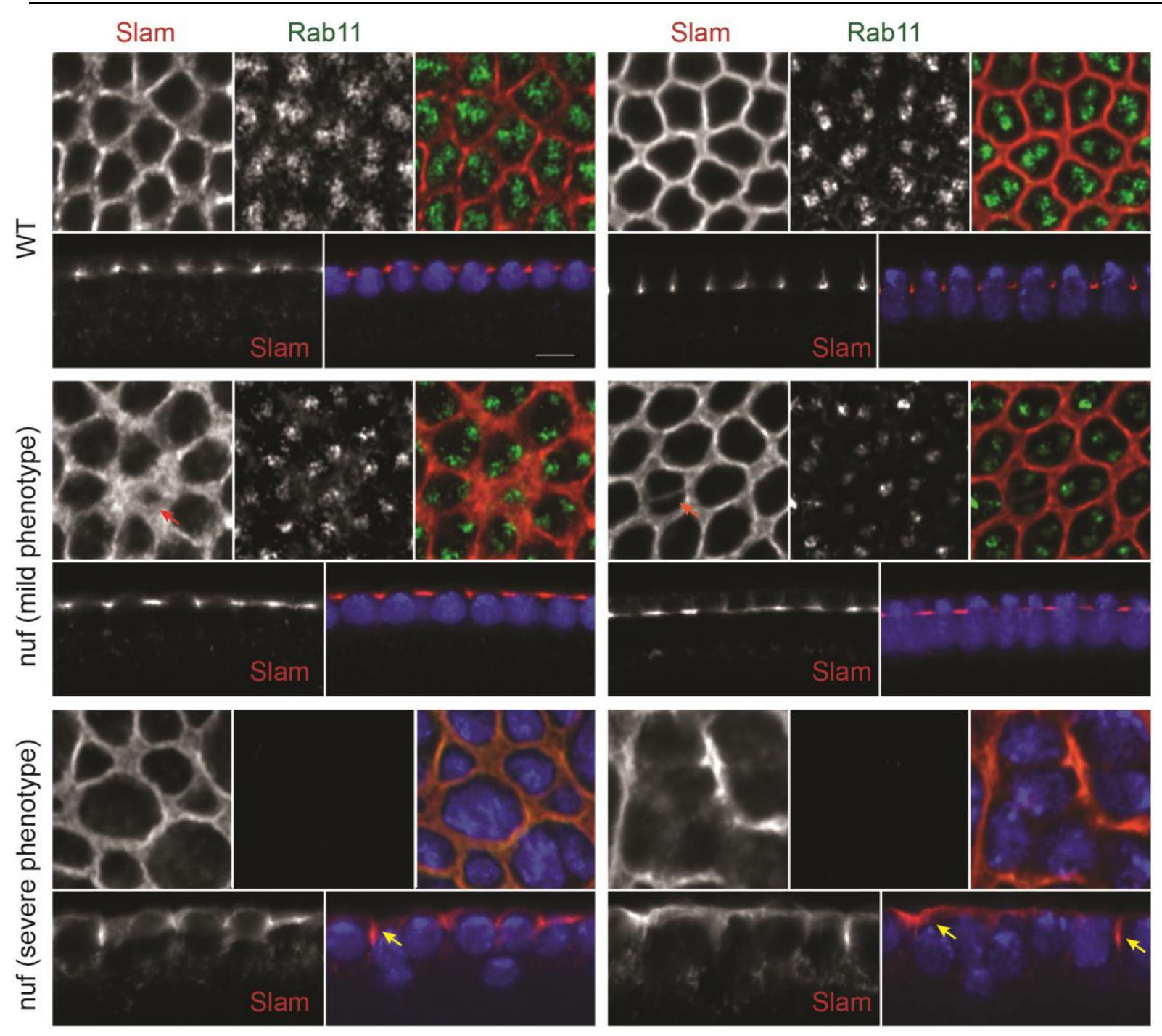

Fig 3.12 Slam mislocalisation corresponds to the severity of nuf phenotype. In mild nuf phenotypes, Slam is mislocalised to the cortex in regions of nuclear fallout (Red arrow). Occasionally, an ectopic furrow between the two centrosomes is formed due to greater centrosomal separation in nuf embryos (Orange arrow). In severe nuf phenotypes, Slam is mislocalised to the cortex and the lateral domain of the furrow (Yellow arrows). Scale bar $10 \mu \mathrm{m}$.

The mislocalisation of Slam was found even in situations where Dlg was not spread into the basal domain, indicating an intact membrane polarity. This suggests that Slam mislocalisation was the cause and not a consequence of membrane polarity disruption.

To further examine the role of nuf in the restriction of Slam to the furrow canal, we observed GFPslam dynamics in nuf embryos (Fig 3.11B). In wild-type GFPslam embryos, we observed that at the onset of cellularisation, GFPslam was found around the centrosomal exclusion area at the prospective site of invagination. This state, however, persisted only briefly as GFPslam was seen to arrange itself in a pseudo- 
hexagonal array in a few minutes. In nuf embryos, these accumulations persisted for a much longer time and sometimes failed to resolve into the pseudo-hexagonal array. Eventually, nuclei begin to fall out and Slam accumulates at the cortex. Together, these data suggest that a functional recycling endosome is necessary for the restriction of Slam to the basal domain.

We found that the extent of Slam mislocalisation in nuf embryos was dependent upon the severity of the nuf phenotype. The severity of phenotype was evident by abnormal nuclear morphology and loss of Rab11 staining (Fig 3.12). Another interesting observation was that due to nuclear fallout and spreading of the neighbouring nuclei, the centrosomes were found more apart from each other as compared to the wild-type (as indicated by Rab11 staining). Due to this, occasionally an extra furrow was formed between the centrosomes (Fig 3.12, orange arrow), further
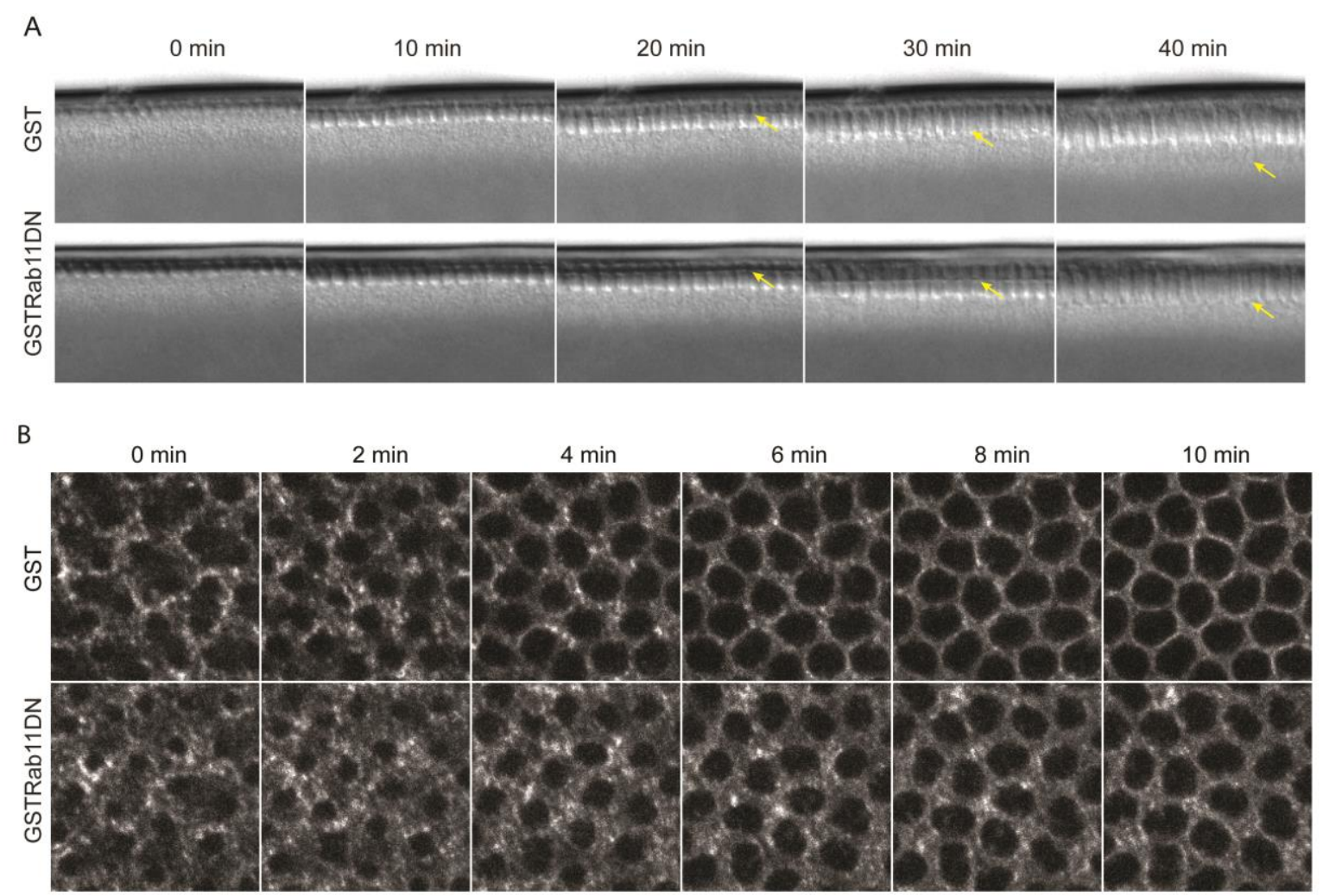

Fig 3.13 Interference with Rab11 function in the embryo causes delay in Slam restriction to the furrow canal. (A) Images from time lapse recordings of wild type embryos injected with purified GST or GST-Rab11S25N (dominant negative allele) protein. Arrows point to the cellularisation front. (B) Fluorescent images from time lapse recording of embryo expressing GFPslam and injected with GST (10 embryos) or Rab11S25N (at least 3 out of 10 embryos showed delayed Slam restriction). 
confirming our earlier conclusion that centrosomes determined the site of membrane invagination.

Since Nuf and Rab11 form a physical complex and are mutually dependent on their localisation (Riggs et al., 2003), we tested whether GFPslam restriction at the furrow canal is affected also when Rab11 function is disturbed. To accomplish this, we injected a dominant negative Rab11 allele, Rab11S25N (Serine 25 mutated to Asparagine) in GFPslam embryos and followed it by live imaging. It has been reported earlier that injection of purified Rab11S25N protein delays furrow invagination (Pelissier et al., 2003). About $40 \%$ of the injected embryos $(n=5)$ showed delayed furrow invagination (Fig 3.13 A) and GFPslam restriction at the furrow canal was also delayed by several minutes (Fig $3.13 \mathrm{~B}$ ).

\subsection{Dynamics of Slam protein, mobility and life-time}

\subsubsection{A fraction of Slam is membrane-associated}

Structured Illumination Microscopy has revealed that Slam is found at the membrane in a punctate form rather than being evenly distributed (Fig $3.14 \mathrm{~A}$ ). Previously, it has been shown that Slam is present in both cytoplasmic and membrane fractions in the embryo (Lecuit et al., 2002). We confirmed this result using an antibody against endogenous Slam as opposed to the anti-HA antibody which was used by Lecuit and colleagues. Additionally, we found out that Slam can be solubilised from the membranes using a high-salt membrane extraction which means that Slam is merely associated with the membrane and is not a transmembrane protein (Fig 3.14 B). This is in accordance with the fact that domain prediction of Slam does not reveal any transmembrane-domains in the protein. 
A

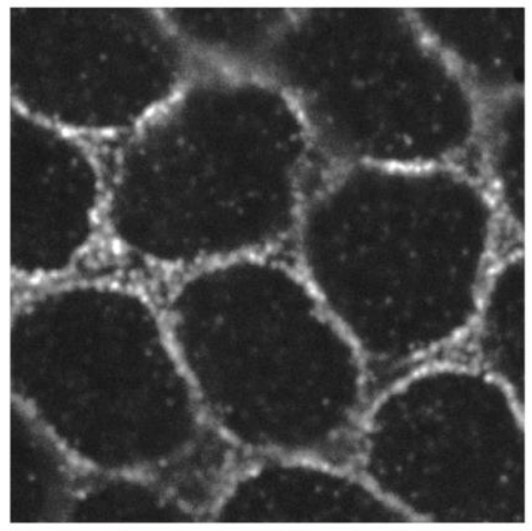

B

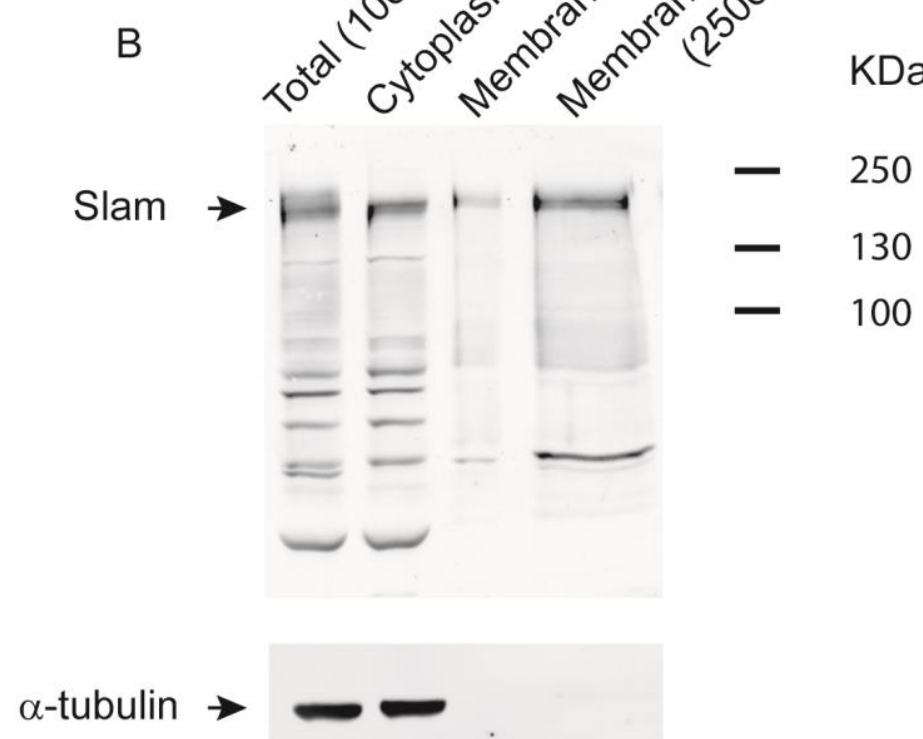

Fig 3.14 Slam is a membrane-associated protein. (A) Structured-illumination microscopy (SIM) reveals that Slam is distributed at the furrow canal in a punctate manner (Imaging carried out by Olaf Schulz) (B) Fractionation of WT embryos into cytoplasmic and membrane fractions was carried out and the membrane fraction solubilised with $1 \mathrm{M} \mathrm{NaCl}$. Slam is solubilised by the high-salt treatment indicating that it is a membrane-associated protein.

\subsubsection{Slam protein is stable during cellularisation}

We asked whether Slam protein turnover is fast during cellularisation or whether the protein is stable. To determine the half-life time of Slam protein, we first utilised embryos expressing Eos-slam. Eos is a photoactivatable fluorescent protein that undergoes an irreversible photoconversion from green emission peak to a red emission peak when exposed to a wavelength of $390 \mathrm{~nm}$ (Wiedenmann et al., 2004). We photoconverted an area of an embryo expressing Eos-slam during cycle 14 and measured fluorescence decay over time (Fig 3.16 A). However, it was realised that this was not an optimal system because a) the photoconversion was not efficient and it was not possible to attain a $100 \%$ conversion b) Photoconversion of a large area was not feasible and hence the effects of diffusion masked the actual degradation rate of Slam. 
Therefore, we switched to another assay to calculate the half-life time of Slam. Cycloheximide is a potent translation inhibitor and injecting it into syncitial Drosophila embryos blocks cell cycle in the $\mathrm{G}_{2}$ phase (Zalokar and Erk, 1976; Edgar and Schubiger, 1986). To first determine the effect of blocking protein translation in different stages of cycle 13 and cycle 14, cycloheximide was injected in different stages of embryos expressing GFPslam (Fig 3.15). As a positive control, we injected cycloheximide in histone-RFP embryos. It was seen that injection of cycloheximide prevented the embryo from entering the next mitosis as reported earlier (Fig 3.15 A). Injections were carried out ten minutes before the stage of cellularisation we intended to perturb. When cycloheximide was injected during anaphase of cycle 13 (Fig 3.15 B) to block protein synthesis at early cycle 14 , cellularisation was severely affected. Membrane morphology was disturbed and the embryos displayed loss of membrane

A
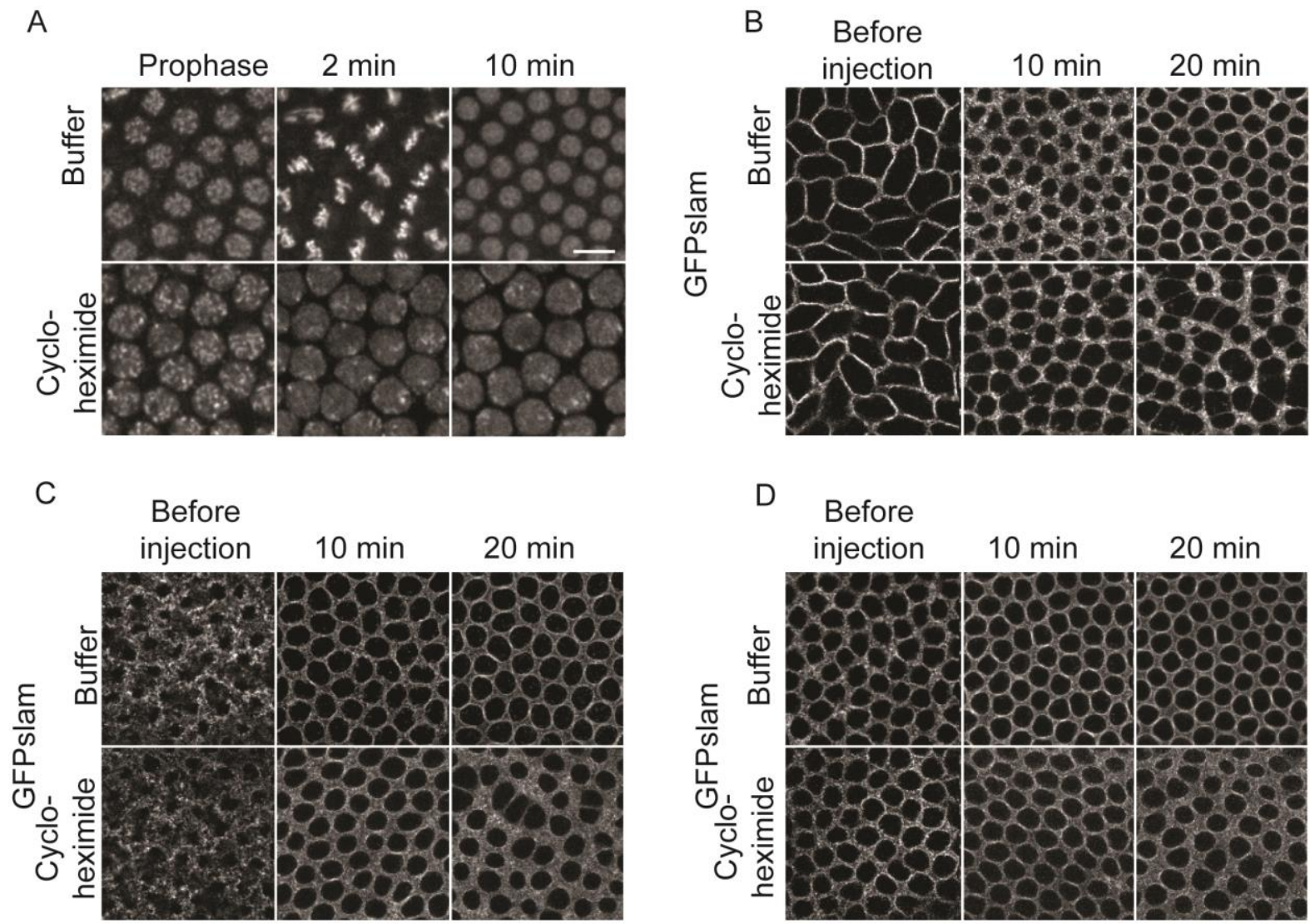

Fig 3.15 Effect of blocking protein translation in different stages of embryo. (A) Cycloheximide injected in syncytial embryo expressing his-RFP; the embryo is unable to finish mitosis. (B,C,D) Cycloheximide injected during Anaphase of cycle 13, onset of cycle 14 and mid-cycle 14 respectively. Scale bar $10 \mu \mathrm{m}$. 
between cells. The loss of membrane was not biased towards 'old' or the 'new' membranes, suggesting that protein synthesis during early cellularisation is essential for the formation of the new membrane as well as the maintenance of the old membrane. The severity of phenotype was reduced when the injection was carried out at the onset of cellularisation (to induce protein synthesis block at mid-cellularisation; Fig $3.15 \mathrm{C}$ ) and was even milder when injected during mid-cellularisation (to induce protein synthesis block at late-cellularisation; Fig 3.15 D). The loss of membranes between the cells depended on how early the injection was carried out. In the later stages, cycloheximide only induced thickening of membrane but loss of membranes was not noticed. This implies that synthesis of new proteins is most crucial at the onset of cellularisation and once the embryo starts cellularising, the need for new protein synthesis is diminished.

A

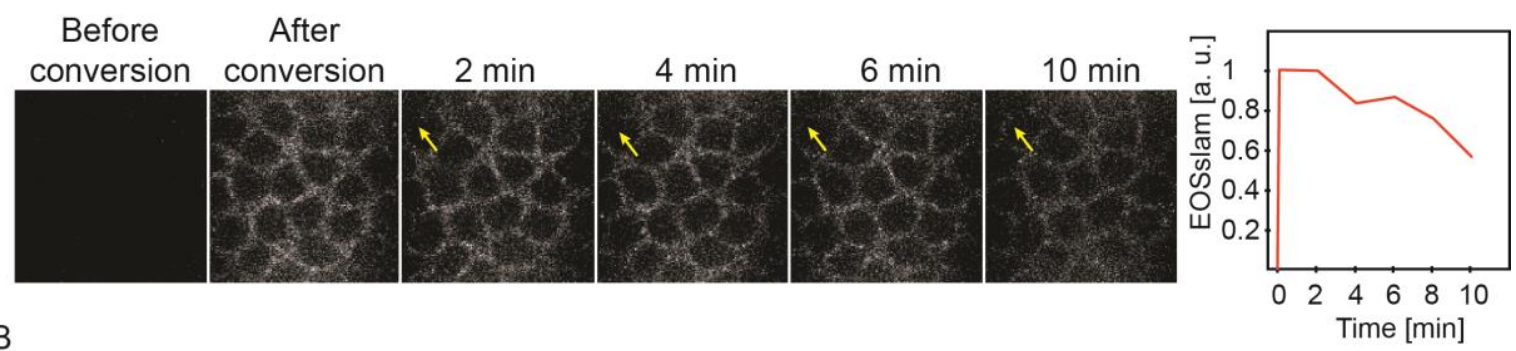

B

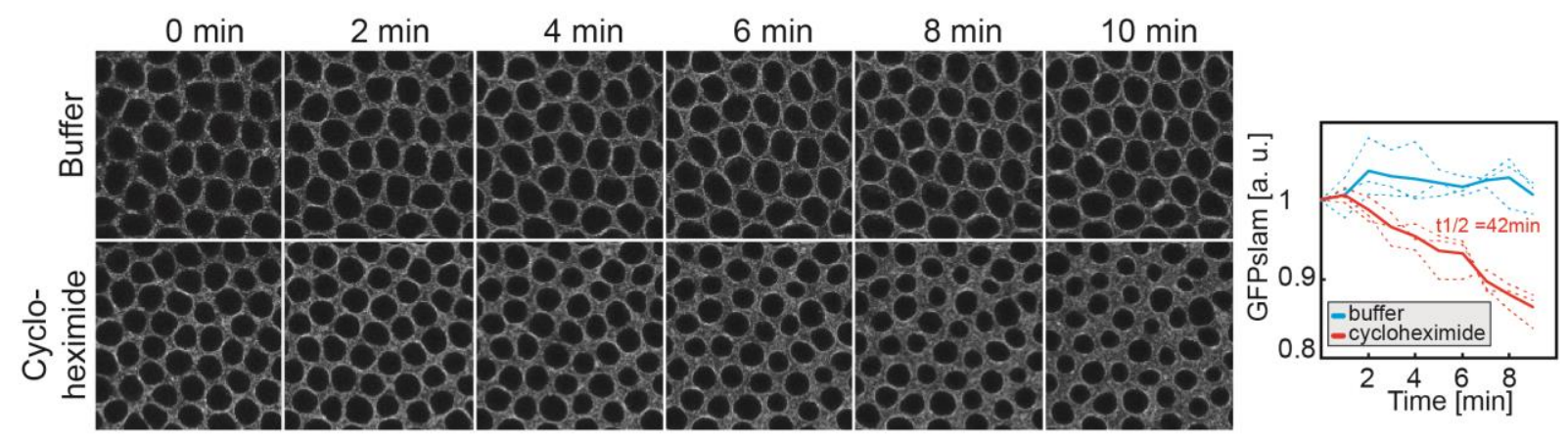

Fig 3.16 Slam protein is stable during cellularisation. (A) EOSslam was converted from its green emission to red emission peak and was followed using time-lapse recordings. Yellow arrows point to the diffusion of EOSslam signal thus interfering with protein half-life calculation. (B) GFPslam embryos were injected with buffer or Cycloheximide and the reduction of fluorescence signal was followed to calculate the protein half-life.

We measured the fluorescence of embryos injected with cycloheximide during mid-cellularisation. Half-life of Slam was determined by using the half-life equation:- 


$$
t_{1 / 2}=t \times \frac{\ln (2)}{\ln \left(\frac{N_{o}}{N_{t}}\right)}
$$

where $t_{1 / 2}=$ half-life time, $\mathrm{t}=$ time, $N_{o}=$ initial fluorescence and $N_{t}=$ final fluorescence. The half-life time of Slam is about 42 min (coefficient of determination, $R^{2}=\sim 97 \%$ ), which is almost $70 \%$ of the duration of cellularisation process. This indicates that Slam remains stable without being degraded for most part of cellularisation.

\subsubsection{Mobility of Slam is independent of new translation}

slam protein and mRNA colocalise with each other at the furrow canal (Wenzl et al., 2010). Therefore it is plausible that the mRNA is necessary for localised translation at the furrow canal or anchoring of the protein at the furrow canal or both. We asked whether preventing new synthesis of Slam would affect the mobility of Slam at the furrow canal. To test this, we carried out FRAP experiments to check for any changes in Slam mobility in mid to late cellularisation, in the absence of new translation (Fig 3.17). Cycloheximide was injected in embryos expressing GFP-slam and bleaching of a defined area was carried out after $10 \mathrm{~min}$. It was found that the mobility at this stage was unaffected in cycloheximide-injected embryos. It implies that impairing new translation has no effect on the mobility of Slam during mid to late cellularisation.

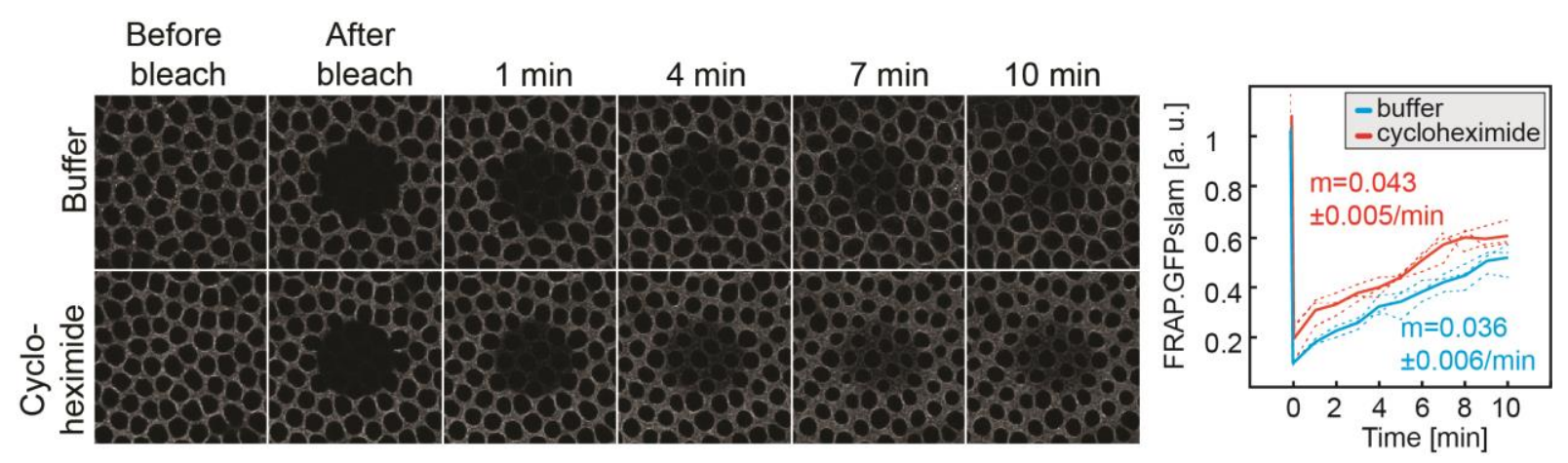

Fig 3.17 Mobility of Slam during cellularisation is independent of new translation. FRAP was carried out during cellularisation in embryos expressing GFPslam and injected with buffer or Cycloheximide. Fluorescence recovery of the bleached region was measured over time. 
This experiment, however, does not rule out the possibility that Slam mobility could be affected at the onset of cellularisation. FRAP for GFPslam at the onset is not feasible since injecting cycloheximide in cycle 13 to attain a protein synthesis block at the onset of cellularisation leads to a block of cellularisation. Therefore, it is only possible to induce the phenotype after the onset during early cellularisation. However, even at this stage rapid loss of membranes and change in membrane morphology makes the analysis difficult.

\subsubsection{Mobility of Slam is not directly affected by the recycling endosome}

Slam shows a very unique behaviour in terms of mobility at the furrow canal. FRAP experiments have revealed that it shows a very stable association at the furrow canal during interphase of cell cycle 13 and 14 . However, at the onset of cellularisation, it becomes very mobile and exchanges very rapidly (Acharya et al., 2014; Dr. Philip Laupsien, $\mathrm{PhD}$ dissertation). This is in contrast with the behaviour of other furrow canal markers such as Amphiphysin and 4XPDZ-RFP (four PDZ domains of RhoGEF2 fused with RFP) which show a high mobility at all times. We asked whether this change in mobility of Slam is achieved gradually or whether it is a switch-like behaviour. To answer this, FRAP was carried out in GFPslam embryos at defined time-points and fluorescent recovery was observed. It was observed that during the onset of cellularisation, the rate of recovery was above or equal to $10 \%$ per minute whereas once the 'new furrow' was formed completely, the rate of recovery dropped to $3 \%$ per minute. The rate of recovery was determined by calculating the linear slope of fluorescent recovery per minute (Fig 3.18). No intermediate recovery rates were found, suggesting a switch-like change in Slam mobility once the 'new furrow' is formed.

Since Slam restriction to the furrow canal is dependent on the recycling endosome, we looked at possible changes in Slam mobility in nuf embryos (Fig 3.19). Out of the five embryos that we observed, three showed normal behaviour of Slam, which can be attributed to the variable phenotype of nuf mutants. Two embryos, however, showed slightly increased mobility (about 6\% per minute) (Fig $3.19 \mathrm{C}$ ). Apart from the slight increase in mobility, it was observed that the unbleached region in these embryos showed an increase in fluorescence, which was never observed in wild-type 


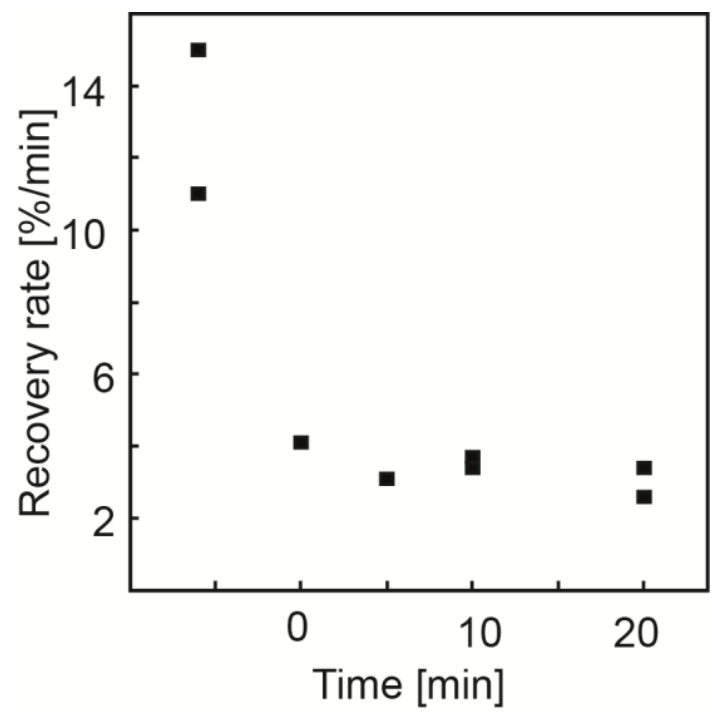

Fig 3.18 Slam is stably associated to the membrane during interphase 13 and 14 and switches to a high-mobility state at the onset of cellularisation. FRAP was carried out at different stages of cellularisation. Time point ' 0 ' is immediately after the onset of cellularisation right after the formation of the new furrow. The fluorescence recovery rate was calculated by measuring the linear slope of fluorescence recovery. The behaviour of Slam changes in a switch-like manner from a very highly mobile state before the onset of cellularisation to a stable, low mobility state after the onset of cellularisation.
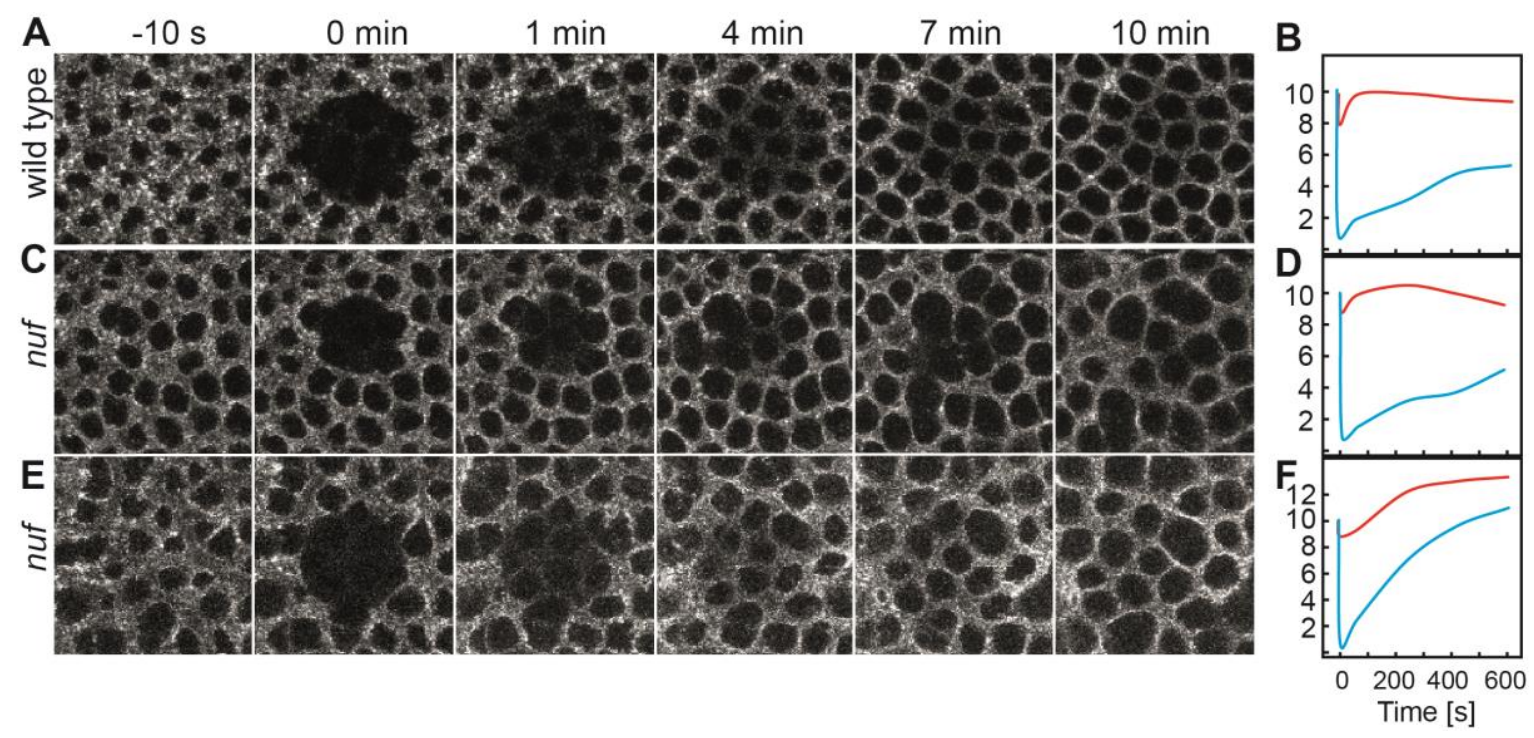

Fig 3.19 Slam mobility is slightly increased in a subset of nuf embryos. (A,B) GFPslam FRAP in WT background (C,D) GFPslam FRAP in nuf embryos showing recovery like WT (E,F) GFPslam FRAP in nuf embroys showing increased recovery and excessive GFPslam accumulation in the unbleached region.

embryos. This can be explained by the fact that Slam spreads into the lateral membrane due to decreased restriction at the furrow canal in nuf mutants. Thus, less 
spacial constraints allow more Slam to accumulate at/near the furrow canal, causing increase in fluorescence. To further investigate whether the change in mobility of Slam during cellularisation is dependent on the recycling endosome, we carried out FRAP experiment in shibire mutant embryos. FRAP of GFPslam during mid-cellularisation showed no difference in wild-type and shibire mutants (Fig 3.20). Therefore the slight increase in mobility of Slam in nuf embryos could be the due to the mistargeted Slam population at the apical and lateral regions being more mobile. Taken together, these data show that the recycling endosome plays a role in targeting Slam to the furrow canal and maintaining its spacial restriction but the mobility of Slam during cellularisation is not directly dependent on vesicular trafficking.
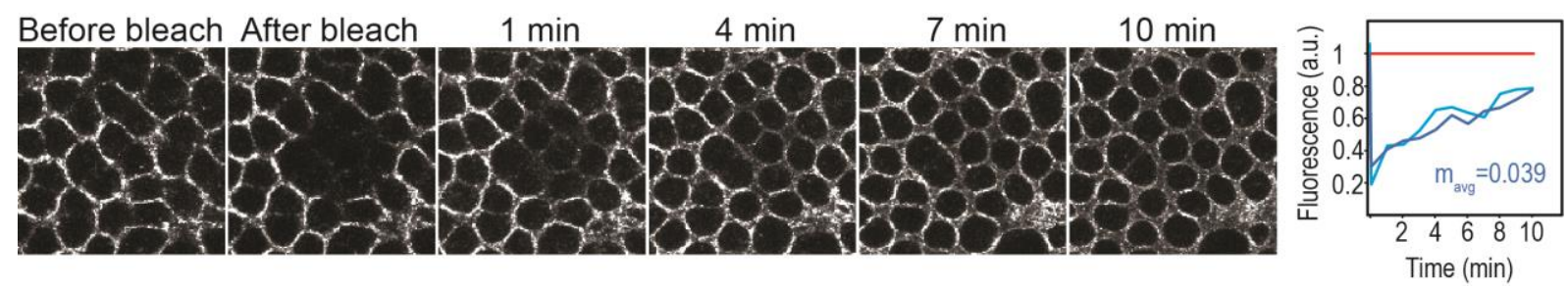

Fig 3.20 The mobility of Slam during cellularisation is not dependent on vesicular trafficking. FRAP was carried out during cellularisation in WT and shibire embryos expressing GFPslam. Fluorescence recovery of the 'old furrows' was measured over time.

\subsection{Role of Slam during cellularisation}

\subsubsection{Additional factors apart from Slam are needed for furrow specification}

Different alleles of slam were analysed to see whether the effect on furrow invagination is variable depending on the mutation (Fig 3.21). The alleles 35.16 and 20.89 are slamwaldo1 and slamwaldo2 respectively and have already been analysed and reported in Stein et al., 2002. They discovered that these alleles showed a germ cell migration defect in the presence of maternal slamwaldo contribution whereas $\mathrm{M}^{-} \mathrm{Z}^{-}$slamwaldo embryos failed to cellularise. We analysed the mutant alleles 9.35 and B4.1 in comparison with wild-type and embryos derived from slam germline clones (slam glc) which completely lack both maternal and zygotic Slam. Allele 9.35 has a point mutation at the $91^{\text {st }}$ amino acid where Phenylalanine is changed to Serine while allele B4.1 has a deletion from nucleotide 517 to 874 , causing a frame-shift that gives rise to a premature stop codon. Allele B4.1 produces a peptide product containing the first 173 
amino acids of Slam sequence fused to an additional 24 amino-acid long peptide that doesn't correspond to Slam.

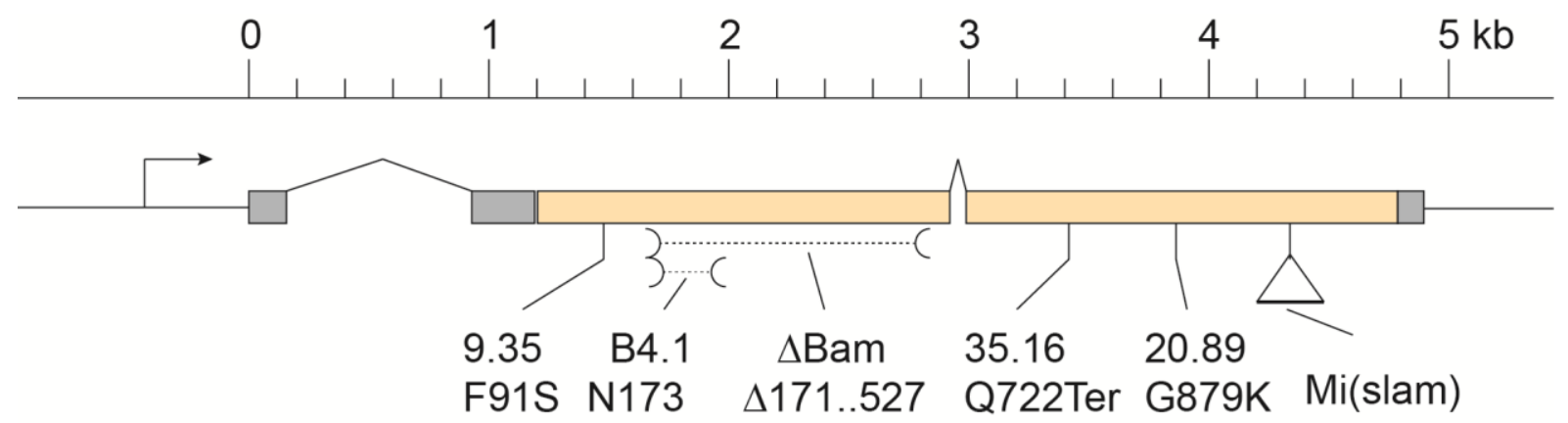

B4.1 nt (CDS) Delta 517..874

aa 1..172 + TSSSV TWQPS TPSSL HMPQI SRRR stop

Fig 3.21 Schematic representation of alleles of slam. The peptide sequence refers to the B4.1 allele that contains the first 172 amino acids of Slam and an additional 24 amino-acid long peptide, the first of which is $(T)$ is also present in the Slam sequence.

Live imaging of embryos was carried out using differential interference contrast (DIC) microscopy and the furrow invagination was compared between the alleles (Fig 3.22). It was found that furrow invagination in allele 9.35 was largely unaffected, though in some cases it was slightly delayed. In allele B4.1, furrow was formed and also invaginated, though only to a certain extent. Later nuclear morphology was disrupted and the embryos failed to cellularise. In comparison, the most severe phenotype was seen in slam glc embryos where there was no furrow invagination (Fig 3.22 ; Dr. Philip Laupsien, PhD dissertation). It is known that Slam is necessary, at least in part, for furrow specification (Lecuit et al., 2002). However, a vague furrow seemed to be specified despite Slam's absence (Fig 3.22, slam glc panel). When slam glc embryos were stained for various furrow markers, it was found that F-actin, Dia, Nullo were deposited at the furrow site, suggesting that there are additional signals for the specification of furrow apart from Slam (Acharya et al., 2014; Dr. Philip Laupsien, PhD dissertation).

\subsection{1 nullo and slam together control the specification of the cleavage furrow}

As mentioned earlier, furrow specification takes place even in the absence of slam. 


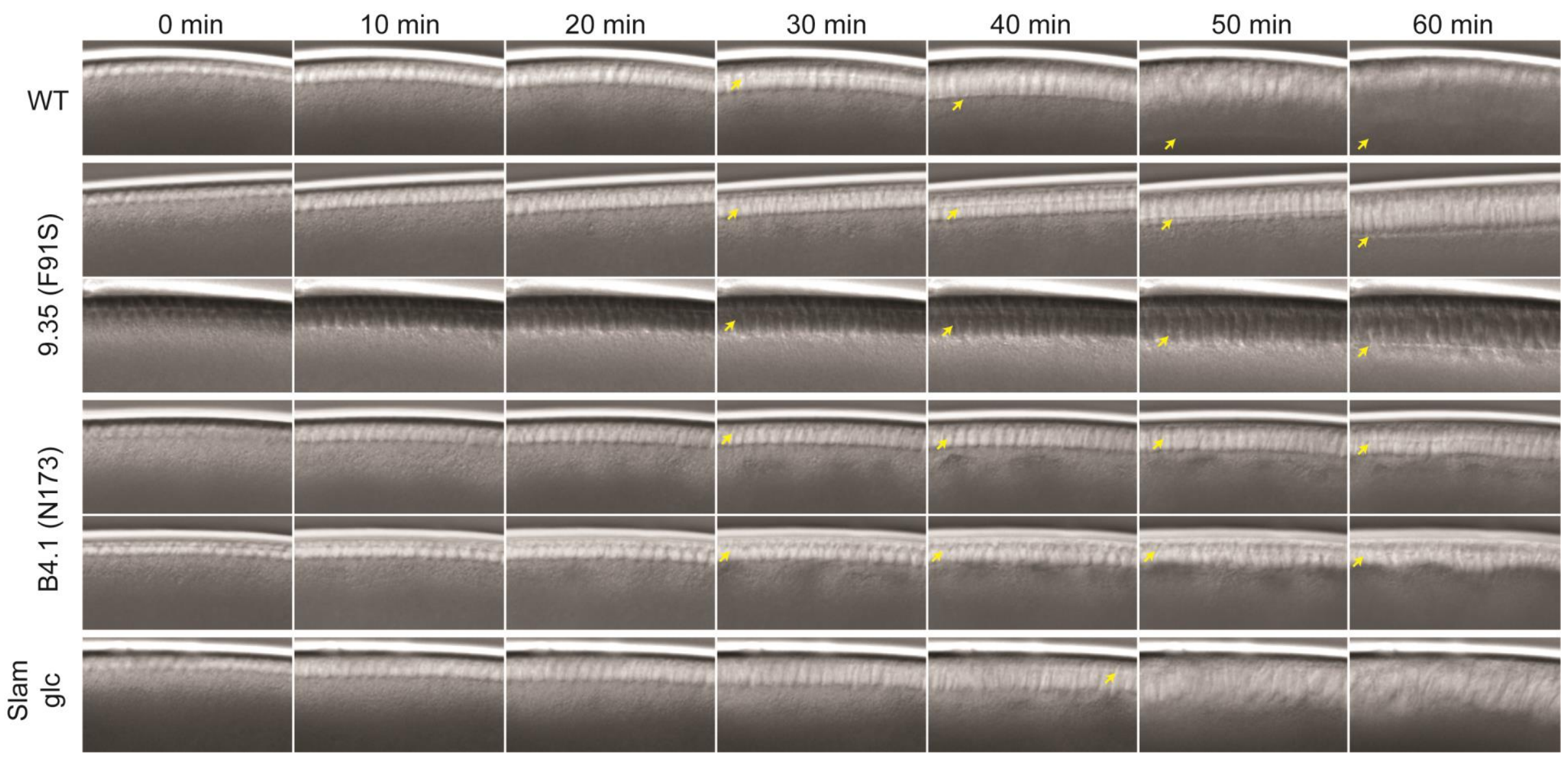

Fig 3.22 Furrow specification requires factors additional to slam. Time-lapse movies of embryos of different slam alleles were obtained by Differential Interference Contrast (DIC) microscopy. The yellow arrows mark the invaginating furrow. Allele 9.35 is a weak allele, usually showing a normal cellularisation but sometimes showing a delay in furrow invagination. Allele B4.1 shows invagination until about half of the length of the nuclei after which it fails to invaginate further. Slam glc (germline-clones) lack both maternal and zygotic Slam and fail to cellularise. However, the specification of a regressed furrow is visible. Yellow arrow represents the cellularisation front. 
This might either mean that slam is not involved in furrow specification or that there are additional redundant factors that are responsible for furrow specification. The zygotic gene nullo was a likely candidate that could act redundantly to slam because nullo has been shown to act redundantly to RhoGEF2 (Grosshans et al., 2005) while localisation of RhoGEF2 is dependent on slam (Wenzl et al., 2010). To test this, we generated embryos lacking zygotic nullo and both maternal and zygotic slam. The furrow was visualised by Dia staining. We found that in nullo;slam double mutants, Dia formed large accumulations in contrast to the single mutants where one could see a proper furrow structure (Fig 3.23). This shows that nullo and slam act redundant to each other to specify the furrow canal.
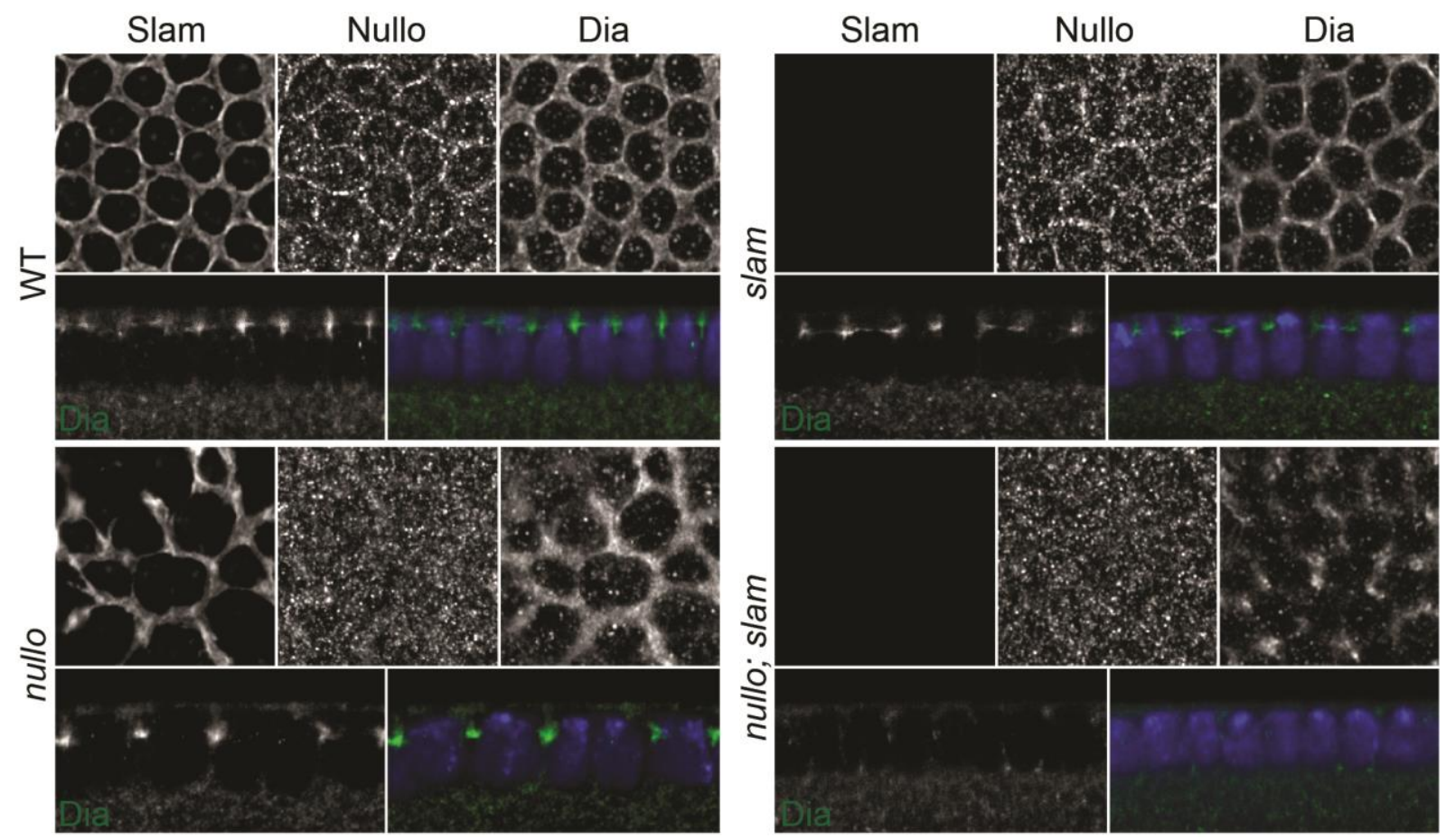

Fig 3.23 nullo and slam together control the specification of the furrow. Embryos from nullo heterozygous females with slam germline-clones crossed with slam heterozygous males. Genotype of embryos was determined by staining for Slam and Nullo. The furrow array was visualised by Dia (green) staining. 


\subsection{2 spire - an interactor of slam in yeast two-hybrid screen}

spire was identified as an interactor of slam in a yeast two-hybrid screen (Hybrigenics services; Dr. Philip Laupsien, PhD dissertation). Full-length Slam as well as its N-terminal fragment (amino acid 1 to 657) was found to interact with the $\mathrm{WH} 2$ domain (actin-nucleation domain) of Spire (Fig 3.24). Spire is a maternal-effect gene that is required for proper dorso-ventral and antero-posterior axis determination of the embryo. spire mutant oocytes display premature cytoplasmic streaming and the embryos lack pole cells (Theurkauf, 1994). Role of spire in cellularisation hadn't been explored yet and therefore we set out to characterise spire mutant phenotype in the embryos in further detail.

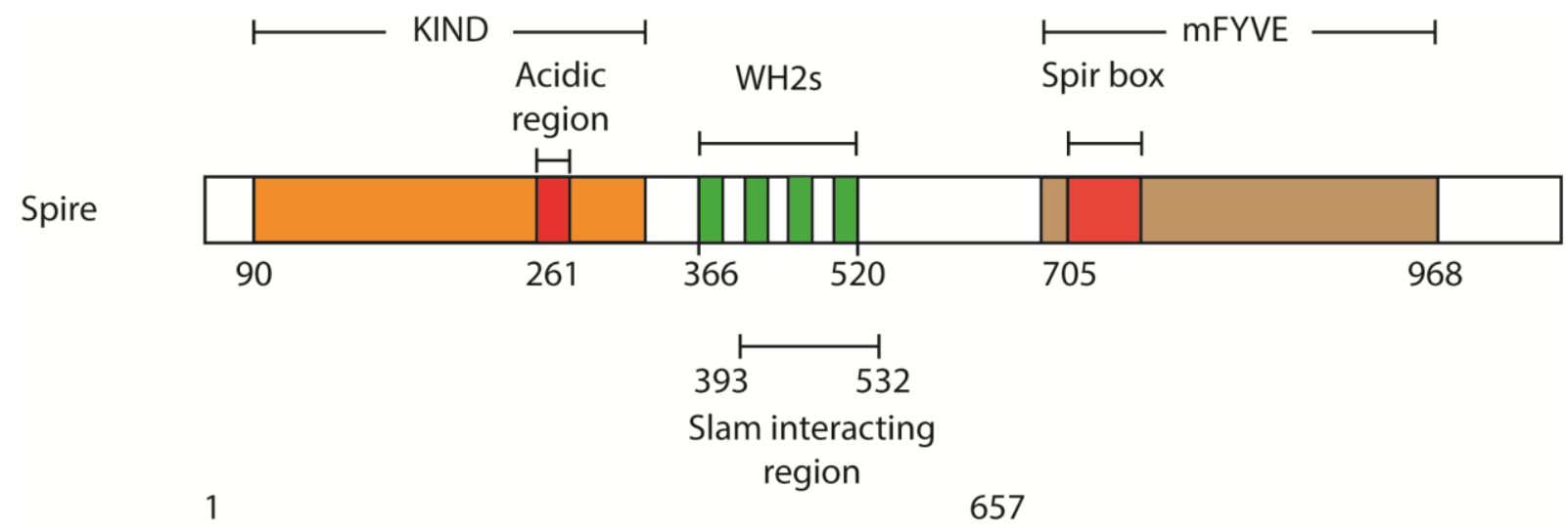

Slam

Fig 3.24 Schematic representation of interaction of Slam and Spire in a Yeast two-hybrid screen. The N-terminal part of Slam interacts with the WH2 (actin-binding) domains of the Spire protein. (Representation of Spire protein modified from Vizcarra et al., 2011).

We generated spire transheterozygote embryos (spir1/Df(2L)Exel6046 embryos) and analysed the phenotype using Differential Interference Contrast microscopy. None of the embryos displayed a cellularisation defect though many embryos had a cell cycle defect where either whole or part of the embryo would undergo an extra cell cycle, leading to a delay in membrane invagination in that region. We refer to this phenotype as the 'cell cycle phenotype'. Furthermore, we analysed a number of so far uncharacterised mutant alleles of spire. Some of them displayed a 'cellularisation phenotype' where furrow was formed and also invaginated but failed to complete the invagination. 


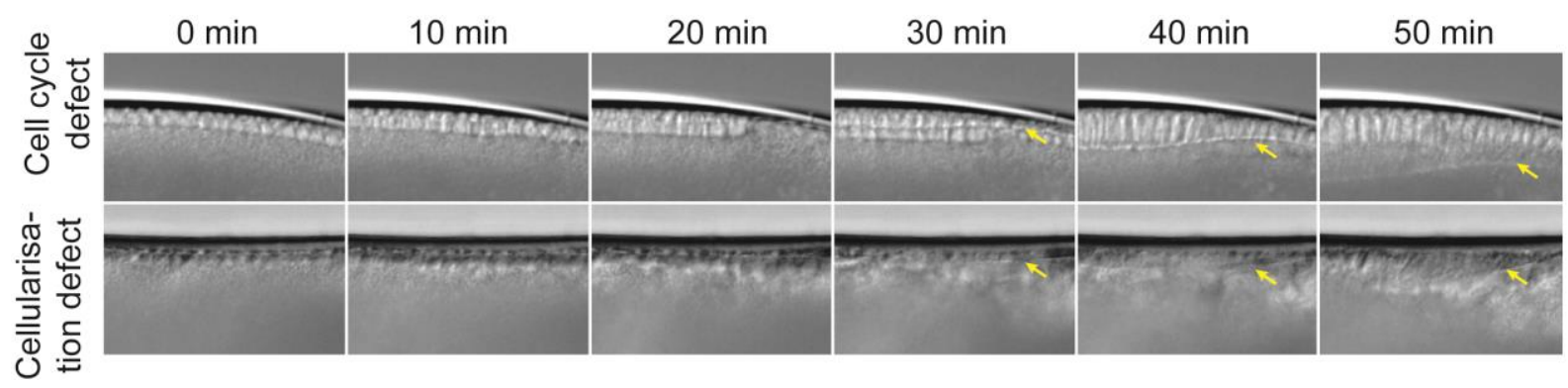

Fig 3.25 Spire alleles show cell-cycle defects and occasional cellularisation defects. Time-lapse movies of embryos of different spire alleles were obtained by Differential Interference Contrast (DIC) microscopy. The shown examples are representatives of the cell-cycle defect and the cellularisation defect.

Table 3.1 Penetrance of cell-cycle and cellularisation defects in different spire alleles

\begin{tabular}{|l|l|c|}
\hline \multicolumn{1}{|c|}{ Allele } & \multicolumn{1}{|c|}{ Phenotype } & $\mathrm{n}$ \\
\hline spir $/$ Df(2L)Exel6046 & $60 \%$ cell cycle defect & 10 \\
\hline spir ${ }^{2 L-62-29}$ & $33.3 \%$ cell cycle defect & 6 \\
\hline spir $^{2 L-75-28}$ & $7.7 \%$ cell cycle defect & 13 \\
\hline spir $^{2 L-133-31}$ & $33.3 \%$ cell cycle defect & 12 \\
\hline spir $^{2 L-146-30}$ & $\begin{array}{l}7 \% \text { cellularisation defect } \\
7 \% \text { cell cycle defect }\end{array}$ & 14 \\
\hline spir $^{2 L-210-2}$ & $\begin{array}{l}44.4 \% \text { cellularisation defect } \\
11 \% \text { cell cycle defect }\end{array}$ & 9 \\
\hline spir $^{2 L-216-18}$ & $30 \%$ cell cycle defect & 10 \\
\hline spir $^{2 L-244-35}$ & $\begin{array}{l}21 \% \text { cell cycle defect } \\
21 \% \text { cellularisation defect }\end{array}$ & 14 \\
\hline
\end{tabular}

spir transheterozygotes embryos (spir/1/Df(2L)Exel6046) display only cell-cycle defects. Cellularisation defect is most penetrant in the temperature-sensitive allele of spire (spir $\left.{ }^{2 \mathrm{~L}-210-2}\right) \cdot \mathrm{n}=$ number of embryos observed. 
Typical examples of the 'cell cycle phenotype' and cellularisation phenotype' have been depicted in Fig 3.25. The percentage of embryos displaying these phenotypes in the alleles analysed has been mentioned in Table 3.1.

Next, we generated an antibody against the KIND domain of Spire. KIND domain was purified using an N-terminal His tag under denaturing conditions (Fig 3.26 A) Antibodies were generated in Guinea Pig and Rabbit. Affinity purification of the antibodies was carried out and the purified antibodies were used for western blot and immunostaining. The results from the antibodies derived from guinea pig have been shown here. Western blot against KIND in staged embryos (1.5 to 2.5 hours) displayed several missing bands in the spir transheterozygote embryos when compared to wild-

A

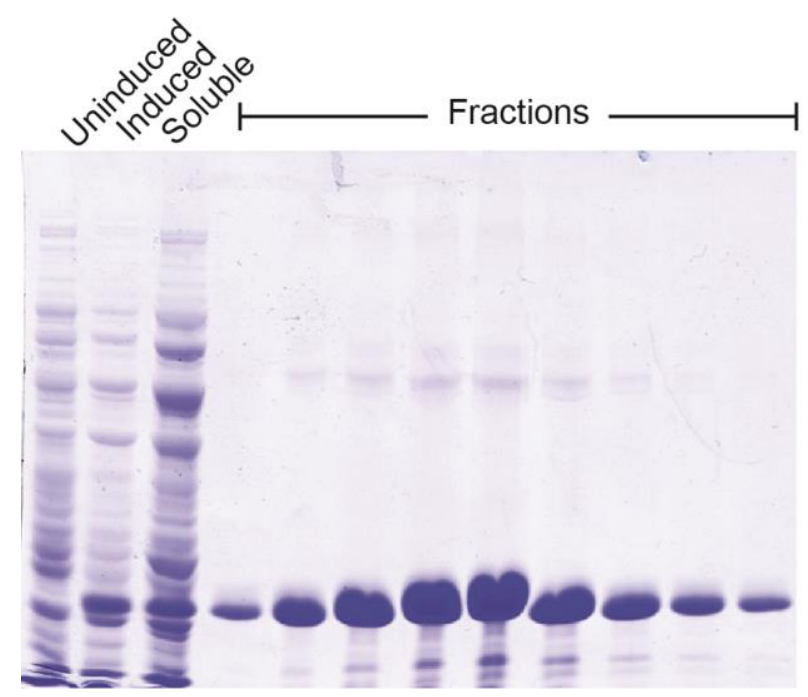

B

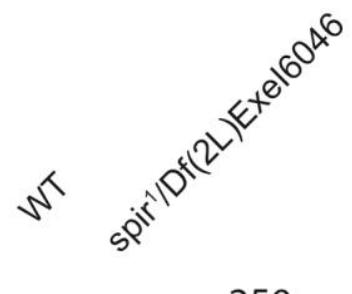

$-250$
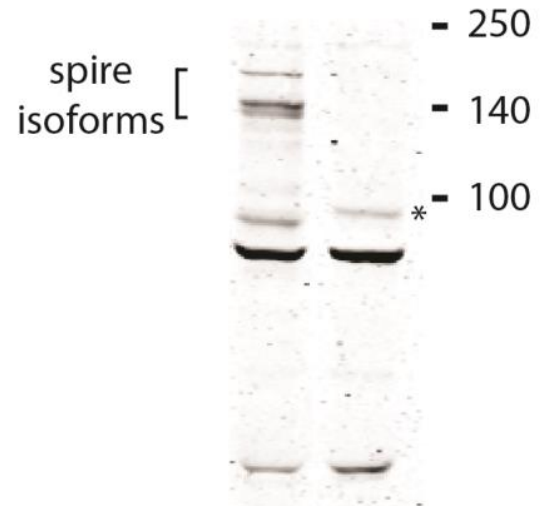

Fig 3.26 Spire antibody against the KIND domain detects several isoforms of Spire. (A) The KINDdomain was purified under denaturing conditions using a GST tag at the N-terminal part. (B) Western blot using the affinity-purified antibody developed against the KIND domain of Spire in WT and Spire transheterozygote embryos.

type. There was an additional band just below $100 \mathrm{kDa}$ (Fig 3.26 B, marked by *) which does not correspond to the size of the truncated Spir fragment arising from the spir ${ }^{1}$ allele. It is unclear as to what this band represents. There were also additional background bands suggesting that the antibody is not entirely specific. 
Nevertheless, we utilised this antibody to carry out immunostaining in embryos to determine the localisation of Spire during cellularisation. A strong apical staining reminiscent of actin caps was noticed in cellularising embryos (Fig 3.27). However, we found a similar staining pattern in spir transheterozygote embryos (not shown). Since the spir ${ }^{1}$ allele leads to a truncated fragment that includes about $85 \%$ of the KIND domain, it is likely that this fragment is still detected in the transheterozygote embryos, provided the fragment still retains localisation ability. A complete null mutant of spir would clarify the correct localisation pattern of Spire in the embryos.

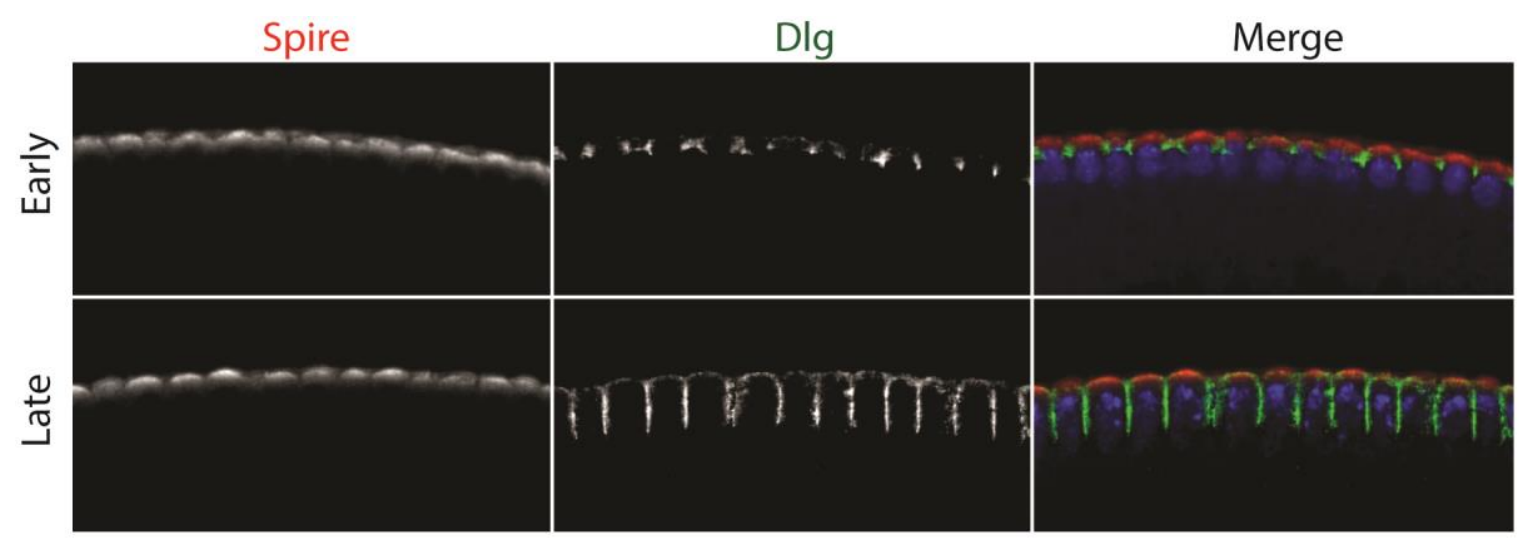

Fig 3.27 Immunostaining against Spire shows apical staining during cellularisation. WT embryos in early and late cellularisation stained with Spire (red) and Dlg (green). Spire accumulates at the apical domain of the cellularising embryos, in a pattern similar to Actin caps in the earlier cell cycles.

However, we did not proceed to generate a complete null of spir because we could not find a common phenotype between slam and spir. Cellularisation phenotype in spir was milder and significantly different from the slam phenotype and slam mutants do not display cell-cycle defects. Also the pole cell formation is normal in slam mutants. Therefore, we concluded that Spire might have a redundant role in cellularisation as the phenotype is neither fully penetrant, nor very severe.

\subsubsection{Other interactors of Slam}

In the above mentioned yeast two-hybrid screen, Slam was also found as an interactor. This could mean that Slam might form a dimer/oligomer. To test this, we carried out pulldown of GFPslam using GFP-binder magnetic beads from staged 
embryos. Endogenous Slam was not coprecipitated with it (Fig 3.28 A). Therefore we did not find any evidence of dimerisation of Slam.

In an attempt to discover more interactors of Slam, we planned to carry out pulldown of Slam by immunoprecipitation, followed by Mass Spectrometry. For this purpose, antibody against Slam raised in Rabbit was affinity purified (Fig 3.28 B). We were able to reduce the background via affinity purification, though there were still some unspecific bands detected. Endogenous Slam and GFPslam were immunoprecipitated using this antibody (Fig $3.28 \mathrm{C}$ ) and we could show that this method can be utilised in the future for large scale immunoprecipitation followed by mass spectrometry to obtain more interactors.

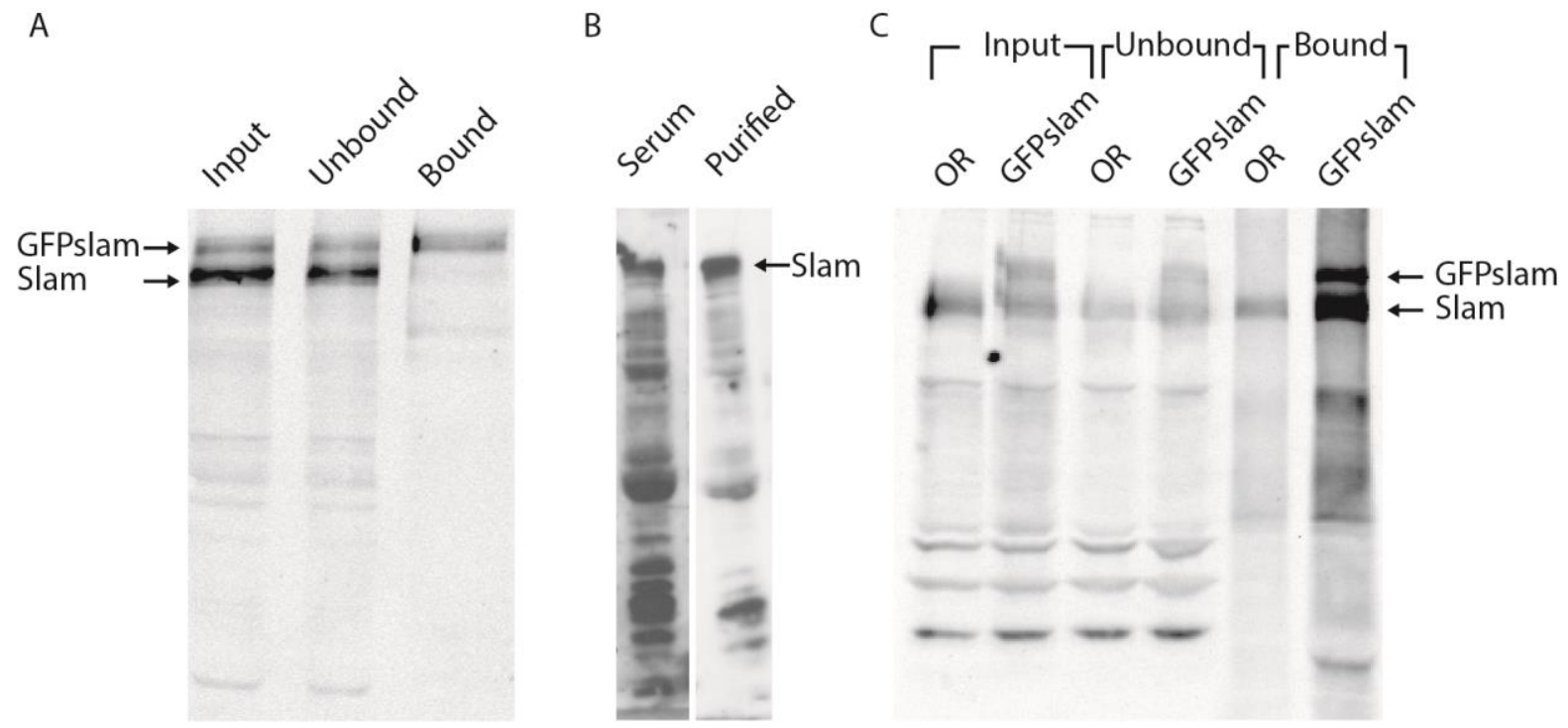

Fig 3.28 Pulldown of Slam and GFPslam. (A) GFPslam pulldown using Streptavidin-magnetic beads. Only GFPslam is detected in the bound fraction (Courtesy: Stephanie Gröning) (B) Affinity purification of rabbit anti-slam antibody (C) Immunoprecipitation using affinity-purified antibody can precipitate both GFPslam and endogenous Slam. Input and Unbound $\sim 10$ embryos/lane, Bound $\sim 500$ embroyos/lane.

\subsection{Slam protein properties and the RNP complex}

\subsubsection{Slam has a predicted structured $\mathrm{N}$-terminal half}

Slam is a non-conserved protein with no known functional domains. Secondary structure prediction of Slam shows that it has a highly disordered structure in the middle 
region from amino acid 450 to 850 (Fig 3.29). The $\mathrm{N}$-terminus and the $\mathrm{C}$-terminus on the other hand, contain more predicted ordered regions.

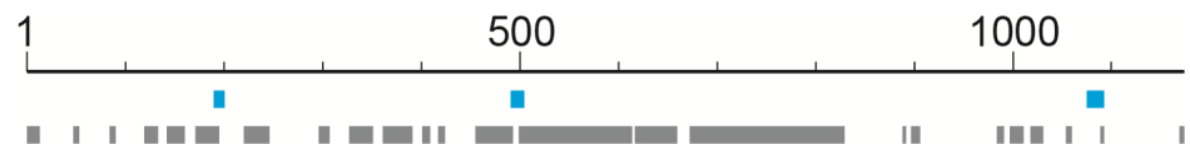

Fig 3.29 Schematic representation of disordered regions in Slam protein. The gray areas represent disordered regions. The blue regions show putative coiled-coil regions (Prof. Jörg Großhans).

\subsubsection{GFP tag at the N-terminus of Slam partially interferes with its function}

We determined whether the genomic GFPslam was able to rescue viability of flies (Section 2.2.3.7, paragraph 3). We could not obtain flies that contained only
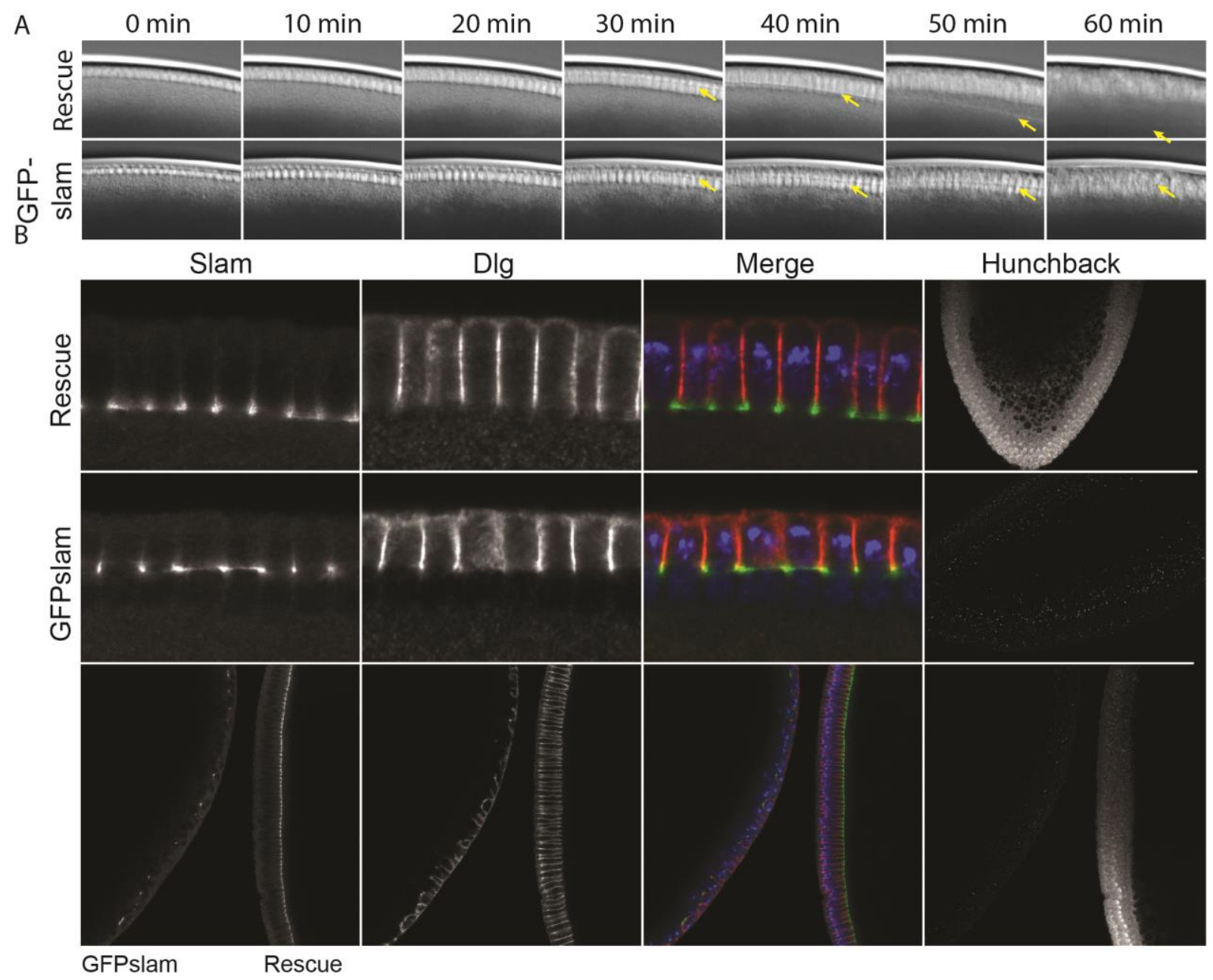

Fig. 3.30 GFP tag partially interferes with Slam function. (A) DIC microscopy showing development of rescued embryos and GFPslam embryos; yellow arrows indicate the cellularisation front (B) GFPslam embryos can proceed into cellularisation to a certain extent but unable to finish the process. 
GFPslam in the absence of endogenous slam. We further looked for rescue of the cellularisation phenotype (Section 2.2.3.7, paragraph 4) and found that embryos containing only GFPslam were able to cellularise to a large extent but were unable to complete cellularisation (Fig 3.30). This suggests that the N-terminal GFP tag causes interference with Slam function in some way.

To identify any difference in localisation behaviour between GFPslam and Slam, fractionation of embryos expressing both GFPslam and endogenous slam was carried out. It was found that the population of GFPslam at the membrane was lesser compared to endogenous Slam (Fig 3.31). This reduced localisation efficiency could explain the inability of GFPslam embryos to finish cellularisation.

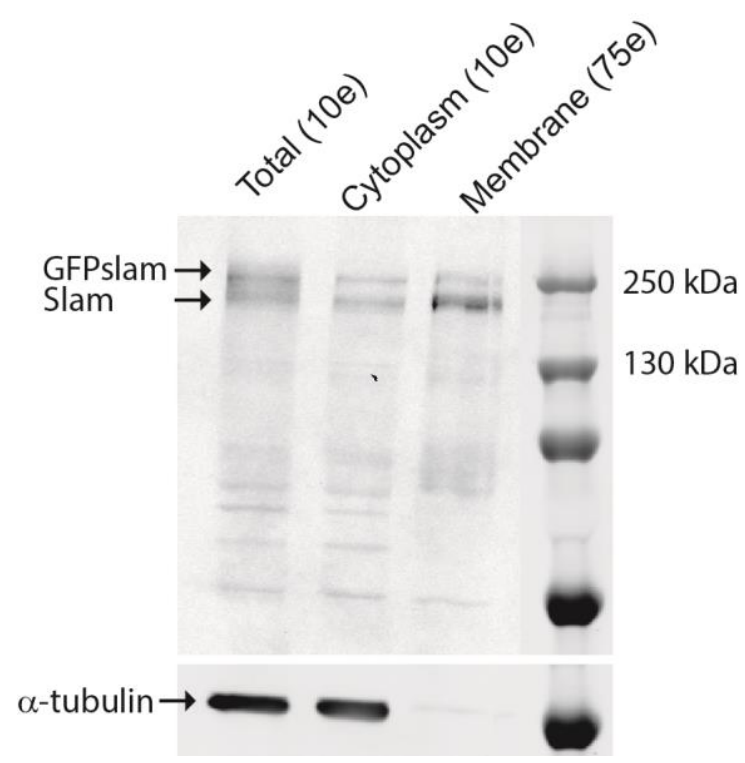

Fig 3.31 GFPslam is less efficiently bound to the membrane. Fractionation of staged embryos (1.5$2.5 \mathrm{hr}$ ) shows that the efficiency of GFPslam localisation is less in comparison to the endogenous Slam.

\subsubsection{Slam protein and slam mRNA are present in a complex}

It was shown earlier that Slam mRNA and protein colocalise at the furrow canal and basal particles (Wenzl et al., 2010). It was found in our lab that the protein and the mRNA are present in a complex. When Slam protein was immunopreciptated, the mRNA was coprecipitated with it (Fig 3.32; Dr. Shuling Yan, unpublished). We wanted to find out whether slam mRNA was enriched at the membrane when compared to a control mRNA. To determine this, fractionation of staged embryos was carried out and protein and mRNA were detected using western blot and qRT-PCR respectively (Fig 
3.33). As a reference we quantified actin mRNA. We found that actin mRNA is enriched in the cytoplasmic fraction ( $\sim 4$ folds) and slightly depleted in the membrane fraction. In contrast, slam mRNA was slightly enriched in the membrane fraction (at least $\sim 1.4$ fold, and on an average $\sim 3$ fold in three independent experiments). This shows that a large pool of slam mRNA is membrane-associated indicating that the mRNA could possess some non-coding functions. The variation in the qPCR $C_{t}$ numbers in different experiments (Table 3.2) is most likely due to loss of membranes during the fractionation procedure. Indeed the $C_{t}$ numbers of actin and slam mRNA correspond to each other in all experiments.

A

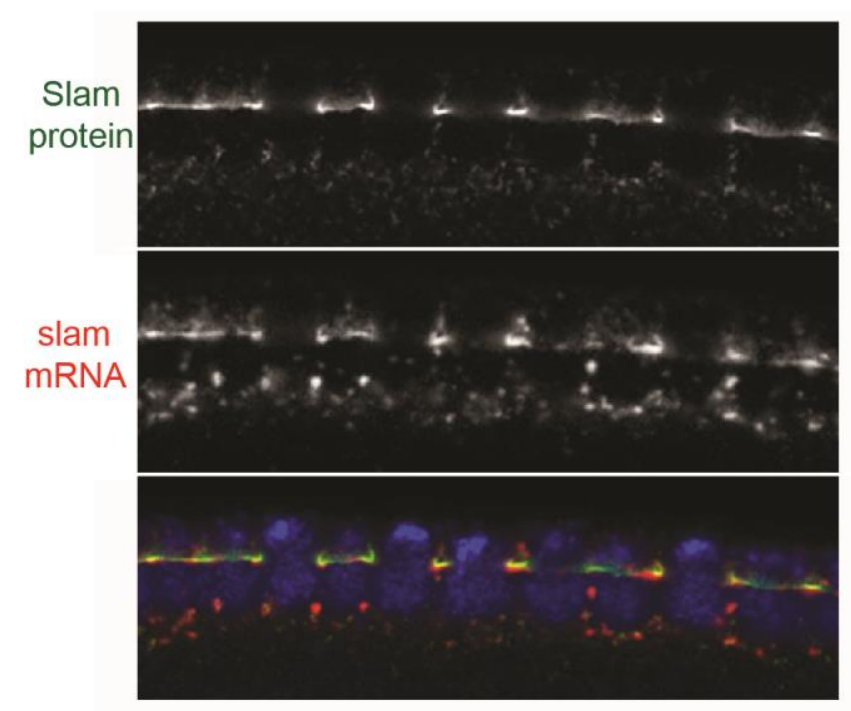

B
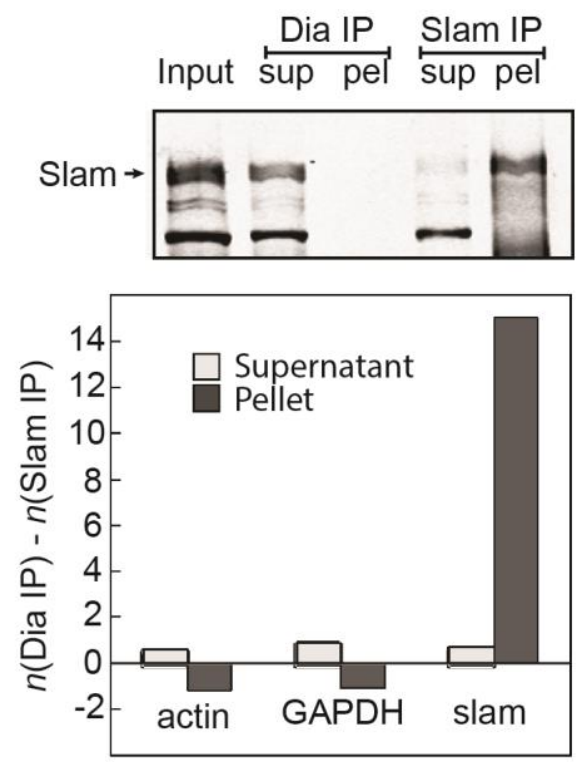

Fig 3.32 slam RNA and protein are present in a complex. (A) Slam mRNA (red) and protein (green) colocalise at the furrow canal and the basal particles. (B) Slam protein was immunoprecipitated and detected by a western bot and slam mRNA was detected by qRT-PCR. The mRNA was found to be enriched in the immunoprecipitated sample when compared to a control mRNA. (Experiments carried out by Dr. Shuling Yan). 


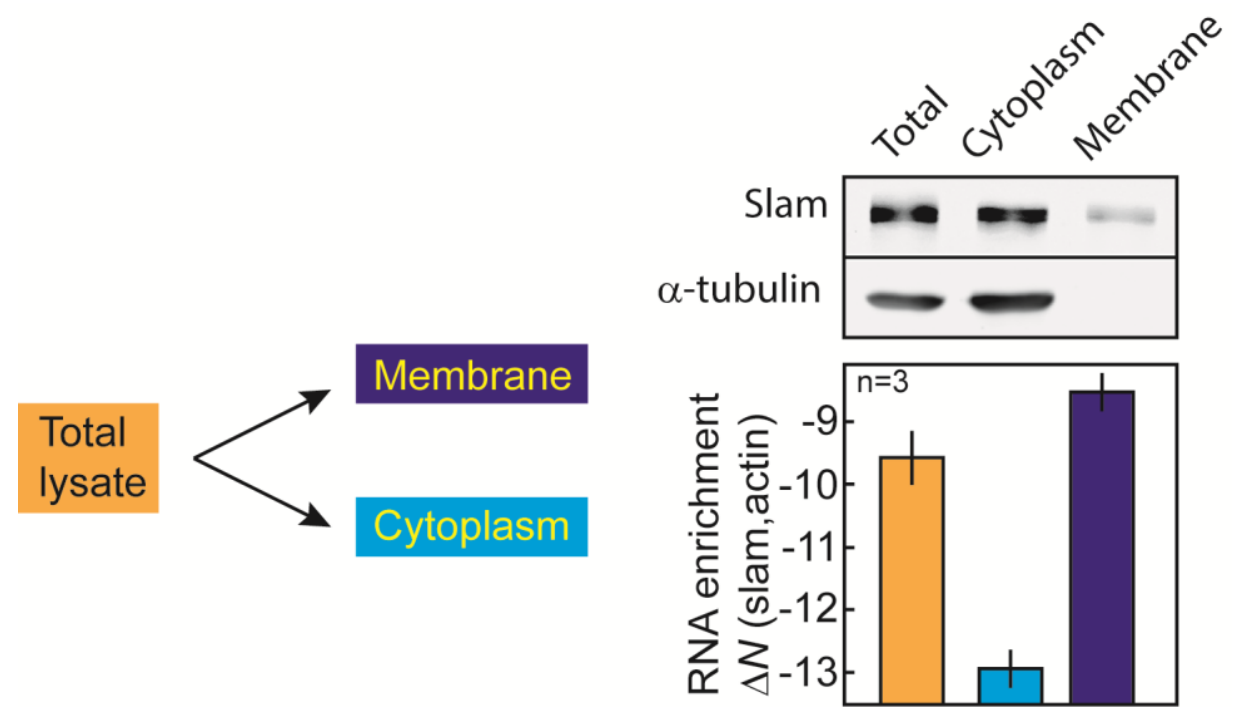

Fig 3.33 slam mRNA is enriched at the membrane. Fractionation of embryos was followed by western blot to detect the protein (with $\alpha$-tubulin as a control) and qRT-PCR (with actin as a control) to detect the mRNA. $n$ refers to the number of independent experiments.

Table 3.2 qRT-PCR threshold cycle $\left(C_{t}\right)$ numbers for three independent fractionation experiments

\begin{tabular}{|l|c|c|c|c|c|c|}
\hline & \multicolumn{2}{|c|}{ Experiment 1 } & \multicolumn{2}{c|}{ Experiment 2 } & \multicolumn{2}{c|}{ Experiment 3 } \\
\hline & actin & slam & actin & slam & actin & slam \\
\hline Total (n) & 16.42 & 25.76 & 14.00 & 23.27 & 14.16 & 24.28 \\
\hline Cytoplasm (n) & 16.88 & 29.44 & 15.89 & 28.87 & 15.91 & 29.31 \\
\hline Membrane (n) & 18.41 & 26.46 & 18.94 & 27.53 & 17.31 & 26.16 \\
\hline M/C Ratio & 0.35 & 7.9 & 0.12 & 2.48 & 0.38 & 8.7 \\
\hline
\end{tabular}

The changes in slam M/C (Membrane/Cytoplasm) ratios in different experiments are comparable to changes in the respective Actin $\mathrm{M} / \mathrm{C}$ ratios.

Next we wondered whether the RNP complex is maintained in a situation where the protein is mistargeted. To test this, we carried out in-situ hybridisation for slam mRNA, followed by immunostaining for Slam protein in nuf embryos. We observed embryos displaying severe nuf phenotype where Slam protein was mislocalised to the cortex and to the lateral membrane and noticed that the mRNA and protein retained 
their colocalisation (Fig 3.34). Thus, we could further confirm that the protein and mRNA are part of the same complex.

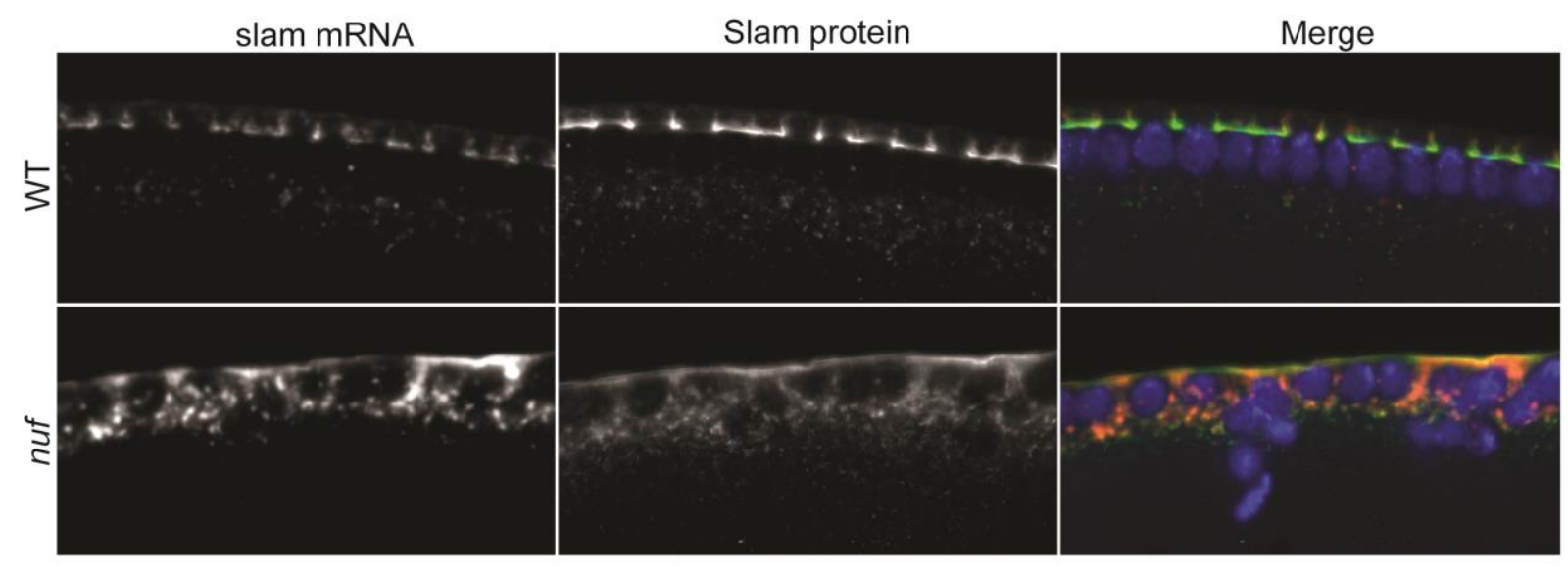

Fig 3.34 slam mRNA and protein also colocalise ectopically. In-situ hybridisation against slam mRNA followed by immunostaining for Slam protein in nuf embryos shows that the complex is retained even when mistargeted.

We wondered whether Slam protein can directly bind its mRNA. In preliminary assays with rabbit reticulocyte lysate in-vitro translation system, it was observed that Slam full-length protein was able to bind to an mRNA localising element on the slam mRNA (Dr. Shuling Yan, unpublished). We carried out electrophoretic mobility-shift assay (EMSA) to determine if Slam protein can directly bind to its localising elements (Fig 3.35).

We used two localising elements of the slam mRNA called 'slam2-1' and 'slam22' which are located in the 5' half of the mRNA. Both the mRNAs had a 5' GFP sequence. The mRNAs were in-vitro transcribed and labelled with Cy3. The protein fragment Slam $\Delta \mathrm{C} 651$ was used, as repeated attempts to purify a soluble recombinant full-length Slam protein were unsuccessful. GST protein and GFP mRNA were used as a negative control. Purification of Slam $\Delta \mathrm{C} 651$ caused another band of a lower molecular weight to co-purify. Analysis of this band using mass spectrometry revealed that this band corresponds to DnaK, a chaperone protein. When we used this preparation for EMSA, it was found that it caused both 'slam2-1' and 'slam2-2' fragments to concentrate into a dark single band from an initial smear. Even the GFP RNA which was initially found as a single band was resolved into two bands at a high Slam $\Delta$ C651 concentration. 
A

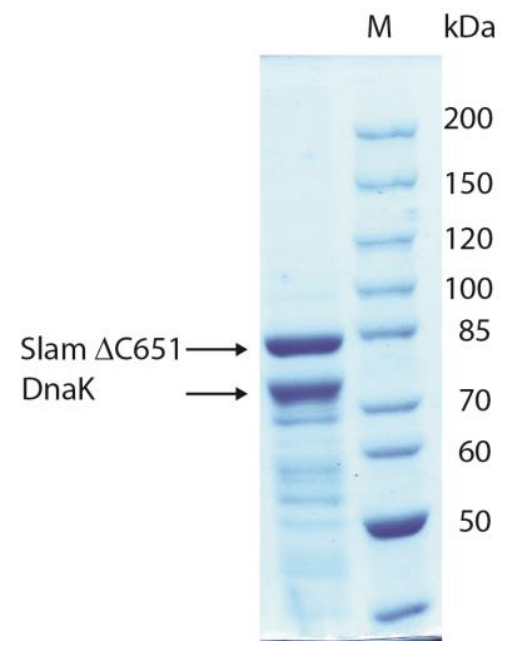

B

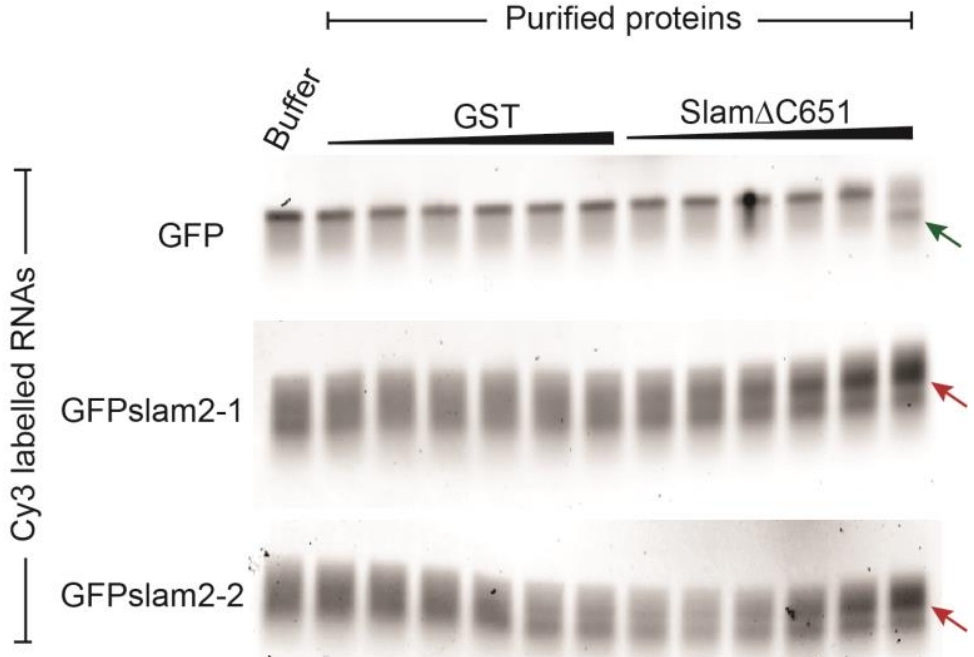

Fig 3.35 Binding assay with Slam $\Delta$ C651 protein and slam mRNA localising elements. (A) Purified Slam $\triangle C 651$ contains a second band corresponding to DnaK (B) EMSA carried out on 1\% Agarose gels. At a higher concentration, Slam $\Delta$ C651 appears to induce/stabilise a second band with the GFP sequence (green arrow) and coalesce the GFPslam2-1 and GFPslam2-2 smears into a prominent band (red arrows).

From the result it appears as if a general 'helicase' activity might be present in the preparation that leads to stabilisation of certain secondary structures of the RNA, which may or may not be due to $\operatorname{Slam} \Delta \mathrm{C} 651$. Further experiments are required to clarify whether Slam protein and the mRNA show a direct binding to each other. 


\section{DISCUSSION}

\subsection{Centrosomes specify the site of cleavage furrow and restrict Slam to the furrow canal}

The first step in cellularisation is the definition of the site of a cleavage furrow. The signals that are required for furrow-specification are as yet unclear. In this study, we were able to confirm previous reports and further define that centrosomes are the source of the initial signal for furrow specification. We found that ectopic centrosomes are able to specify a furrow around themselves even in the absence of nuclei. The involvement of nuclei in furrow specification was ruled out by following SAS6GFP and GFPslam live from cycle 13. It was found that the ectopic centrosomes were a result of additional replication and not nuclear fallout. It was observed that even single centrosomes were able to specify a furrow. However, it was seen that after the initial specification of furrow, GFPslam was not maintained at the furrow in a restricted manner. This can be explained by the fact that the ectopic centrosomes might be only partially functional. This is quite likely since it has been shown that despite having the ability to organise microtubules these ectopic structures lack centrioles (RodriguesMartins et al., 2007). Another possibility is that in the absence of the nuclei, there is a premature basal closure. However, this possibility is unlikely because in wild-type embryos, basal closure does not occur as soon as the membrane invagination front is past the nuclei. The membrane invaginates several micrometres further before expanding laterally. Also, in bnk mutants premature basal closure is observed despite the presence of the nuclei. It suggests that basal closure is a regulated process that takes place independent of the nuclei and the maintenance of the specified furrow is a centrosome-associated function.

We carried out ablation of regular centrosomes in the embryo and were able to identify that Slam restriction to the prospective furrow canal is dependent on the initial signal originating from the centrosomes. Although we were not able to completely ablate the centrosomes, functional ablation could be achieved as the centrosomes lost the ability to specify the furrow. GFPslam was found dispersed and was not maintained at the furrow. Even though it is likely that the ablation resulted in a nuclear-fallout, it does not obscure our interpretation because we have shown that centrosomes are able to specify the furrow in the absence of nuclei. Therefore we conclude that even if ablation caused nuclear-fallout, the resultant effect on furrow-specification was in fact due to ablation of centrosome function. 
The signals deposited by the centrosomes at the prospective site of cleavage furrow are not understood yet. They could be factors needed for vesicle targeting such as exocyst components and/or for vesicle fusion such as SNARE proteins. It has been shown that the recruitment of exocyst complex to the midbody in mammalian cells is mediated by the microtubule motor protein MKLP (Mitotic Kinesin-like protein) and the centrosomal protein centriolin (Gromley et al., 2005). Centrosomal proteins could play similar roles during cleavage furrow specification during Drosophila cellularisation. These vesicle-tethering factors at the plasma membrane could then act as target sites for recycling endosome-derived vesicles.

\subsection{Slam restriction at the furrow canal is dependent upon the recycling endosome}

We aimed to further determine the mechanism through which Slam accumulation and maintenance at the furrow canal takes place. In the absence of evidence for microtubule-based transport of Slam, we looked into the role of vesicular transport in Slam accumulation. When vesicular transport was perturbed by induction of shi phenotype at the onset of cellularisation, we discovered that the behaviour of Slam at the 'old' furrows (metaphase furrows) and 'new' furrows (cellularisation cleavage furrows) was different. While the localisation of Slam at the 'old' furrows was unaffected, it was either not deposited at the 'new' furrows, or was weakly deposited followed by a rapid loss. The loss of Slam preceded loss of membranes as Dlg localisation to the lateral membrane was intact. This differential behaviour of Slam at the 'old' and 'new' border suggests the existence of two different mechanisms for the accumulation of Slam at the prospective furrow canal - one that utilises existing signals from the previous cell cycle while the other that requires fresh signals for the de novo accumulation of Slam.

Since shi inhibits all clathrin-coated vesicular budding, we further investigated the role of specific vesicular trafficking pathways for their role in Slam accumulation at the prospective furrow. Slam is necessary and sufficient for RhoGEF2 localisation at the furrow (Wenzl et al., 2010) and since RhoGEF2 had been shown to be dependent on the recycling endosome function for its accumulation at the furrow (Cao et al., 2008), we looked into the possible role of recycling endosome in Slam localisation. Liveimaging and immunostaing in fixed samples both proved that Slam was not transported on Rab11 positive vesicles. Therefore, we looked for a functional dependence of Slam 
localisation on recycling endosome. We observed that restriction of Slam to the furrow canal was significantly delayed and/or impaired in nuf embryos. Interfering with Rab11 function by injection of a dominant negative Rab11 protein into embryos also caused less efficient restriction of Slam at the furrows. Additionally in nuf mutants, Slam was also found to be mistargeted to the lateral membrane and the cortex. This indicates that restriction of Slam to the basal domain of the invaginating furrow is dependent on the recycling endosome. Since Slam is not transported on recycling endosome vesicles, we concluded that a receptor for Slam must be deposited via the recycling endosome and Slam is subsequently recruited to the membrane through the receptor.

Mislocalisation of Slam to the lateral or apical domains was, however, not noticed in shi mutants. This could be due to the different roles of Shibire and Nuf proteins in the vesicular trafficking pathways. Shi promotes vesicular budding and therefore in shimutants, it is likely that Slam receptor does not reach the site. However, Nuf/Rab11 complex is involved in targeting of the vesicles (Wilson et al., 2005) and therefore, nuf mutants probably are unable to restrict the Slam receptor to the basal domain of both 'old' and 'new' furrows, thus resulting in mislocalisation of Slam that is unbiased between 'old' and 'new' furrows.

A minimal distance is necessary between the two centrosomes to be able to induce a furrow between them (Rappaport, 1986). Due to nuclear fallout, we observed an increased distance between the two centrosome pairs above each nucleus during the interphase of cycle 14 and occasionally noticed an extra furrow formed between them. This observation further confirms that centrosomes are the source of the initial furrow-specifying signals. We showed that singular centrosomes are also able to specify a furrow, which suggests that the increased distance between centrosomes of a pair in nuf mutants seems to cause the individual centrosome to act as an independent furrow-specifying unit.

The roles of centrosomes and the recycling endosome are not mutually exclusive. It has been shown that centrosome regulates recycling endosome organisation and function through its role in regulation of centriolar Rab11 localisation (Hehnly et al., 2012). During syncytial divisions in the early Drosophila embryo, Rab11 maintains a reasonably constant localisation at the MTOC whereas Nuf is recruited to the MTOC only during the furrow invagination phases of cell cycle (Riggs et al., 2007). 
A

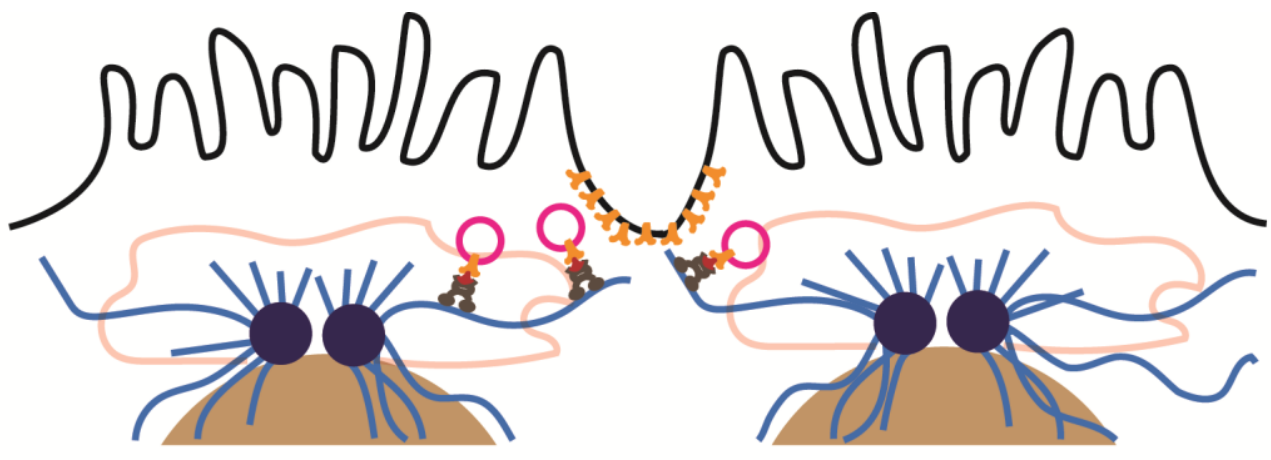

B
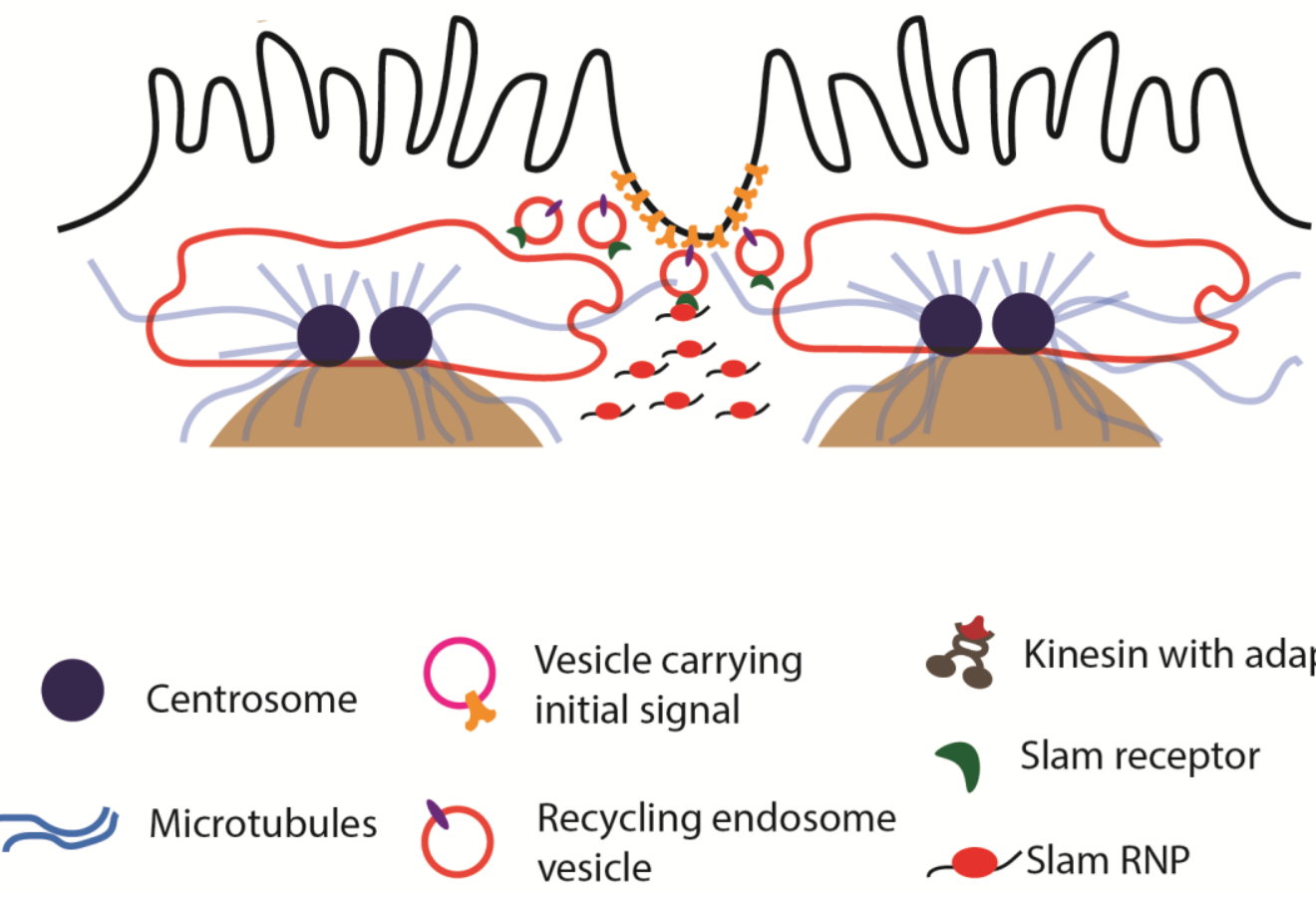

Kinesin with adaptor

$\checkmark$ Slam receptor

Slam RNP

Fig 4.1 Model for the role of centrosomes in targeting of recycling endosome vesicles to the site of cleavage furrow. (A) Centrosomes relay the initial signals to the cleavage furrow site at the region of overlapping asters probably through microtubule-dependent transport (B) Recycling endosome vesicles are targeted to the site of cleavage furrow via the initial cues at the site and deposit a receptor for Slam to which the Slam RNP is recruited from the cytoplasm

Both Rab11 and Nuf localisation has been shown to be dependent on microtubules organised by the centrosomes (Riggs et al., 2007). Therefore, it is conceivable that the mechanism through which centrosomes relay the furrow-specifying signals involves the recycling endosome. Indeed, both in case of centrosome ablation and in nuf embryos we notice lack of restriction of Slam at the furrow canal. Centrosomal proteins such as CNN and CEN disrupt the organisation of pericentriolar material and analysis 
of mutant alleles of their genes have shown that they are needed for cleavage furrow formation even though the microtubular organisation is undisturbed in their mutants (Kao and Megraw, 2009 and references therein). It is plausible that centrosomal proteins contribute to the proper organisation and function of the pericentriolar recycling endosome and consequently exert their effect on the furrows via a microtubule-independent pathway. Further studies are required to clarify the relationship between centrosomal proteins and the recycling endosome.

\subsection{Role of Slam during cellularisation}

Previous work had found that slam is a zygotic gene that has a role in acceleration of membrane invagination (Lecuit et al., 2002). In our lab, it was shown that slam is not just necessary for acceleration of membrane invagination but that it is essential. However, even in the absence of Slam, furrow specification takes place, as demonstrated by the localisation of furrow markers such as Nullo, Diaphanous and Factin (Acharya et al., 2014; Dr. Philip Laupsien, PhD dissertation). This suggests that slam is either not involved in the specification of the site of membrane invagination or that it acts redundantly to other factors. One of the likely candidates for a redundant factor is the zygotic gene nullo, which has been shown to act redundantly to RhoGEF2 (Grosshans et al., 2005). Embryos maternally and zygotically deficient for slam and zygotically deficient for nullo displayed a severely disrupted furrow, as marked by Dia staining. Dia accumulations might either indicate a tendency to aggregate in the absence of a furrow determinant or it might indicate the presence of a regressed furrow. In the latter case it could mean that there is a third independent pathway that controls the furrow specification. In any case however, we could show that nullo and slam act redundant to each other to specify the furrow, even if they might not be the only factors responsible. Therefore, at least one of the functions of Slam during cellularisation is to specify the furrow together with Nullo, via regulation of F-actin at the furrow.

It is unknown how Slam mediates membrane invagination. We tried to uncover a possible mechanism via the actin nucleator Spire which is maternally provided to the embryo. Slam and Spire were shown to interact in a yeast two-hybrid screen (Hybrigenics services; Dr. Philip Laupsien, PhD dissertation). Spire, like formins such as Dia, nucleates unbranched actin filaments though with a different mechanism 
(Quinlan et al., 2005). Dia plays an essential role in cellularisation (Afshar et al., 2000) and therefore we looked into the role of Spire in furrow specification/invagination. spir transheterozygote embryos, however, showed normal cellularisation. Even though some alleles of spir showed a cellularisation defect, we could not establish any link between slam and spir and believe that Spire either has no significant role to play during cellularisation or acts redundantly to other actin nucleators.

We aimed to pulldown the Slam RNP complex in order to obtain more interactors that could reveal the function of Slam. For this purpose we used two approaches immunoprecipitation and GFPtrap. Both methods have their pros and cons. Immunoprecipitation shows higher background than GFPtrap, but tagging slam with an N-terminal GFP causes partial impairment in Slam functionality which might result in loss of certain Slam interactors, especially in the fast phase of cellularisation. It would be best to employ both techniques in the future for pulldown and subsequent Mass spectrometry analysis.

\subsection{Mobility of Slam at the furrow canal}

We confirmed previous reports that Slam is present in both cytoplasmic and membrane fractions in the embryo and were further able to show that it can be dissociated from the membrane using high salt treatment. FRAP experiments have shown that Slam is highly mobile at the onset of cellularisation but is quite stably associated with the membrane during cycle 13 and cycle 14 interphases (Acharya et al., 2014; Dr. Philip Laupsien, PhD dissertation). Slam undergoes a switch-like change from a low mobility to high mobility and vice versa as the embryo undergoes transition from cycle 13 to the onset of cellularisation and moves on to cycle 14 interphase.

The recovery of GFPslam fluorescence at any stage could be due to either of the following mechanisms - a) lateral diffusion of Slam from the unbleached plasma membrane $b$ ) exchange of bleached molecules at the furrow canal with unbleached molecules from the cytoplasm c) active transport of slam receptor via vesicles leading to Slam accumulation d) localised translation at the furrow canal. Lateral diffusion was ruled out as we didn't notice significant reduction in the diameter of the bleached area. GFPslam FRAP in shi mutant background revealed that the recovery of the 'old' borders was not dependent on vesicular transport. This is not surprising as GFPslam accumulation at the 'old' borders was shown to be unaffected in shi mutants compared 
to the 'new' borders. Since 'new' borders could not be analysed in shi mutants, we carried out FRAP in nuf mutants which shows unbiased mislocalisation of Slam. The first striking observation was the constant accumulation of Slam in the region outside the bleached area in a fraction of nuf embryos. This was likely due to the lack of spacial restriction of Slam. Apart from that, a slight increase in mobility was noticed. This suggests that the recycling endosome might be involved in stabilising Slam mobility after the onset of cellularisation.

In the absence of a polarised membrane, Slam seems to consist of a general membrane-affinity as Slam expressed in S2 cells enriches at the cortex (Wenzl et al., 2010). Even in syncytial cycles, expression of GFPslam shows that it associates in a pseudohexagonal array showing that the receptor is maternally provided. It is unlikely that Slam is able to bind directly to the membrane without a receptor because in shi mutants Slam fails to associate to the membrane (apical, lateral or basal) possibly due to the failure of receptor transport. This suggests that Slam receptor might be a general membrane-bound protein that is expressed in varied cell types.

It is conceivable that at the onset of cellularisation when membrane is not polarised, Slam receptor/Slam is generally associated with the membrane and is highly mobile. As cellularisation progresses, the spacial restriction of the Slam receptor by the recycling endosome to the prospective basal domain leads to Slam stability. It is known that the basal domain of the invaginating membrane becomes more stable as cellularisation progresses, probably due to F-actin-mediated prevention of endocytic events (Sokac and Wieschaus, 2008a). It is likely that the basal domain is more stable than the apical or the lateral membrane. Therefore in nuf mutants, it could be that the population of Slam that is mistargeted to the apical and the lateral membrane is the more mobile population. This would also mean that when the shi phenotype was induced at the onset of cellularisation, Slam at the 'old' borders was already restricted to the basal domain, which is why Slam does not show mislocalisation to the apical or lateral domain, nor does it show any change in mobility. Indeed the high mobility phase of Slam lasts very shortly at the onset of cellularisation and possibly the rapid stabilisation of Slam to the basal domain is one reason for its switch to low-mobility. However, this mobility-switch behaviour is not seen in other basal domain markers such as Amphiphysin or PDZ domain of RhoGEF2, which retain high mobility at all stages (Acharya et al., 2014; Dr. Philip Laupsien, PhD dissertation). Therefore, restriction of Slam to the basal domain cannot be accounted as the only reason for its 
unique behaviour. It is not known what additionally determines this switch in the mobility of Slam. Temporal posttranslational modifications affecting Slam conformation and mobility could be worth exploring.

A

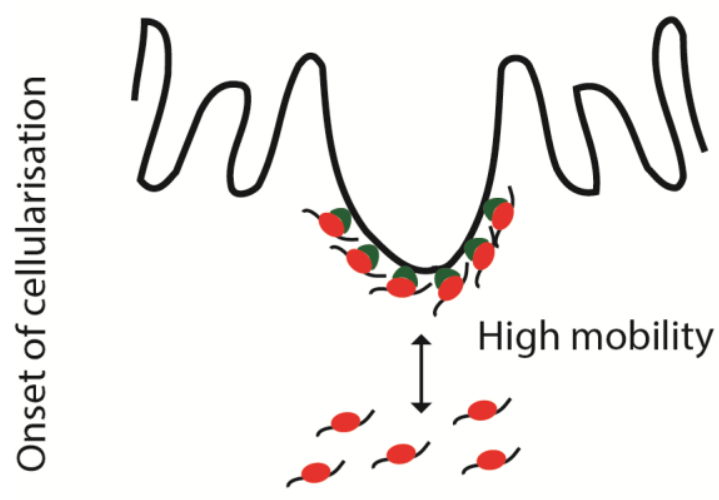

B

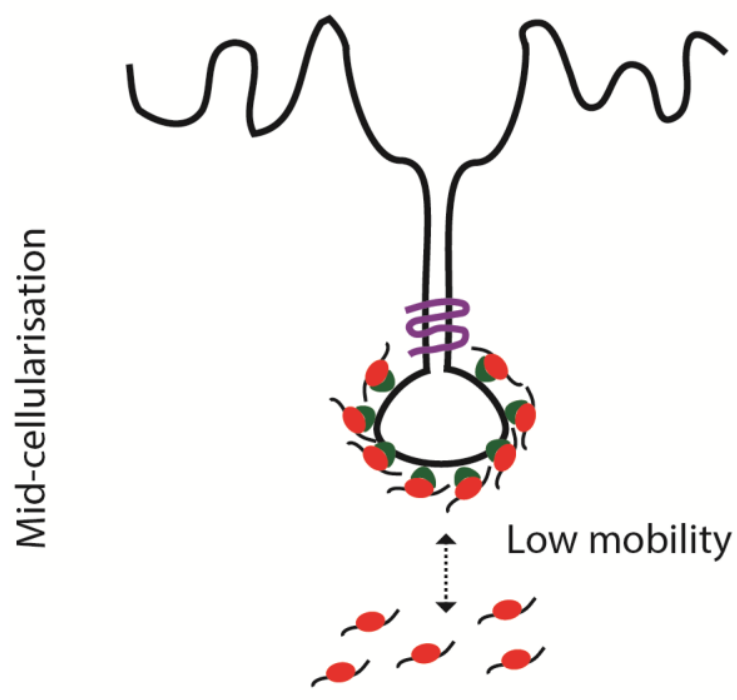

C nuf mutants

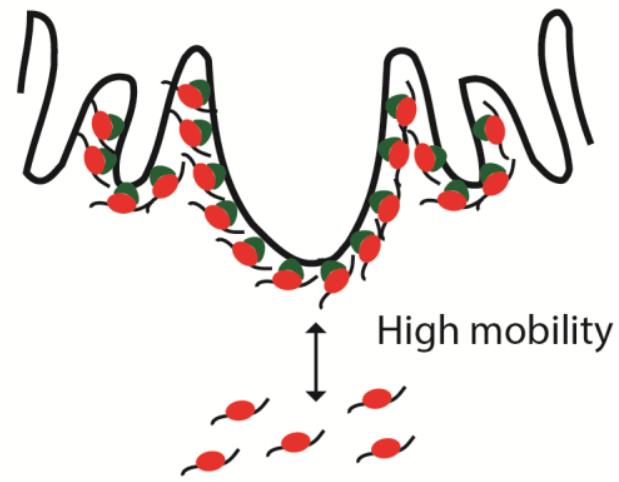

D

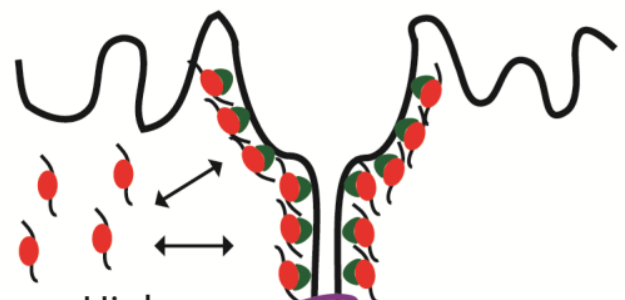

High mobility
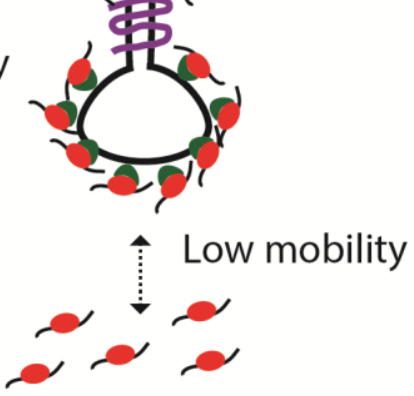

Fig 4.2 Mobility of Slam and the role of recycling endosome in its restriction (A) Slam is highly mobile at the onset of cellularisation (B) During mid-cellularisation, mobility of Slam switches to a lessmobile state $(C)$ In nuf mutants, Slam is mistargeted and fails to be restricted to the prospective basal domain (D) During mid-cellularisation in nuf embryos, the population of Slam associated with the apical and lateral domains of the membrane possibly display higher mobility.

The possibility of localised translation was also explored by injection of cycloheximide into GFPslam embryos at mid-cellularisation followed by FRAP. Slam 
mRNA and protein colocalise with each other at the furrow canal (Wenzl et al., 2010) and therefore it is a plausible mechanism through which Slam localisation is regulated. There was no significant change in the mobility of Slam when new translation was blocked in the embryo during mid-cellularisation. This shows that the (low) mobility of Slam at this stage is due to association-dissociation kinetics and not due to active translation at the furrow canal (or in the entire embryo). The half-life time of Slam was found to be about 42 min which is about two-thirds of the time that cellularisation lasts. Therefore, it is quite likely that new translation of Slam does not play a significant role during mid-cellularisation. However, we could not rule out the role of local translation in the high-mobility phase of Slam at the onset of cellularisation because injection of cycloheximide at an early stage leads to a cell-cycle block. A more efficient photoconvertible tag than the Eos tag could help determine the role of local translation of Slam at the onset of cellularisation.

\subsection{Slam protein and mRNA form a complex}

As mentioned earlier, Slam protein and mRNA colocalise with each other at the furrow canal and basal particles (Wenzl et al., 2010) and have been shown to be part of a complex (Dr. Shuling Yan, unpublished). The mRNA and protein are essential for each other's localisation and function at the furrow canal (Dr. Shuling Yan, unpublished). The protein and the mRNA colocalise even at ectopic regions in nuf embryos further confirming that the complex is a unit. Fractionation of embryos followed by detection of protein and the mRNA revealed that a large part of slam mRNA was enriched at the membrane while about a fifth of the protein population was associated with the membrane.

The association of slam mRNA with its protein raises the likelihood that it carries out some non-coding function at the furrow canal. It could be involved in a) local translation b) local protection c) anchoring d) function. As mentioned earlier, it remains to be seen whether it is involved in local translation at the onset of cellularisation. The possibility that it protects Slam protein from degradation at the furrow canal is ruled out by the fact that a large proportion of Slam is cytoplasmic. The third possibility that it has a role in anchoring Slam protein to the furrow canal could be tested by co-injecting an mRNA that cannot localise but makes a functional Slam protein (mRNA with alternative codons for Slam) and an mRNA that can localise but cannot make a 
functional Slam protein (mRNA with a frame-shift) into a Slam mutant embryo to see whether the mRNA can anchor the protein to the furrow canal. Targeting Slam protein to the furrow canal independent of the mRNA would reveal whether the mRNA is required for the function of Slam. Uncovering the non-coding function of the mRNA would be very interesting as it could lead to a better understanding of the role of localised RNAs in mediating cleavage furrow specification and polarised membrane invagination.

Two redundant localisation elements in the coding region of the mRNA were found in mapping experiments (Dr. Shuling Yan, unpublished). Preliminary results indicated that at least one of them was able to be bound by Slam full-length protein directly in an in-vitro translation system (Dr. Shuling Yan, unpublished). In an attempt to map the region of the protein that is likely to bind to the mRNA, we used a fragment consisting of the first 545 amino acids (Slam $\Delta$ C651) to test for in-vitro binding potential to its mRNA localising elements. EMSA results were however, inconclusive. The Slam protein preparation seemed to contain some kind of general RNA secondary-structure altering/stabilising property, which can either be attributed to an intrinsic property of the protein or due to impurities in the preparation. Further experiments with purer preparations are required to map the RNA-binding region of the protein. Since Slam protein has no known functional domains, this could reveal a novel RNA-binding domain. 


\section{REFERENCES}

Acharya, S., Laupsien, P., Wenzl, C., Yan, S., and Großhans, J. (2014). Function and dynamics of slam in furrow formation in early Drosophila embryo. Dev. Biol. 386, 371-384.

Adam, J.C., Pringle, J.R., and Peifer, M. (2000). Evidence for functional differentiation among Drosophila septins in cytokinesis and cellularization. Mol. Biol. Cell 11, 3123-3135.

Afshar, K., Stuart, B., and Wasserman, S.A. (2000). Functional analysis of the Drosophila diaphanous FH protein in early embryonic development. Dev. Camb. Engl. 127, 1887-1897.

Allen, W.E., Jones, G.E., Pollard, J.W., and Ridley, A.J. (1997). Rho, Rac and Cdc42 regulate actin organization and cell adhesion in macrophages. J. Cell Sci. 110 ( Pt 6), 707-720.

Balasubramanian, M.K., Helfman, D.M., and Hemmingsen, S.M. (1992). A new tropomyosin essential for cytokinesis in the fission yeast S. pombe. Nature 360, 8487.

Barrett, K., Leptin, M., and Settleman, J. (1997). The Rho GTPase and a putative RhoGEF mediate a signaling pathway for the cell shape changes in Drosophila gastrulation. Cell 91, 905-915.

Baruni, J.K., Munro, E.M., and von Dassow, G. (2008). Cytokinetic furrowing in toroidal, binucleate and anucleate cells in C. elegans embryos. J. Cell Sci. 121, 306316.

Bear, J.E., Svitkina, T.M., Krause, M., Schafer, D.A., Loureiro, J.J., Strasser, G.A., Maly, I.V., Chaga, O.Y., Cooper, J.A., Borisy, G.G., et al. (2002). Antagonism between Ena/VASP proteins and actin filament capping regulates fibroblast motility. Cell 109, 509-521.

Bi, E., Maddox, P., Lew, D.J., Salmon, E.D., McMillan, J.N., Yeh, E., and Pringle, J.R. (1998). Involvement of an actomyosin contractile ring in Saccharomyces cerevisiae cytokinesis. J. Cell Biol. 142, 1301-1312.

Bringmann, H., and Hyman, A.A. (2005). A cytokinesis furrow is positioned by two consecutive signals. Nature 436, 731-734.

Cao, J., Albertson, R., Riggs, B., Field, C.M., and Sullivan, W. (2008). Nuf, a Rab11 effector, maintains cytokinetic furrow integrity by promoting local actin polymerization. J. Cell Biol. 182, 301-313.

Chesarone, M.A., DuPage, A.G., and Goode, B.L. (2010). Unleashing formins to remodel the actin and microtubule cytoskeletons. Nat. Rev. Mol. Cell Biol. 11, 62-74.

Daumke, O., Roux, A., and Haucke, V. (2014). BAR domain scaffolds in dynaminmediated membrane fission. Cell 156, 882-892. 
Edgar, B.A., and Schubiger, G. (1986). Parameters controlling transcriptional activation during early Drosophila development. Cell 44, 871-877.

Van Etten, R.A. (1999). Cycling, stressed-out and nervous: cellular functions of c-Abl. Trends Cell Biol. 9, 179-186.

Field, C.M., Coughlin, M., Doberstein, S., Marty, T., and Sullivan, W. (2005). Characterization of anillin mutants reveals essential roles in septin localization and plasma membrane integrity. Dev. Camb. Engl. 132, 2849-2860.

Figard, L., and Sokac, A.M. (2014). A membrane reservoir at the cell surface: unfolding the plasma membrane to fuel cell shape change. Bioarchitecture 4, 39-46.

Figard, L., Xu, H., Garcia, H.G., Golding, I., and Sokac, A.M. (2013). The plasma membrane flattens out to fuel cell-surface growth during Drosophila cellularization. Dev. Cell 27, 648-655.

Finger, F.P., Hughes, T.E., and Novick, P. (1998). Sec3p is a spatial landmark for polarized secretion in budding yeast. Cell $92,559-571$.

Foe, V.E., and Alberts, B.M. (1983). Studies of nuclear and cytoplasmic behaviour during the five mitotic cycles that precede gastrulation in Drosophila embryogenesis. J. Cell Sci. 61, 31-70.

Foe, V.E., Odell, G.M., and Edgar, B.A. (1993). Mitosis and morphogenesis in the Drosophila embryo: Point and counterpoint. Dev. Drosoph. Melanogaster 149-300.

Fox, D.T., and Peifer, M. (2007). Abelson kinase (Abl) and RhoGEF2 regulate actin organization during cell constriction in Drosophila. Development 134, 567-578.

Fujiwara, K., and Pollard, T.D. (1976). Fluorescent antibody localization of myosin in the cytoplasm, cleavage furrow, and mitotic spindle of human cells. J. Cell Biol. 71, 848-875.

Fullilove, S.L., and Jacobson, A.G. (1971). Nuclear elongation and cytokinesis in Drosophila montana. Dev. Biol. 26, 560-577.

Grevengoed, E.E., Fox, D.T., Gates, J., and Peifer, M. (2003). Balancing different types of actin polymerization at distinct sites: roles for Abelson kinase and Enabled. J. Cell Biol. 163, 1267-1279.

Gromley, A., Yeaman, C., Rosa, J., Redick, S., Chen, C.-T., Mirabelle, S., Guha, M., Sillibourne, J., and Doxsey, S.J. (2005). Centriolin anchoring of exocyst and SNARE complexes at the midbody is required for secretory-vesicle-mediated abscission. Cell 123, 75-87.

Grosshans, J., Wenzl, C., Herz, H.-M., Bartoszewski, S., Schnorrer, F., Vogt, N., Schwarz, H., and Müller, H.-A. (2005). RhoGEF2 and the formin Dia control the formation of the furrow canal by directed actin assembly during Drosophila cellularisation. Dev. Camb. Engl. 132, 1009-1020.

Hain, D., Langlands, A., Sonnenberg, H.C., Bailey, C., Bullock, S.L., and Müller, H.-A.J. (2014). The Drosophila MAST kinase Drop out is required to initiate membrane 
compartmentalisation during cellularisation and regulates dynein-based transport. Dev. Camb. Engl. 141, 2119-2130.

Hehnly, H., Chen, C.-T., Powers, C.M., Liu, H.-L., and Doxsey, S. (2012). The centrosome regulates the Rab11- dependent recycling endosome pathway at appendages of the mother centriole. Curr. Biol. CB 22, 1944-1950.

Hickson, G.R.X., Matheson, J., Riggs, B., Maier, V.H., Fielding, A.B., Prekeris, R., Sullivan, W., Barr, F.A., and Gould, G.W. (2003). Arfophilins are dual Arf/Rab 11 binding proteins that regulate recycling endosome distribution and are related to Drosophila nuclear fallout. Mol. Biol. Cell 14, 2908-2920.

Hinshaw, J.E., and Schmid, S.L. (1995). Dynamin self-assembles into rings suggesting a mechanism for coated vesicle budding. Nature 374, 190-192.

Horgan, C.P., Oleksy, A., Zhdanov, A.V., Lall, P.Y., White, I.J., Khan, A.R., Futter, C.E., McCaffrey, J.G., and McCaffrey, M.W. (2007). Rab11-FIP3 is critical for the structural integrity of the endosomal recycling compartment. Traffic Cph. Den. 8, 414430.

Hunter, C., and Wieschaus, E. (2000). Regulated expression of nullo is required for the formation of distinct apical and basal adherens junctions in the Drosophila blastoderm. J. Cell Biol. 150, 391-401.

Hunter, C., Sung, P., Schejter, E.D., and Wieschaus, E. (2002). Conserved Domains of the Nullo Protein Required for Cell-Surface Localization and Formation of Adherens Junctions. Mol. Biol. Cell 13, 146-157.

Ishizaki, T., Morishima, Y., Okamoto, M., Furuyashiki, T., Kato, T., and Narumiya, S. (2001). Coordination of microtubules and the actin cytoskeleton by the Rho effector mDia1. Nat. Cell Biol. 3, 8-14.

Kao, L.-R., and Megraw, T.L. (2009). Centrocortin cooperates with centrosomin to organize Drosophila embryonic cleavage furrows. Curr. Biol. CB 19, 937-942.

Knoblich, J.A. (2000). Epithelial polarity: The ins and outs of the fly epidermis. Curr. Biol. 10, R791-R794.

Krause, M., Bear, J.E., Loureiro, J.J., and Gertler, F.B. (2002). The Ena/VASP enigma. J. Cell Sci. 115, 4721-4726.

Lecuit, T., and Wieschaus, E. (2000). Polarized insertion of new membrane from a cytoplasmic reservoir during cleavage of the Drosophila embryo. J. Cell Biol. 150, 849860.

Lecuit, T., Samanta, R., and Wieschaus, E. (2002). slam encodes a developmental regulator of polarized membrane growth during cleavage of the Drosophila embryo. Dev. Cell 2, 425-436.

Lee, D.M., and Harris, T.J.C. (2013). An Arf-GEF regulates antagonism between endocytosis and the cytoskeleton for Drosophila blastoderm development. Curr. Biol. CB 23, 2110-2120. 
Li, K., and Kaufman, T.C. (1996). The homeotic target gene centrosomin encodes an essential centrosomal component. Cell 85, 585-596.

Mabuchi, I., and Okuno, M. (1977). The effect of myosin antibody on the division of starfish blastomeres. J. Cell Biol. 74, 251-263.

Mavrakis, M., Rikhy, R., and Lippincott-Schwartz, J. (2009). Plasma Membrane Polarity and Compartmentalization are Established Before Cellularization in the Fly Embryo. Dev. Cell 16, 93-104.

McCleland, M.L., and O'Farrell, P.H. (2008). RNAi of mitotic cyclins in Drosophila uncouples the nuclear and centrosome cycle. Curr. Biol. CB 18, 245-254.

Megraw, T.L., Li, K., Kao, L.R., and Kaufman, T.C. (1999). The centrosomin protein is required for centrosome assembly and function during cleavage in Drosophila. Dev. Camb. Engl. 126, 2829-2839.

Merrill, P.T., Sweeton, D., and Wieschaus, E. (1988). Requirements for autosomal gene activity during precellular stages of Drosophila melanogaster. Dev. Camb. Engl. 104, 495-509.

Meyer, W.J., Schreiber, S., Guo, Y., Volkmann, T., Welte, M.A., and Müller, H.A.J. (2006). Overlapping functions of argonaute proteins in patterning and morphogenesis of Drosophila embryos. PLoS Genet. 2, e134.

Motegi, F., Velarde, N.V., Piano, F., and Sugimoto, A. (2006). Two phases of astral microtubule activity during cytokinesis in C. elegans embryos. Dev. Cell 10, 509-520.

Murthy, M., Teodoro, R.O., Miller, T.P., and Schwarz, T.L. (2010). Sec5, a member of the exocyst complex, mediates Drosophila embryo cellularization. Dev. Camb. Engl. 137, 2773-2783.

Padash Barmchi, M., Rogers, S., and Häcker, U. (2005). DRhoGEF2 regulates actin organization and contractility in the Drosophila blastoderm embryo. J. Cell Biol. 168, $575-585$.

Palazzo, A.F., Cook, T.A., Alberts, A.S., and Gundersen, G.G. (2001). mDia mediates Rho-regulated formation and orientation of stable microtubules. Nat. Cell Biol. 3, 723-729.

Papoulas, O., Hays, T.S., and Sisson, J.C. (2005). The golgin Lava lamp mediates dynein-based Golgi movements during Drosophila cellularization. Nat. Cell Biol. 7, 612-618.

Peel, N., Stevens, N.R., Basto, R., and Raff, J.W. (2007). Overexpressing centriolereplication proteins in vivo induces centriole overduplication and de novo formation. Curr. Biol. CB 17, 834-843.

Pelissier, A., Chauvin, J.-P., and Lecuit, T. (2003). Trafficking through Rab11 Endosomes Is Required for Cellularization during Drosophila Embryogenesis. Curr. Biol. 13, 1848-1857. 
Postner, M.A., and Wieschaus, E.F. (1994). The nullo protein is a component of the actin-myosin network that mediates cellularization in Drosophila melanogaster embryos. J. Cell Sci. 107 ( Pt 7), 1863-1873.

Quinlan, M.E., Heuser, J.E., Kerkhoff, E., and Mullins, R.D. (2005). Drosophila Spire is an actin nucleation factor. Nature $433,382-388$.

Raff, J.W., and Glover, D.M. (1989). Centrosomes, and not nuclei, initiate pole cell formation in Drosophila embryos. Cell 57, 611-619.

Rappaport, R. (1961). Experiments concerning the cleavage stimulus in sand dollar eggs. J. Exp. Zool. 148, 81-89.

Rappaport, R. (1985). Repeated furrow formation from a single mitotic apparatus in cylindrical sand dollar eggs. J. Exp. Zool. 234, 167-171.

Rappaport, R. (1986). Establishment of the mechanism of cytokinesis in animal cells. Int. Rev. Cytol. 105, 245-281.

Riggs, B., Rothwell, W., Mische, S., Hickson, G.R.X., Matheson, J., Hays, T.S., Gould, G.W., and Sullivan, W. (2003). Actin cytoskeleton remodeling during early Drosophila furrow formation requires recycling endosomal components Nuclear-fallout and Rab11. J. Cell Biol. 163, 143-154.

Riggs, B., Fasulo, B., Royou, A., Mische, S., Cao, J., Hays, T.S., and Sullivan, W. (2007). The concentration of Nuf, a Rab11 effector, at the microtubule-organizing center is cell cycle regulated, dynein-dependent, and coincides with furrow formation. Mol. Biol. Cell 18, 3313-3322.

Robinson, J.T., Wojcik, E.J., Sanders, M.A., McGrail, M., and Hays, T.S. (1999). Cytoplasmic dynein is required for the nuclear attachment and migration of centrosomes during mitosis in Drosophila. J. Cell Biol. 146, 597-608.

Rodrigues-Martins, A., Bettencourt-Dias, M., Riparbelli, M., Ferreira, C., Ferreira, I., Callaini, G., and Glover, D.M. (2007). DSAS-6 organizes a tube-like centriole precursor, and its absence suggests modularity in centriole assembly. Curr. Biol. CB $17,1465-1472$.

Rose, L.S., and Wieschaus, E. (1992). The Drosophila cellularization gene nullo produces a blastoderm-specific transcript whose levels respond to the nucleocytoplasmic ratio. Genes Dev. 6, 1255-1268.

Rothwell, W.F., Zhang, C.X., Zelano, C., Hsieh, T.S., and Sullivan, W. (1999). The Drosophila centrosomal protein Nuf is required for recruiting Dah, a membrane associated protein, to furrows in the early embryo. J. Cell Sci. 112, 2885-2893.

Royou, A., Field, C., Sisson, J.C., Sullivan, W., and Karess, R. (2004). Reassessing the Role and Dynamics of Nonmuscle Myosin II during Furrow Formation in Early Drosophila Embryos. Mol. Biol. Cell 15, 838-850.

Schejter, E.D., and Wieschaus, E. (1993). bottleneck acts as a regulator of the microfilament network governing cellularization of the Drosophila embryo. Cell 75, 373-385. 
Schweisguth, F., Lepesant, J.A., and Vincent, A. (1990). The serendipity alpha gene encodes a membrane-associated protein required for the cellularization of the Drosophila embryo. Genes Dev. 4, 922-931.

Shubeita, G.T., Tran, S.L., Xu, J., Vershinin, M., Cermelli, S., Cotton, S.L., Welte, M.A., and Gross, S.P. (2008). Consequences of motor copy number on the intracellular transport of kinesin-1-driven lipid droplets. Cell 135, 1098-1107.

Simpson, L., and Wieschaus, E. (1990). Zygotic activity of the nullo locus is required to stabilize the actin-myosin network during cellularization in Drosophila. Dev. Camb. Engl. 110, 851-863.

Sisson, J.C., Field, C., Ventura, R., Royou, A., and Sullivan, W. (2000). Lava lamp, a novel peripheral golgi protein, is required for Drosophila melanogaster cellularization. J. Cell Biol. 151, 905-918.

Sokac, A.M., and Wieschaus, E. (2008a). Local actin-dependent endocytosis is zygotically controlled to initiate Drosophila cellularization. Dev. Cell 14, 775-786.

Sokac, A.M., and Wieschaus, E. (2008b). Zygotically controlled F-actin establishes cortical compartments to stabilize furrows during Drosophila cellularization. J. Cell Sci. $121,1815-1824$

Sommi, P., Ananthakrishnan, R., Cheerambathur, D.K., Kwon, M., Morales-Mulia, S., Brust-Mascher, I., and Mogilner, A. (2010). A mitotic kinesin-6, Pav-KLP, mediates interdependent cortical reorganization and spindle dynamics in Drosophila embryos. J. Cell Sci. 123, 1862-1872.

Stein, J.A., Broihier, H.T., Moore, L.A., and Lehmann, R. (2002). Slow as molasses is required for polarized membrane growth and germ cell migration in Drosophila. Dev. Camb. Engl. 129, 3925-3934.

Stevenson, V., Hudson, A., Cooley, L., and Theurkauf, W.E. (2002). Arp2/3Dependent Psuedocleavage Furrow Assembly in Syncytial Drosophila Embryos. Curr. Biol. 12, 705-711.

Sullivan, W., Fogarty, P., and Theurkauf, W. (1993). Mutations affecting the cytoskeletal organization of syncytial Drosophila embryos. Development 118, 12451254.

Swanson, M.M., and Poodry, C.A. (1981). The shibire(ts) mutant of Drosophila: a probe for the study of embryonic development. Dev. Biol. 84, 465-470.

Theurkauf, W.E. (1994). Premature microtubule-dependent cytoplasmic streaming in cappuccino and spire mutant oocytes. Science 265, 2093-2096.

Thomas, J.H., and Wieschaus, E. (2004). src64 and tec29 are required for microfilament contraction during Drosophila cellularization. Dev. Camb. Engl. 131, 863-871. 
Vizcarra, C.L., Kreutz, B., Rodal, A.A., Toms, A.V., Lu, J., Zheng, W., Quinlan, M.E., Eck, M.J. (2011). Structure and function of the interacting domains of Spire and Fmn-family formins. Proc. Natl. Acad. Sci. U. S. A. 108, 11884-11889.

Wen, Y., Eng, C.H., Schmoranzer, J., Cabrera-Poch, N., Morris, E.J.S., Chen, M., Wallar, B.J., Alberts, A.S., and Gundersen, G.G. (2004). EB1 and APC bind to mDia to stabilize microtubules downstream of Rho and promote cell migration. Nat. Cell Biol. $6,820-830$.

Wenzl, C., Yan, S., Laupsien, P., and Grosshans, J. (2010). Localization of RhoGEF2 during Drosophila cellularization is developmentally controlled by Slam. Mech. Dev. 127, 371-384.

Wheatley, S.P., and Wang, Y. (1996). Midzone microtubule bundles are continuously required for cytokinesis in cultured epithelial cells. J. Cell Biol. 135, 981-989.

Wiedenmann, J., Ivanchenko, S., Oswald, F., Schmitt, F., Röcker, C., Salih, A., Spindler, K.-D., and Nienhaus, G.U. (2004). EosFP, a fluorescent marker protein with UV-inducible green-to-red fluorescence conversion. Proc. Natl. Acad. Sci. U. S. A. 101, 15905-15910.

Wieschaus, E., and Sweeton, D. (1988). Requirements for X-linked zygotic gene activity during cellularization of early Drosophila embryos. Dev. Camb. Engl. 104, 483493.

Williams, B.C., Riedy, M.F., Williams, E.V., Gatti, M., and Goldberg, M.L. (1995). The Drosophila kinesin-like protein KLP3A is a midbody component required for central spindle assembly and initiation of cytokinesis. J. Cell Biol. 129, 709-723.

Wilson, G.M., Fielding, A.B., Simon, G.C., Yu, X., Andrews, P.D., Hames, R.S., Frey, A.M., Peden, A.A., Gould, G.W., and Prekeris, R. (2005). The FIP3-Rab11 Protein Complex Regulates Recycling Endosome Targeting to the Cleavage Furrow during Late Cytokinesis. Mol. Biol. Cell 16, 849-860.

Yan, S., Lv, Z., Winterhoff, M., Wenzl, C., Zobel, T., Faix, J., Bogdan, S., and Grosshans, J. (2013). The F-BAR protein Cip4/Toca-1 antagonizes the formin Diaphanous in membrane stabilization and compartmentalization. J. Cell Sci. 126, 1796-1805.

Zalokar, M., and Erk, I. (1976). Division and migration of nuclei during early embryogenesis of Drosophila melanogaster. J. Microbiol. Cell. 25, 97-106. 